\title{
Gold(I)-catalyzed one-pot and diastereoselective synthesis of trans-2-silyl-4,5-dihydrofurans from propargylsilanes and aldehydes
}

Sergio Fernández, Javier Santamaría* and Alfredo Ballesteros*

Departamento de Química Orgánica e Inorgánica and Instituto Universitario de Química Organometálica "Enrique Moles", Unidad Asociada al C.S.I.C. Universidad de Oviedo, C/ Julián Clavería, 8, 33006 Oviedo, Spain

\section{Table of Contents}

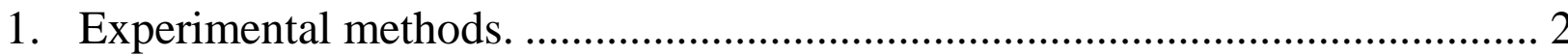

2. Experimental procedure for the synthesis of diastereomeric mixtures of transcis-2-sylil-4,5-dihydrofurans $3 / 4$......................................................................... 3

3. General procedure for the synthesis and isomerization of 2-silyl-4,5dihydrofurans 4 and naphthalene 6 .

4. Experimental procedure for the formation of trans-2-silyl-4,5-dihydrofurans from a cis/trans mixture of isomers or from a syn/anti mixture or corresponding alkynols 5 .

5. Experimental procedure for the synthesis of homopropargyl alcohols syn-5 for Hammett plot and anti-5 for the synthesis of pure trans-dihydrofurans $4 \mathrm{j}, 4 \mathrm{k}$ and $4 \mathrm{p}$.

6. General procedure for the synthesis of cis-2-silyl-4,5-dihydrofurans 3.......... 23

7. NMR Spectra 


\section{Experimental methods.}

All operations were carried out under argon atmosphere using conventional Schlenck techniques. All common reagents were obtained from commercial suppliers. 1,2-Dichloroethane and methanol were distilled from calcium hydride prior to its use. Hexane and ethyl acetate were used from commercial suppliers.

The synthesis of propargylsilanes $\mathbf{1}$ have been accomplished through lithiation of propargyl precursors followed by silyilation. ${ }^{1}$

TLC was performed on aluminium-backed plates coated with silica gel 60, with F254 indicator and flash chromatographic columns were carried out on silica gel (50-200 micron). High-resolution mass spectra were determined on an HPLC-MS/MS QTOF Bruker Impact mass spectrometer. NMR spectra were run on Bruker AV-300 and DPX-300 spectrometers using $\mathrm{CDCl}_{3}$ as solvent. Melting points were measured on a Büchi-Tottoli apparatus and were not corrected.

${ }^{1}$ S. Fernández, J. González, J. Santamaría, A. Ballesteros, Angew. Chem., Int. Ed. 2019, 58, 10703-10707. 


\section{Experimental procedure for the synthesis of diastereomeric mixtures of trans-cis-2-sylil-4,5-dihydrofurans 3/4.}

To a solution $0.24 \mathrm{mmol}$ of propargylsilane 1 in $1 \mathrm{~mL}$ of dry 1,2-dichloroethane at $25^{\circ} \mathrm{C}$ were sequentially added $0.2 \mathrm{mmol}$ of the corresponding aldehyde 2 and $11.2 \mathrm{mg}$ of the gold catalyst (5 mol\%). The reaction was then stirred for $25 \mathrm{~min}$. Upon starting material disappearance, $161 \mu \mathrm{L}$ (128 mg; 4 mmol; 20 equivalents) of dry methyl alcohol were added and the reaction heated, in an oil bath, at $60{ }^{\circ} \mathrm{C}$ for 6 hours. Then, $8.2 \mathrm{mg}(0.02 \mathrm{mmol} ; 10 \mathrm{~mol} \%)$ of $\left(p-\mathrm{MeO}-\mathrm{C}_{6} \mathrm{H}_{4}\right)_{3} \mathrm{P}$ were added for catalyst deactivation. After cooling down, the solvents were removed under vacuum and the residue analyzed by NMR using $\mathrm{CH}_{2} \mathrm{Br}_{2}$ as internal standard. Trans/cis diastereomeric ratios are represented in brackets. As a representative example, a mixture of dihydrofurans $\mathbf{3 b} / \mathbf{4 b}$ was synthesized at 1 mmol scale, using 1,2 mmol of corresponding propargylsilane 1 (362 mg), 1 mmol of $p$-tolualdehyde $2 \mathbf{b}(120 \mathrm{mg}), 56 \mathrm{mg}$ ( $5 \mathrm{~mol} \%)$ of the gold catalyst and $5 \mathrm{~mL}$ of 1,2-dichloroethane. No significant differences were observed following this procedure, as $343 \mathrm{mg}(98 \%)$ of mixture of dihydrofurans 3b/4b were obtained.
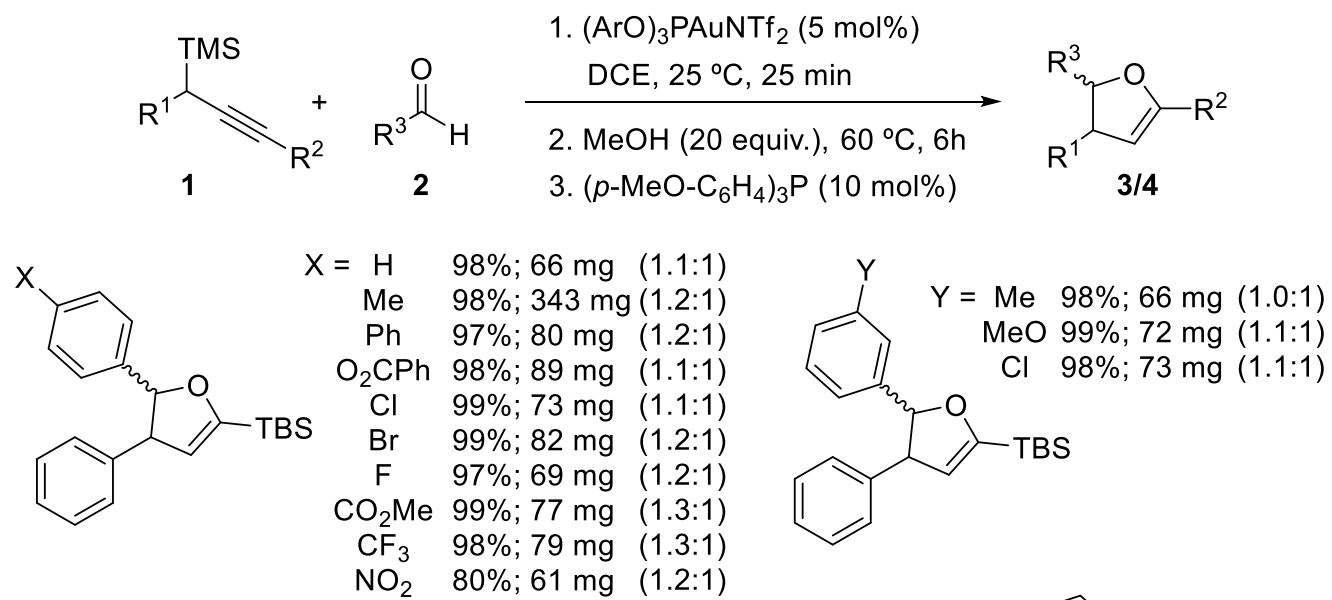<smiles>[Z]c1cccc([X])c1</smiles>

$\mathrm{Z}=\mathrm{Me} 99 \% ; 72 \mathrm{mg}(1.1: 1)$ $\mathrm{Br} 99 \% ; 97 \mathrm{mg}(1.2: 1)$<smiles>CCC[C@H]1OC([135I])=CC1c1ccccc1</smiles><smiles>Cc1ccccc1[C@@H]1OC([13C](C)(C)C)=CC1c1ccccc1</smiles><smiles>CC(C)(C)C1=CC(c2ccccc2)C(c2cccc3ccccc23)O1</smiles><smiles>Cc1ccc(C2C=C([Pb])O[C@@H]2c2ccc(C)cc2)cc1</smiles><smiles></smiles>

$99 \% ; 69 \mathrm{mg}(1.2: 1)$

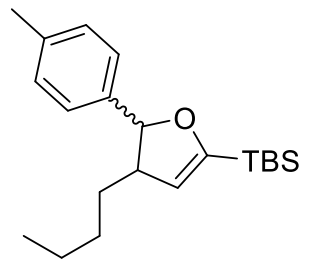

$99 \% ; 65 \mathrm{mg}(1.0: 1)$

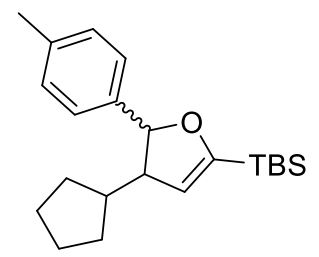

$99 \% ; 68 \mathrm{mg}(1.1: 1)$

TBS $=t$-butyldimethylsilyl; TIPS $=$ triisopropylsilyl $\mathrm{Ar}=\operatorname{tris}(2,6-\mathrm{di}-t$-butylphenyl 


\section{General procedure for the synthesis and isomerization of 2-silyl-4,5- dihydrofurans 4 and naphthalene 6.}<smiles>[R]C#CC([R])[As]</smiles>

1<smiles>[R]C=O</smiles>

2

\author{
1. (ArO) $)_{3} \mathrm{PAuNTf}_{2}(5 \mathrm{~mol} \%)$ \\ DCE, $25^{\circ} \mathrm{C}, 25 \mathrm{~min}$ \\ 2. ${ }^{\mathrm{t}} \mathrm{BuOH}, 60^{\circ} \mathrm{C}, \mathrm{th}$ \\ 3. $\left(p-\mathrm{MeO}-\mathrm{C}_{6} \mathrm{H}_{4}\right)_{3} \mathrm{P}(10 \mathrm{~mol} \%)$
}<smiles>[R]C1=CC([R])C([R])O1</smiles>

4

To a reaction prepared in a similar manner to the mixture of the previous section, $23 \mu \mathrm{L}$ of dry tertbutyl alcohol (18.8 $\mathrm{mg}$; $0.24 \mathrm{mmol}$.) were added instead of methanol and the reaction heated, in an oil bath, at $60{ }^{\circ} \mathrm{C}$ for the period reported in the text. After that period, $8.2 \mathrm{mg}(0.02 \mathrm{mmol} ; 10 \mathrm{~mol} \%)$ of $\left(p-\mathrm{MeO}-\mathrm{C}_{6} \mathrm{H}_{4}\right)_{3} \mathrm{P}$ were added to inactivate the gold catalyst. After cooling down, solvents were removed under vacuum and the residue purified under deactivated aluminium oxide ${ }^{2}$ flash chromatography to obtain the corresponding trans-2-silyl-4,5-dihydrofurans $\mathbf{4}$ or naphthalene $\mathbf{6}$ for 3,5-dimethoxybenzaldehyde $2 \mathbf{w}$.

\section{Experimental procedure for the formation of trans-2-silyl-4,5- dihydrofurans from a cis/trans mixture of isomers or from a syn/anti mixture or corresponding alkynols 5.}

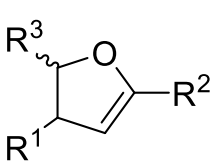

$3 / 4$
1. $(\mathrm{ArO})_{3} \mathrm{PAuNTf}_{2}(5 \mathrm{~mol} \%)$

2. $\left(p-\mathrm{MeO}-\mathrm{C}_{6} \mathrm{H}_{4}\right)_{3} \mathrm{P}(10 \mathrm{~mol} \%)$<smiles>[R]C1=CC([R])[C@@H]([R3])O1</smiles>

4

To a solution of $0.2 \mathrm{mmol}$ of dihydrofurans mixture $3 / 4$ in $1 \mathrm{~mL}$ of dry 1,2-dichloroethane, $11.2 \mathrm{mg}$ of the gold catalyst $\left(5 \mathrm{~mol} \%\right.$ ) were added and the reaction stirred, in an oil bath, at $60{ }^{\circ} \mathrm{C}$ for 6 hours. Then, $8.2 \mathrm{mg}(0.02 \mathrm{mmol} ; 10 \mathrm{~mol} \%)$ of $\left(p-\mathrm{MeO}-\mathrm{C}_{6} \mathrm{H}_{4}\right)_{3} \mathrm{P}$ were added for catalyst deactivation. After cooling down, the solvents were removed under vacuum and the residue purified by chromatographic column through deactivated aluminium oxide yielding pure trans-2-silyl-4,5-dihydrofurans 4.

Identical procedure was followed starting from a mixture of alkynols 5. No significant changes were observed when the reaction was performed at $1 \mathrm{mmol}$ scale. As a representative example, trans-2silyldihydrofuran $\mathbf{4 b}$ was obtained in $99 \%$ yield (346 mg).

\footnotetext{
${ }^{2}$ For the preparation of deactivated aluminium oxide: To a flask containing $460 \mathrm{~g}$ of basic aluminum oxide, $50 \mathrm{~mL}$ of water (1 mL / $9.2 \mathrm{~g})$ were added and it was vigorously shaken to obtain a free-floating solid, ready to be used.
} 


\section{Trans-tert-butyldimethyl-4,5-diphenyl-4,5-dihydrofuran-2-yl)dimethylsilane (4a)}

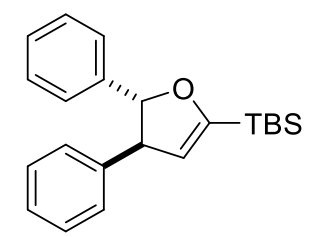

Yield: 99\%, $67 \mathrm{mg}$

Colorless oil

$\mathrm{R}_{\mathrm{f}}\left(\mathrm{SiO}_{2}\right)=0.22($ Hexanes/ Ethyl acetate, (200:1)).

${ }^{1} \mathrm{H}$ NMR $\left(300 \mathrm{MHz}, \mathrm{CDCl}_{3}\right) \delta=7.44-7.23(\mathrm{~m}, 10 \mathrm{H}), 5.35(\mathrm{~d}, J=7.1 \mathrm{~Hz}, 1 \mathrm{H}), 5.31(\mathrm{~d}, J=2.4 \mathrm{~Hz}$, $1 \mathrm{H}), 4.06(\mathrm{dd}, J=7.2$ and $2.5 \mathrm{~Hz}, 1 \mathrm{H}), 1.09(\mathrm{~s}, 9 \mathrm{H}), 0.28(\mathrm{~s}, 3 \mathrm{H}), 0.26(\mathrm{~s}, 3 \mathrm{H})$.

${ }^{13} \mathrm{C} \mathrm{NMR}\left(75 \mathrm{MHz}, \mathrm{CDCl}_{3}\right) \delta=162.0(\mathrm{C}), 144.5(\mathrm{C}), 143.6(\mathrm{C}), 128.8(2 \times \mathrm{CH}), 128.6(2 \times \mathrm{CH})$, $127.8(2 \times \mathrm{CH}), 127.6(\mathrm{CH}), 126.9(\mathrm{CH}), 125.4(2 \times \mathrm{CH}), 116.3(\mathrm{CH}), 92.1(\mathrm{CH}), 59.6(\mathrm{CH}), 26.8(3$ x $\left.\mathrm{CH}_{3}\right), 16.7(\mathrm{C}),-6.2\left(\mathrm{CH}_{3}\right),-6.3\left(\mathrm{CH}_{3}\right)$.

HRMS (ESI) m/z: [M + H] ${ }^{+}$Calcd. for $\mathrm{C}_{22} \mathrm{H}_{29} \mathrm{OSi}$ : 337.2139; Found: 337.2127.

\section{Trans-tert-butyldimethyl(4-phenyl-5-(p-tolyl)-4,5-dihydrofuran-2-yl)silane (4b)}

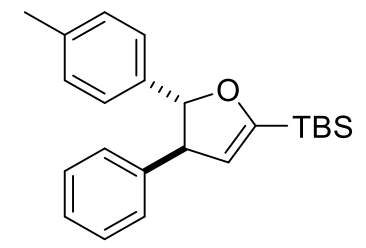

Yield: $99 \%, 70 \mathrm{mg}$

Colorless oil

$\mathrm{R}_{\mathrm{f}}\left(\mathrm{SiO}_{2}\right)=0.24$ (Hexanes/ Ethyl acetate, $\left.(200: 1)\right)$.

${ }^{1} \mathrm{H}$ NMR $\left(300 \mathrm{MHz}, \mathrm{CDCl}_{3}\right) \delta=7.39-7.31(\mathrm{~m}, 2 \mathrm{H}), 7.30-7.13(\mathrm{~m}, 7 \mathrm{H}), 5.28(\mathrm{~d}, J=1.1 \mathrm{~Hz}, 1 \mathrm{H}), 5.26$ $(\mathrm{d}, J=3.5 \mathrm{~Hz}, 1 \mathrm{H}), 4.01(\mathrm{dd}, J=7.2,2.4 \mathrm{~Hz}, 1 \mathrm{H}), 2.36(\mathrm{~s}, 3 \mathrm{H}), 1.04(\mathrm{~s}, 9 \mathrm{H}), 0.23(\mathrm{~s}, 3 \mathrm{H}), 0.21$ (s, $3 \mathrm{H})$.

${ }^{13} \mathrm{C} \mathrm{NMR}\left(75 \mathrm{MHz}, \mathrm{CDCl}_{3}\right) \delta=161.9(\mathrm{C}), 144.6(\mathrm{C}), 140.6(\mathrm{C}), 137.3(\mathrm{C}), 129.3(2 \times \mathrm{CH}), 128.8(2$ x CH), $127.7(2 \times \mathrm{CH}), 126.8(\mathrm{CH}), 125.5(2 \times \mathrm{CH}), 116.2(\mathrm{CH}), 92.0(\mathrm{CH}), 59.5(\mathrm{CH}), 26.8(3 \times$ $\left.\mathrm{CH}_{3}\right), 21.3\left(\mathrm{CH}_{3}\right), 16.7(\mathrm{C}),-6.2\left(\mathrm{CH}_{3}\right),-6.3\left(\mathrm{CH}_{3}\right)$.

HRMS (ESI) m/z: [M + H] ${ }^{+}$Calcd. for $\mathrm{C}_{23} \mathrm{H}_{31} \mathrm{OSi}$ : 351.2127; Found: 351.2139. 
Trans-tert-butyl(5-(4-(tert-butyl)phenyl)-4-phenyl-4,5-dihydrofuran-2-yl)dimethylsilane (4c).

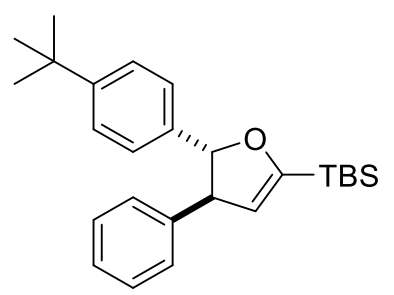

Yield: 98\%, $77 \mathrm{mg}$

Colorless oil

$\mathrm{R}_{\mathrm{f}}\left(\mathrm{SiO}_{2}\right)=0.24($ Hexanes/ Ethyl acetate, $(20: 1))$.

${ }^{1} \mathrm{H}$ NMR (300 MHz, $\left.\mathrm{CDCl}_{3}\right) \delta$ 7.52-7.32 (m, 5H), 7.31-7.19 (m, 4H), 5.37-5.24 (m, 2H), $4.05(\mathrm{dd}, J$ $=7.0$ and $2.5 \mathrm{~Hz}, 1 \mathrm{H}), 1.36(\mathrm{~s}, 9 \mathrm{H}), 1.07(\mathrm{~s}, 9 \mathrm{H}), 0.25(\mathrm{~d}, J=8.9 \mathrm{~Hz}, 6 \mathrm{H})$.

${ }^{13} \mathrm{C}$ NMR $\left(75 \mathrm{MHz}, \mathrm{CDCl}_{3}\right) \delta 161.9(\mathrm{C}), 150.4(\mathrm{C}), 144.7(\mathrm{C}), 140.6(\mathrm{C}), 128.8(2 \mathrm{x} \mathrm{CH}), 127.8(2 \mathrm{x}$ $\mathrm{CH}), 126.8(\mathrm{CH}), 125.5(2 \times \mathrm{CH}), 125.1(2 \times \mathrm{CH}), 116.2(\mathrm{CH}), 91.9(\mathrm{CH}), 59.4(\mathrm{CH}), 34.7(\mathrm{C}), 31.5$ (3 $\left.\mathrm{x} \mathrm{CH}_{3}\right), 26.8\left(3 \times \mathrm{CH}_{3}\right), 16.7(\mathrm{C}),-6.3\left(2 \times \mathrm{CH}_{3}\right)$.

HRMS (ESI) m/z: [M + H] ${ }^{+}$Calcd. for $\mathrm{C}_{26} \mathrm{H}_{37} \mathrm{OSi}$ : 393.2608; Found: 393.2611 .

\section{Trans-(5-([1,1'-biphenyl]-4-yl)-4-phenyl-4,5-dihydrofuran-2-yl)(tert-} butyl)dimethylsilane (4d).

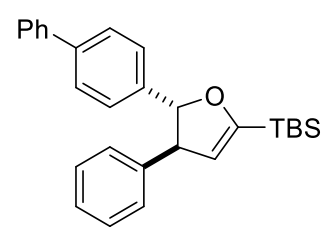

Yield: $97 \%, 80 \mathrm{mg}$

Colorless oil

$\mathrm{R}_{\mathrm{f}}\left(\mathrm{SiO}_{2}\right)=0.16($ Hexanes/ Ethyl acetate, $(200: 1))$.

${ }^{1} \mathrm{H}$ NMR $\left(300 \mathrm{MHz}, \mathrm{CDCl}_{3}\right) \delta$ 7.68-7.57 (m, 4H), $7.48(\mathrm{t}, J=7.4 \mathrm{~Hz}, 2 \mathrm{H}), 7.44-7.36(\mathrm{~m}, 5 \mathrm{H}), 7.34-$ $7.26(\mathrm{~m}, 3 \mathrm{H}), 5.38(\mathrm{~d}, J=7.1 \mathrm{~Hz}, 1 \mathrm{H}), 5.33(\mathrm{~d}, J=2.4 \mathrm{~Hz}, 1 \mathrm{H}), 4.10(\mathrm{dd}, J=7.1,2.4 \mathrm{~Hz}, 1 \mathrm{H}), 1.09$ $(\mathrm{s}, 9 \mathrm{H}), 0.29(3 \mathrm{H}), 0.26(3 \mathrm{H})$.

${ }^{13} \mathrm{C} \mathrm{NMR}\left(75 \mathrm{MHz}, \mathrm{CDCl}_{3}\right) \delta 162.3(\mathrm{C}), 148.8(\mathrm{C}), 142.9(\mathrm{C}), 141.4(\mathrm{C}), 140.8(\mathrm{C}), 129.2(4 \times \mathrm{CH})$, $128.1(2 \times \mathrm{CH}), 127.7(2 \times \mathrm{CH}), 127.7(\mathrm{CH}), 127.5(2 \times \mathrm{CH}), 127.2(\mathrm{CH}), 126.2(\mathrm{CH}), 116.6(\mathrm{CH})$, $92.1(\mathrm{CH}), 59.9(\mathrm{CH}), 27.1\left(3 \mathrm{x} \mathrm{CH}_{3}\right), 17.0(\mathrm{C}),-6.0\left(2 \mathrm{x} \mathrm{CH}_{3}\right)$.

HRMS (ESI) m/z: [M + H] ${ }^{+}$Calcd. for $\mathrm{C}_{28} \mathrm{H}_{33} \mathrm{OSi}$ : 413.2295; Found: 413.2284. 

(4e).

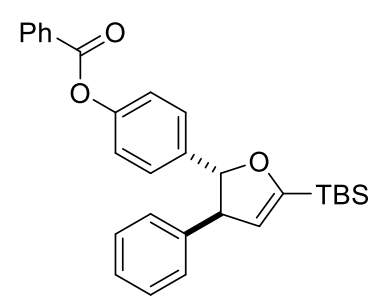

Yield: 99\%, $90 \mathrm{mg}$

Colorless oil

$\mathrm{R}_{\mathrm{f}}\left(\mathrm{SiO}_{2}\right)=0.15($ Hexanes/ Ethyl acetate, $(10: 1))$.

${ }^{1} \mathrm{H}$ NMR $\left(300 \mathrm{MHz}, \mathrm{CDCl}_{3}\right) \delta 8.31-8.18(\mathrm{~m}, 2 \mathrm{H}), 7.73-7.62(\mathrm{~m}, 1 \mathrm{H}), 7.55(\mathrm{t}, J=7.5 \mathrm{~Hz}, 2 \mathrm{H}), 7.43-$ $7.35(\mathrm{~m}, 4 \mathrm{H}), 7.343-7.20(\mathrm{~m}, 5 \mathrm{H}), 5.36(\mathrm{~d}, J=7.1 \mathrm{~Hz}, 1 \mathrm{H}), 5.31(\mathrm{~d}, J=2.5 \mathrm{~Hz}, 1 \mathrm{H}), 4.07(\mathrm{dd}, J=$ 7.1 and $2.4 \mathrm{~Hz}, 1 \mathrm{H}), 1.07$ (s, 9H), 0.27 (s, 3H), 0.24 (s, 3H).

${ }^{13} \mathrm{C}$ NMR $\left(75 \mathrm{MHz}, \mathrm{CDCl}_{3}\right) \delta 165.3(\mathrm{C}), 161.8(\mathrm{C}), 150.2(\mathrm{C}), 144.2(\mathrm{C}), 141.1(\mathrm{C}), 133.6(\mathrm{CH})$, $130.2(2 \times \mathrm{CH}), 129.6(\mathrm{C}), 128.8(2 \times \mathrm{CH}), 128.6(2 \times \mathrm{CH}), 127.6(2 \times \mathrm{CH}), 126.9(\mathrm{CH}), 126.5(2 \times$ $\mathrm{CH}), 121.7(2 \times \mathrm{CH}), 116.2(\mathrm{CH}), 91.4(\mathrm{CH}), 59.4(\mathrm{CH}), 26.6\left(3 \times \mathrm{CH}_{3}\right), 16.6(\mathrm{C}),-6.4\left(\mathrm{CH}_{3}\right),-6.4$ $\left(\mathrm{CH}_{3}\right)$.

HRMS (ESI) m/z: [M + H $]^{+}$Calcd. for $\mathrm{C}_{29} \mathrm{H}_{33} \mathrm{O}_{3} \mathrm{Si}$ : 457.2193; Found: 457.2198.

Trans-tert-butyl(5-(4-chlorophenyl)-4-phenyl-4,5-dihydrofuran-2-yl)dimethylsilane (4f)

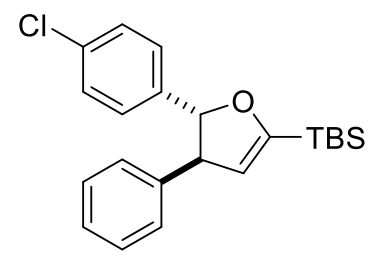

Yield: $99 \%, 73 \mathrm{mg}$

Colorless oil

$\mathrm{R}_{\mathrm{f}}\left(\mathrm{SiO}_{2}\right)=0.20$ (Hexanes/ Ethyl acetate, (200:1)).

${ }^{1} \mathrm{H}$ NMR $\left(300 \mathrm{MHz}, \mathrm{CDCl}_{3}\right) \delta=7.45-7.18(\mathrm{~m}, 9 \mathrm{H})$, 5.37-5.21 (m, 2H), 4.06-3.96 (m, 1H), $1.06(\mathrm{~s}$, $9 \mathrm{H}), 0.26(\mathrm{~s}, 3 \mathrm{H}), 0.24(\mathrm{~s}, 3 \mathrm{H})$.

${ }^{13} \mathrm{C} \mathrm{NMR}\left(75 \mathrm{MHz}, \mathrm{CDCl}_{3}\right) \delta=162.0(\mathrm{C}), 144.1(\mathrm{C}), 142.0(\mathrm{C}), 133.3(\mathrm{C}), 128.9(2 \times \mathrm{CH}), 128.8(2$ x CH), $127.7(2 \times \mathrm{CH}), 127.1(\mathrm{CH}), 126.9(2 \times \mathrm{CH}), 116.3(\mathrm{CH}), 91.4(\mathrm{CH}), 59.6(\mathrm{CH}), 26.7(3 \times$ $\left.\mathrm{CH}_{3}\right), 16.7(\mathrm{C}),-6.2\left(\mathrm{CH}_{3}\right),-6.3\left(\mathrm{CH}_{3}\right)$.

HRMS (ESI) m/z: $[\mathrm{M}+\mathrm{H}]^{+}$Calcd. for $\mathrm{C}_{22} \mathrm{H}_{28} \mathrm{ClOSi}$ : 371.1591; Found: 371.1594 
Trans-tert-butyldimethyl(5-(p-bromophenyl)-4-phenyl-4,5-dihydrofuran-2-yl)silane (4g).

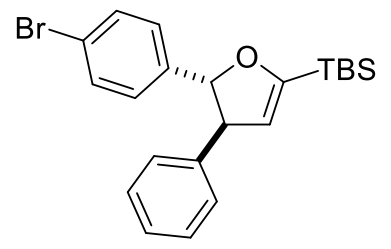

Yield: $99 \%, 82 \mathrm{mg}$.

Colorless oil

$\mathrm{R}_{\mathrm{f}}\left(\mathrm{SiO}_{2}\right)=0.21($ Hexanes/ Ethyl acetate, (200:1)).

${ }^{1} \mathrm{H}$ NMR (300 MHz, $\left.\mathrm{CDCl}_{3}\right) \delta=7.54-7.43(\mathrm{~m}, 2 \mathrm{H}), 7.42-7.15(\mathrm{~m}, 7 \mathrm{H}), 5.38-5.15(\mathrm{~m}, 2 \mathrm{H}), 3.99$ (dd, $J(\mathrm{H}, \mathrm{H})=7.2$ and $2.5 \mathrm{~Hz}, 1 \mathrm{H}), 1.06(\mathrm{~s}, 9 \mathrm{H}), 0.25(\mathrm{~s}, 3 \mathrm{H}), 0.23(\mathrm{~s}, 3 \mathrm{H})$.

${ }^{13} \mathrm{C}$ NMR $\left(75 \mathrm{MHz}, \mathrm{CDCl}_{3}, 25^{\circ} \mathrm{C}\right) \delta=162.0(\mathrm{C}), 144.1(\mathrm{C}), 142.6(\mathrm{C}), 131.7(2 \mathrm{x} \mathrm{CH}), 128.9(2 \mathrm{x}$ $\mathrm{CH}), 127.7(2 \times \mathrm{CH}), 127.2(2 \times \mathrm{CH}), 127.1(\mathrm{CH}), 121.4(\mathrm{C}), 116.3(\mathrm{CH}), 91.4(\mathrm{CH}), 59.6(\mathrm{CH})$, $26.7\left(3 \times \mathrm{CH}_{3}\right), 16.7(\mathrm{C}),-6.3\left(\mathrm{CH}_{3}\right),-6.3\left(\mathrm{CH}_{3}\right)$.

HRMS (ESI) m/z: $[\mathrm{M}+\mathrm{H}]^{+}$Calcd. for $\mathrm{C}_{22} \mathrm{H}_{28}$ BrOSi: 415.1093; Found: 415.1087.

Trans-tert-butyl(5-(4-fluorophenyl)-4-phenyl-4,5-dihydrofuran-2-yl)dimethylsilane (4h).

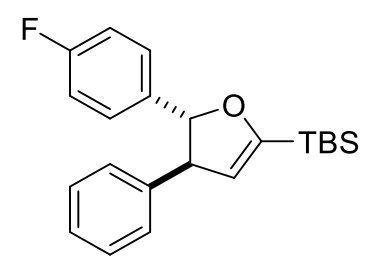

Yield: 97\%, $69 \mathrm{mg}$

Colorless oil

$\mathrm{R}_{\mathrm{f}}\left(\mathrm{SiO}_{2}\right)=0.31$ (Hexanes/ Ethyl acetate, (200:1)).

${ }^{1} \mathrm{H}$ NMR (300 MHz, $\left.\mathrm{CDCl}_{3}\right) \delta$ 7.42- $7.31(\mathrm{~m}, 2 \mathrm{H}), 7.33-7.18(\mathrm{~m}, 5 \mathrm{H}), 7.12-6.99(\mathrm{~m}, 2 \mathrm{H}), 5.35-5.21$ $(\mathrm{m}, 2 \mathrm{H}), 4.00(\mathrm{q}, J=2.5 \mathrm{~Hz}, 1 \mathrm{H}), 1.06(\mathrm{~s}, 9 \mathrm{H}), 0.25(\mathrm{~s}, 3 \mathrm{H}), 0.23(\mathrm{~s}, 3 \mathrm{H})$.

${ }^{13} \mathrm{C} \mathrm{NMR}\left(75 \mathrm{MHz}, \mathrm{CDCl}_{3}\right) \delta 162.2(\mathrm{CF}, \mathrm{d}, J=265.2 \mathrm{~Hz}), 161.8(\mathrm{C}), 144.1(\mathrm{C}), 139.2(\mathrm{C}), 128.8(2$ x CH), $127.6(2 \times \mathrm{CH}), 127.1(\mathrm{CH}), 126.9(2 \times \mathrm{CH}, \mathrm{d}, J=6.9 \mathrm{~Hz}), 116.2(\mathrm{CH}), 115.3(2 \times \mathrm{CH}, \mathrm{d}, J=$ $21.4 \mathrm{~Hz}), 91.4(\mathrm{CH}), 59.5(\mathrm{CH}), 26.6\left(3 \mathrm{x} \mathrm{CH}_{3}\right), 16.5(\mathrm{C}),-6.5\left(\mathrm{CH}_{3}\right),-6.5\left(\mathrm{CH}_{3}\right)$.

${ }^{19} \mathrm{~F}$ NMR $\left(282 \mathrm{MHz}, \mathrm{CDCl}_{3}\right) \delta-115.2$.

HRMS (ESI) m/z: $[\mathrm{M}+\mathrm{H}]^{+}$Calcd. for $\mathrm{C}_{22} \mathrm{H}_{28}$ FOSi: 355.1888; Found: 355.1889 . 
Methyl 4-(trans-5-(tert-butyldimethylsilyl)-3-phenyl-2,3-dihydrofuran-2-yl)benzoate (4i).

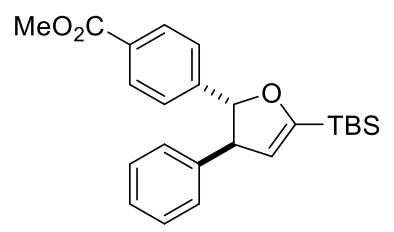

Yield: $98 \%, 77 \mathrm{mg}$

Colorless oil

$\mathrm{R}_{\mathrm{f}}\left(\mathrm{SiO}_{2}\right)=0.23($ Hexanes/ Ethyl acetate, $(10: 1))$.

${ }^{1} \mathrm{H}$ NMR $\left(300 \mathrm{MHz}, \mathrm{CDCl}_{3}\right) \delta=7.93(\mathrm{~d}, J=8.0 \mathrm{~Hz}, 2 \mathrm{H}), 7.33-7.07(\mathrm{~m}, 7 \mathrm{H}), 5.25(\mathrm{~d}, J=7.1 \mathrm{~Hz}$, $1 \mathrm{H}), 5.17(\mathrm{~d}, J=2.5 \mathrm{~Hz}, 1 \mathrm{H}), 3.89(\mathrm{dd}, J=7.0,2.5 \mathrm{~Hz}, 1 \mathrm{H}), 3.82(\mathrm{~s}, 3 \mathrm{H}), 0.95(\mathrm{~s}, 10 \mathrm{H}), 0.15$ (s, 3H), $0.12(\mathrm{~s}, 3 \mathrm{H})$.

${ }^{13} \mathrm{C}$ NMR $\left(75 \mathrm{MHz}, \mathrm{CDCl}_{3}\right) \delta=167.1(\mathrm{C}), 162.0(\mathrm{C}), 148.6(\mathrm{C}), 144.0(\mathrm{C}), 130.0(2 \times \mathrm{CH}), 129.4$ (C), $128.9(2 \times \mathrm{CH}), 127.7(2 \times \mathrm{CH}), 127.1(\mathrm{CH}), 125.3(2 \times \mathrm{CH}), 116.3(\mathrm{CH}), 91.5(\mathrm{CH}), 59.6(\mathrm{CH})$, $52.2\left(\mathrm{CH}_{3}\right), 26.7\left(3 \times \mathrm{CH}_{3}\right), 16.7(\mathrm{C}),-6.3\left(\mathrm{CH}_{3}\right),-6.3\left(\mathrm{CH}_{3}\right)$.

HRMS (ESI) m/z: [M + H] $]^{+}$Calcd. for $\mathrm{C}_{24} \mathrm{H}_{31} \mathrm{O}_{3} \mathrm{Si}$ : 395.2037; Found: 395.2038.

Trans-tert-butyldimethyl(4-phenyl-5-(4-(trifluoromethyl)phenyl)-4,5-dihydrofuran-2yl)silane (4j)

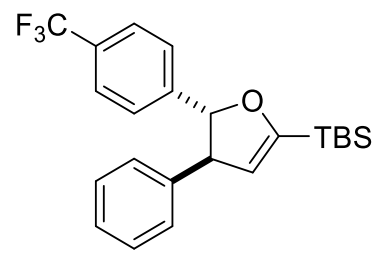

Yield: 99\%, $80 \mathrm{mg}((3: 1)$ mixture diastereoisomers).

Data for pure trans-isomer synthesized from alkynol anti-5j. Colorless oil

$\mathrm{R}_{\mathrm{f}}\left(\mathrm{SiO}_{2}\right)=0.24$ (Hexanes/ Ethyl acetate, (200:1)).

${ }^{1} \mathrm{H}$ NMR $\left(300 \mathrm{MHz}, \mathrm{CDCl}_{3}\right) \delta=7.68-7.59(\mathrm{~m}, 2 \mathrm{H}), 7.47-7.21(\mathrm{~m}, 7 \mathrm{H}), 5.37(\mathrm{~d}, J=7.1 \mathrm{~Hz}, 1 \mathrm{H})$, $5.30(\mathrm{~d}, J=2.5 \mathrm{~Hz}, 1 \mathrm{H}), 4.00(\mathrm{dd}, J=7.2,2.5 \mathrm{~Hz}, 1 \mathrm{H}), 1.07$ (s, 9H), 0.27 (s, 3H), 0.25 (s, 3H).

${ }^{13} \mathrm{C} \mathrm{NMR}\left(75 \mathrm{MHz}, \mathrm{CDCl}_{3}\right) \delta=162.1(\mathrm{C}), 147.5(\mathrm{C}), 143.9(\mathrm{C}), 129.0(2 \times \mathrm{CH}), 127.8(2 \times \mathrm{CH})$, $125.7(2 \times \mathrm{CH}), 125.7(2 \times \mathrm{CH}), 125.7(\mathrm{CH}), 127.2(\mathrm{C}), 116.3(\mathrm{CH}), 91.3(\mathrm{CH}), 59.7(\mathrm{CH}), 26.7(3 \times$ $\left.\mathrm{CH}_{3}\right), 16.7(\mathrm{C}),-6.3\left(\mathrm{CH}_{3}\right),-6.3\left(\mathrm{CH}_{3}\right)$.

${ }^{19} \mathrm{~F} \mathrm{NMR}\left(282 \mathrm{MHz}, \mathrm{CDCl}_{3}\right) \delta=-62.4$.

HRMS (ESI) m/z: [M + Na $]^{+}$Calcd. for $\mathrm{C}_{23} \mathrm{H}_{27} \mathrm{~F}_{3} \mathrm{NaOSi}$ : 427.1675; Found: 427.1664. 
Trans-tert-butyldimethyl(5-(4-nitrophenyl)-4-phenyl-4,5-dihydrofuran-2-yl)silane (4k).

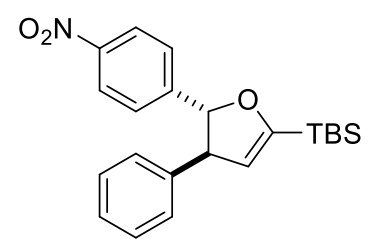

Yield: 77\%, 59 mg. ((1.5:1) mixture diastereoisomers).

Data for pure trans-isomer synthesized from alkynol anti-5p. Yellowish oil

$\operatorname{Rf}\left(\mathrm{SiO}_{2}\right)=0.07($ Hexanes/ Ethyl acetate, $(200: 1))$.

${ }^{1} \mathrm{H}$ NMR $\left(300 \mathrm{MHz}, \mathrm{CDCl}_{3}\right) \delta=8.23-8.15(\mathrm{~m}, 2 \mathrm{H}), 7.46-7.14(\mathrm{~m}, 7 \mathrm{H}), 5.36(\mathrm{~d}, J=7.2 \mathrm{~Hz}, 1 \mathrm{H})$, $5.25(\mathrm{~d}, J=2.5 \mathrm{~Hz}, 1 \mathrm{H}), 3.94(\mathrm{dd}, J=7.3$ and $2.5 \mathrm{~Hz}, 1 \mathrm{H}), 1.02(\mathrm{~s}, 9 \mathrm{H}), 0.23(\mathrm{~s}, 3 \mathrm{H}), 0.20$ (s, 3H).

${ }^{13} \mathrm{C} \mathrm{NMR}\left(75 \mathrm{MHz}, \mathrm{CDCl}_{3}\right) \delta=162.1(\mathrm{C}), 150.7(\mathrm{C}), 147.5(\mathrm{C}), 143.5(\mathrm{C}), 129.1(2 \times \mathrm{CH}), 127.7(2$ x CH), $127.3(\mathrm{CH}), 126.1(2 \times \mathrm{CH}), 124.0(2 \times \mathrm{CH}), 116.4(\mathrm{CH}), 90.9(\mathrm{CH}), 59.6(\mathrm{CH}), 26.7(3 \times$ $\left.\mathrm{CH}_{3}\right), 16.6(\mathrm{C}),-6.3\left(2 \times \mathrm{CH}_{3}\right)$.

HRMS (ESI) m/z: $[\mathrm{M}+\mathrm{H}]^{+}$Calcd. for $\mathrm{C}_{22} \mathrm{H}_{28} \mathrm{NO}_{3} \mathrm{Si}$ : 382.1833; Found: 382.1835 .

Trans-tert-butyldimethyl(4-phenyl-5-(m-tolyl)-4,5-dihydrofuran-2-yl)silane (4l).

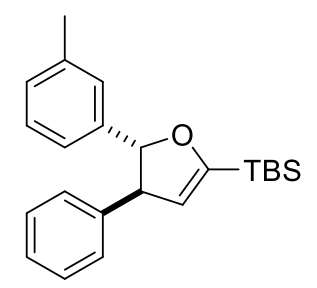

Yield: 99\%, $69 \mathrm{mg}$

Colorless oil

$\mathrm{R}_{\mathrm{f}}\left(\mathrm{SiO}_{2}\right)=0.23$ (Hexanes/ Ethyl acetate, (20:1)).

${ }^{1} \mathrm{H}$ NMR $\left(300 \mathrm{MHz}, \mathrm{CDCl}_{3}\right) \delta$ 7.46-7.33 (m, 2H), 7.33-7.21 (m, 4H), 7.19-7.08 (m, 3H), 5.33-5.25 $(\mathrm{m}, 2 \mathrm{H}), 4.04(\mathrm{dd}, J=6.9$ and $2.4 \mathrm{~Hz}, 1 \mathrm{H}), 2.38(\mathrm{~s}, 3 \mathrm{H}), 1.07(\mathrm{~s}, 10 \mathrm{H}), 0.27(\mathrm{~s}, 3 \mathrm{H}), 0.24$ (s, 3H).

${ }^{13} \mathrm{C}$ NMR $\left(75 \mathrm{MHz}, \mathrm{CDCl}_{3}\right) \delta 161.8(\mathrm{C}), 144.5(\mathrm{C}), 143.5(\mathrm{C}), 138.1(\mathrm{~s}), 128.7$ (2 X CH), 128.4 $(\mathrm{CH}), 128.2(\mathrm{CH}), 127.6(2 \times \mathrm{CH}), 126.7(\mathrm{CH}), 126.0(\mathrm{CH}), 122.4(\mathrm{CH}), 116.2(\mathrm{CH}), 91.9(\mathrm{CH})$, $59.4(\mathrm{CH}), 26.6\left(\mathrm{CH}_{3}\right), 21.5\left(3 \times \mathrm{CH}_{3}\right), 16.6(\mathrm{C}),-6.4\left(2 \times \mathrm{CH}_{3}\right)$.

HRMS (ESI) m/z: [M + H] ${ }^{+}$Calcd. for $\mathrm{C}_{23} \mathrm{H}_{31} \mathrm{OSi}$ : 351.2139; Found: 351.2143. 
Trans-tert-butyl(5-(3-methoxyphenyl)-4-phenyl-4,5-dihydrofuran-2-yl)dimethylsilane (4m).

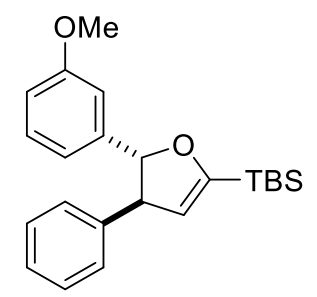

Yield: $98 \%, 72 \mathrm{mg}$

Colorless oil

$\mathrm{R}_{\mathrm{f}}\left(\mathrm{SiO}_{2}\right)=0.18$ (Hexanes/ Ethyl acetate, (200:1)).

${ }^{1} \mathrm{H}$ NMR $\left(300 \mathrm{MHz}, \mathrm{CDCl}_{3}\right) \delta$ 7.54-7.14 (m, 6H), 6.98-6.73 (m, 3H), 5.40-5.21 (m, 2H), $4.03(\mathrm{~d}, J=$ $6.8 \mathrm{~Hz}, 1 \mathrm{H}), 3.82(\mathrm{~s}, 3 \mathrm{H}), 1.07$ (s, 9H), $0.26(\mathrm{~s}, 3 \mathrm{H}) 0.26(\mathrm{~s}, 3 \mathrm{H})$.

${ }^{13} \mathrm{C}$ NMR (75 MHz, $\left.\mathrm{CDCl}_{3}\right) \delta 161.9(\mathrm{C}), 159.9(\mathrm{C}), 145.3(\mathrm{C}), 144.5(\mathrm{C}), 129.7(\mathrm{CH}), 128.8(2 \mathrm{x}$ $\mathrm{CH}), 127.7(2 \times \mathrm{CH}), 126.9(\mathrm{CH}), 117.7(\mathrm{CH}), 116.3(\mathrm{CH}), 113.3(\mathrm{CH}), 110.5(\mathrm{CH}), 91.9(\mathrm{CH}), 59.6$ $(\mathrm{CH}), 55.3\left(\mathrm{CH}_{3}\right), 26.8\left(3 \times \mathrm{CH}_{3}\right), 16.7(\mathrm{C}),-6.3\left(2 \times \mathrm{CH}_{3}\right)$.

HRMS (ESI) m/z: $[\mathrm{M}+\mathrm{H}]^{+}$Calcd. for $\mathrm{C}_{23} \mathrm{H}_{31} \mathrm{O}_{2} \mathrm{Si}$ : 367.2091; Found: 367.2088.

Trans-tert-butyl(5-(3-chlorophenyl)-4-phenyl-4,5-dihydrofuran-2-yl)dimethylsilane (4n).<smiles>CC(C)(C)C1=CC(c2ccccc2)OC1c1cccc(Cl)c1</smiles>

Yield: 99\%, $73 \mathrm{mg}$

Colorless oil

$\mathrm{R}_{\mathrm{f}}\left(\mathrm{SiO}_{2}\right)=0.27$ (Hexanes/ Ethyl acetate, (200:1)).

${ }^{1} \mathrm{H}$ NMR $\left(300 \mathrm{MHz}, \mathrm{CDCl}_{3}\right) \delta=7.45-7.21(\mathrm{~m}, 8 \mathrm{H}), 7.19-7.14(\mathrm{~m}, 1 \mathrm{H}), 5.30(\mathrm{~d}, J=1.4 \mathrm{~Hz}, 1 \mathrm{H})$, $5.28(\mathrm{~d}, J=2.9 \mathrm{~Hz}, 1 \mathrm{H}), 4.00(\mathrm{dd}, J=6.9,2.5 \mathrm{~Hz}, 1 \mathrm{H}), 1.07$ (s, 9H), 0.27 (s, 3H), 0.24 (s, 3H).

${ }^{13} \mathrm{C}$ NMR $\left(75 \mathrm{MHz}, \mathrm{CDCl}_{3}\right) \delta=162.0(\mathrm{C}), 145.6(\mathrm{C}), 144.1(\mathrm{C}), 134.5(\mathrm{C}), 130.0(\mathrm{CH}), 128.9(2 \mathrm{x}$ $\mathrm{CH}), 127.7(\mathrm{CH}), 127.6(2 \times \mathrm{CH}), 127.1(\mathrm{CH}), 125.6(\mathrm{CH}), 123.6(\mathrm{CH}), 116.3(\mathrm{CH}), 91.2(\mathrm{CH}), 59.5$ $(\mathrm{CH}), 26.7\left(3 \times \mathrm{CH}_{3}\right), 16.7(\mathrm{C}),-6.3\left(2 \times \mathrm{CH}_{3}\right)$.

HRMS (ESI) m/z: $[\mathrm{M}+\mathrm{H}]^{+}$Calcd. for $\mathrm{C}_{22} \mathrm{H}_{28} \mathrm{ClOSi}$ : 371.1592; Found: 371.1584 . 
Trans-tert-butyl(5-(3,5-dimethylphenyl)-4-phenyl-4,5-dihydrofuran-2-yl)dimethylsilane (4o).

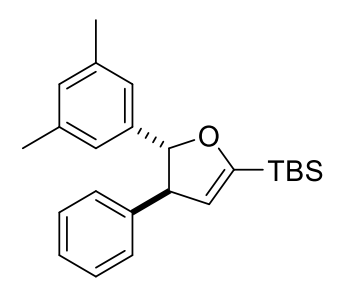

Yield: $97 \%, 71 \mathrm{mg}$

Colorless oil

$\mathrm{R}_{\mathrm{f}}\left(\mathrm{SiO}_{2}\right)=0.31($ Hexanes/ Ethyl acetate, $(200: 1))$.

${ }^{1} \mathrm{H}$ NMR (300 MHz, $\left.\mathrm{CDCl}_{3}\right) \delta=7.44-7.33(\mathrm{~m}, 2 \mathrm{H}), 7.33-7.23(\mathrm{~m}, 3 \mathrm{H}), 6.96(\mathrm{~d}, J=1.1 \mathrm{~Hz}, 3 \mathrm{H}), 5.31$ $(\mathrm{d}, J=2.5 \mathrm{~Hz}, 1 \mathrm{H}), 5.28(\mathrm{~d}, J=6.7 \mathrm{~Hz}, 1 \mathrm{H}), 4.04(\mathrm{dd}, J=6.7,2.5 \mathrm{~Hz}, 1 \mathrm{H}), 2.35(\mathrm{~s}, 6 \mathrm{H}), 1.09$ (s, $9 \mathrm{H}), 0.28(\mathrm{~s}, 3 \mathrm{H}), 0.25(\mathrm{~s}, 3 \mathrm{H})$.

${ }^{13} \mathrm{C} \mathrm{NMR}\left(75 \mathrm{MHz}, \mathrm{CDCl}_{3}\right) \delta=161.9(\mathrm{C}), 144.8(\mathrm{C}), 143.6(\mathrm{C}), 138.1(2 \times \mathrm{C}), 129.2(\mathrm{CH}), 128.8(2$ x CH), $127.7(2 \times \mathrm{CH}), 126.8(\mathrm{CH}), 123.3(2 \times \mathrm{CH}), 116.3(\mathrm{CH}), 92.1(\mathrm{CH}), 59.4(\mathrm{CH}), 26.8(3 \mathrm{x}$ $\left.\mathrm{CH}_{3}\right), 21.5\left(2 \times \mathrm{CH}_{3}\right), 16.7(\mathrm{C}),-6.3\left(2 \times \mathrm{CH}_{3}\right)$.

HRMS (ESI) m/z: [M + H] ${ }^{+}$Calcd. for $\mathrm{C}_{24} \mathrm{H}_{33} \mathrm{OSi}$ : 365.2295; found: 365.2297.

Trans-tert-butyl(5-(3,5-dibromophenyl)-4-phenyl-4,5-dihydrofuran-2-yl)dimethylsilane (4p).

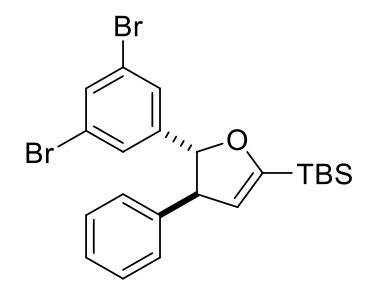

Yield: 99\%, $97 \mathrm{mg}((2: 1)$ mixture diastereoisomers).

Data for pure trans-isomer synthesized from alkynol anti-5p.

Colorless oil

$\mathrm{R}_{\mathrm{f}}\left(\mathrm{SiO}_{2}\right)=0.26($ Hexanes/ Ethyl acetate, $(200: 1))$.

${ }^{1} \mathrm{H}$ NMR $\left(300 \mathrm{MHz}, \mathrm{CDCl}_{3}\right) \delta=7.60(\mathrm{t}, J=1.8 \mathrm{~Hz}, 1 \mathrm{H}), 7.44-7.19(\mathrm{~m}, 7 \mathrm{H}), 5.29(\mathrm{~d}, J=2.5 \mathrm{~Hz}$, $1 \mathrm{H}), 5.24(\mathrm{~d}, J=6.3 \mathrm{~Hz}, 1 \mathrm{H}), 3.96(\mathrm{dd}, J=6.4,2.6 \mathrm{~Hz}, 1 \mathrm{H}), 1.06(\mathrm{~s}, 9 \mathrm{H}), 0.27(\mathrm{~s}, 3 \mathrm{H}), 0.24(\mathrm{~s}, 3 \mathrm{H})$.

${ }^{13} \mathrm{C} \mathrm{NMR}\left(75 \mathrm{MHz}, \mathrm{CDCl}_{3}\right) \delta=161.9(\mathrm{C}), 147.5(\mathrm{C}), 143.6(\mathrm{C}), 133.1(\mathrm{CH}), 129.0(2 \times \mathrm{CH}), 127.6$ $(2 \times \mathrm{CH}), 127.3(3 \times \mathrm{CH}), 123.2(2 \times \mathrm{C}), 116.4(\mathrm{CH}), 90.2(\mathrm{CH}), 59.4(\mathrm{CH}), 26.7\left(3 \times \mathrm{CH}_{3}\right), 16.6(\mathrm{C})$, $-6.3\left(\mathrm{CH}_{3}\right),-6.3\left(\mathrm{CH}_{3}\right)$.

HRMS (ESI) m/z: $[\mathrm{M}+\mathrm{H}]^{+}$Calcd. for $\mathrm{C}_{22} \mathrm{H}_{27} \mathrm{Br}_{2} \mathrm{OSi}$ : 493.0192 ; Found: 493.0173. 
Trans-tert-butyldimethyl(4-phenyl-5-(o-tolyl)-4,5-dihydrofuran-2-yl)silane (4q).

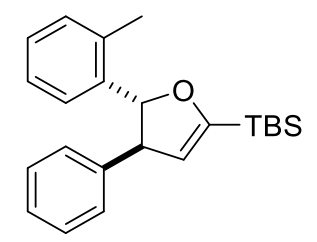

Yield: 97\%, $68 \mathrm{mg}$

Colorless oil

$\mathrm{R}_{\mathrm{f}}\left(\mathrm{SiO}_{2}\right)=0.24$ (Hexanes/ Ethyl acetate, $\left.(200: 1)\right)$.

${ }^{1} \mathrm{H}$ NMR $\left(300 \mathrm{MHz}, \mathrm{CDCl}_{3}\right) \delta$ 7.44-7.33 (m, 3H), 7.33-7.13 (m, 6H), $5.61(\mathrm{~d}, J=6.9 \mathrm{~Hz}, 1 \mathrm{H}), 5.30$ $(\mathrm{d}, J=2.5 \mathrm{~Hz}, 1 \mathrm{H}), 3.97(\mathrm{dd}, J=6.9$ and $2.5 \mathrm{~Hz}, 1 \mathrm{H}), 2.11(\mathrm{~s}, 3 \mathrm{H}), 1.10(\mathrm{~s}, 9 \mathrm{H}), 0.31(\mathrm{~s}, 3 \mathrm{H}), 0.25$ (s, $3 \mathrm{H})$.

${ }^{13} \mathrm{C}$ NMR $\left(75 \mathrm{MHz}, \mathrm{CDCl}_{3}\right) \delta 161.4(\mathrm{C}), 144.7(\mathrm{C}), 113.3(\mathrm{C}), 134.7(\mathrm{C}), 130.6(\mathrm{CH}), 128.8,(2 \mathrm{x}$ CH) $127.7(2 \times \mathrm{CH}), 127.3(\mathrm{CH}), 126.9(\mathrm{CH}), 126.2(\mathrm{CH}), 125.3(\mathrm{CH}), 116.8(\mathrm{CH}), 89.7(\mathrm{CH}), 59.0$ $(\mathrm{CH}), 26.8\left(3 \times \mathrm{CH}_{3}\right), 19.7\left(\mathrm{CH}_{3}\right), 16.7(\mathrm{C}),-6.3\left(2 \times \mathrm{CH}_{3}\right)$.

HRMS (ESI) m/z: $[\mathrm{M}+\mathrm{H}]^{+}$Calcd. for $\mathrm{C}_{23} \mathrm{H}_{31} \mathrm{OSi}$ : 351.2134; Found: 351.2139 .

Trans-tert-butyldimethyl(5-(naphthalen-1-yl)-4-phenyl-4,5-dihydrofuran-2-yl)silane (4r).

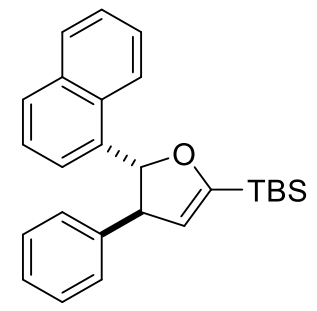

Yield: $98 \%, 76 \mathrm{mg}$

Colorless oil

$\mathrm{R}_{\mathrm{f}}\left(\mathrm{SiO}_{2}\right)=0.19$ (Hexanes/ Ethyl acetate, (200:1)).

${ }^{1} \mathrm{H}$ NMR $\left(300 \mathrm{MHz}, \mathrm{CDCl}_{3}\right) \delta 7.91(\mathrm{~d}, J=8.2 \mathrm{~Hz}, 1 \mathrm{H}), 7.82(\mathrm{dd}, J=7.3$ and $1.9 \mathrm{~Hz}, 1 \mathrm{H}), 7.73(\mathrm{~d}, J$ $=8.5 \mathrm{~Hz}, 1 \mathrm{H}), 7.55-7.22(\mathrm{~m}, 9 \mathrm{H}), 6.03(\mathrm{~d}, J=6.5 \mathrm{~Hz}, 1 \mathrm{H}), 5.36(\mathrm{~d}, J=2.5 \mathrm{~Hz}, 1 \mathrm{H}), 4.11(\mathrm{dd}, J=6.5$ and $2.5 \mathrm{~Hz}, 1 \mathrm{H}), 1.11(\mathrm{~s}, 9 \mathrm{H}), 0.35(\mathrm{~s}, 3 \mathrm{H}), 0.27(\mathrm{~s}, 3 \mathrm{H})$.

${ }^{13} \mathrm{C} \mathrm{NMR}\left(75 \mathrm{MHz}, \mathrm{CDCl}_{3}\right) \delta 161.3(\mathrm{C}), 144.5(\mathrm{C}), 138.3(\mathrm{C}), 134.1(\mathrm{C}), 130.2(\mathrm{C}), 128.8(\mathrm{CH})$, $128.7(2 \times \mathrm{CH}), 128.0(\mathrm{CH}), 127.9(2 \times \mathrm{CH}), 126.9(\mathrm{CH}), 125.7(\mathrm{CH}), 125.5(\mathrm{CH}), 125.4(\mathrm{CH})$, $124.3(\mathrm{CH}), 122.9(\mathrm{CH}), 117.1(\mathrm{CH}), 90.6(\mathrm{CH}), 58.8(\mathrm{CH}), 26.7\left(3 \mathrm{x} \mathrm{CH}_{3}\right), 16.6(\mathrm{C}),-6.3\left(\mathrm{CH}_{3}\right)$, $6.3\left(\mathrm{CH}_{3}\right)$.

HRMS (ESI) m/z: [M + H] ${ }^{+}$Calcd. for $\mathrm{C}_{26} \mathrm{H}_{31} \mathrm{OSi}$ : 387.2139; Found: 387.2137. 
Trans-tert-butyl(-4,5-di-p-tolyl-4,5-dihydrofuran-2-yl)dimethylsilane (4s).

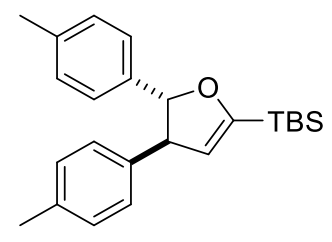

Yield: $99 \%, 72 \mathrm{mg}$

Colorless oil

$\mathrm{R}_{\mathrm{f}}\left(\mathrm{SiO}_{2}\right)=0.24$ (Hexanes/ Ethyl acetate, (200:1)).

${ }^{1} \mathrm{H}$ NMR $\left(300 \mathrm{MHz}, \mathrm{CDCl}_{3}\right) \delta=7.26-7.12(\mathrm{~m}, 8 \mathrm{H}), 5.28(\mathrm{~d}, J=2.5 \mathrm{~Hz}, 1 \mathrm{H}), 5.28(\mathrm{~d}, J=7.1 \mathrm{~Hz}$, $1 \mathrm{H}$ ), 4.01 (dd, $J=7.2$ and $2.5 \mathrm{~Hz}, 1 \mathrm{H}), 2.39$ (s, 6H), 1.07 (s, 9H), 0.26 (s, 3H), 0.24 (s, 3H).

${ }^{13} \mathrm{C}$ NMR (75 MHz, $\left.\mathrm{CDCl}_{3}\right) \delta=161.7$ (C), 141.7 (C), 140.8 (C), 137.2 (C), $136.4(\mathrm{C}), 129.5(2 \mathrm{x}$ $\mathrm{CH}), 129.3(2 \times \mathrm{CH}), 127.6(2 \times \mathrm{CH}), 125.5(2 \times \mathrm{CH}), 116.5(\mathrm{CH}), 92.1(\mathrm{CH}), 59.1(\mathrm{CH}), 26.8(3 \times$ $\left.\mathrm{CH}_{3}\right), 21.3\left(\mathrm{CH}_{3}\right), 21.2\left(\mathrm{CH}_{3}\right), 16.7(\mathrm{C}),-6.2\left(\mathrm{CH}_{3}\right),-6.3\left(\mathrm{CH}_{3}\right)$.

HRMS (ESI) m/z: $[\mathrm{M}+\mathrm{H}]^{+}$Calcd. for $\mathrm{C}_{24} \mathrm{H}_{33} \mathrm{OSi}$ : Not obtained. Unstable compound.

Trans-triisopropyl(4-phenyl-5-(p-tolyl)-4,5-dihydrofuran-2-yl)silane (4t).

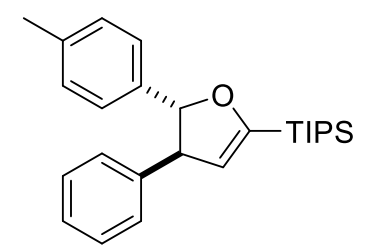

Yield: $99 \%, 78 \mathrm{mg}$

Colorless oil

$\mathrm{R}_{\mathrm{f}}\left(\mathrm{SiO}_{2}\right)=0.19$ (Hexanes/ Ethyl acetate, (200:1)).

${ }^{1} \mathrm{H}$ NMR $\left(300 \mathrm{MHz}, \mathrm{CDCl}_{3}\right) \delta$ 7.40-7.12 (m, 9H), $5.31(\mathrm{~d}, J=2.4 \mathrm{~Hz}, 1 \mathrm{H}), 5.22(\mathrm{~d}, J=8.1 \mathrm{~Hz}, 1 \mathrm{H})$, $4.08(\mathrm{dd}, J=7.9,2.4 \mathrm{~Hz}, 1 \mathrm{H}), 2.37$ (s, 3H), $1.35-1.13(\mathrm{~m}, 21 \mathrm{H})$.

${ }^{13} \mathrm{C}$ NMR $\left(75 \mathrm{MHz}, \mathrm{CDCl}_{3}\right) \delta 160.0(\mathrm{C}), 145.0(\mathrm{C}), 140.8(\mathrm{C}), 137.5(\mathrm{C}), 129.5(2 \mathrm{x} \mathrm{CH}), 129.1(2 \mathrm{x}$ $\mathrm{CH}), 128.1(2 \times \mathrm{CH}), 127.1(\mathrm{CH}), 126.0(2 \times \mathrm{CH}), 117.2(\mathrm{CH}), 92.2(\mathrm{CH}), 59.9(\mathrm{CH}), 21.6\left(\mathrm{CH}_{3}\right)$, $19.1\left(3 \times \mathrm{CH}_{3}\right), 19.1\left(3 \times \mathrm{CH}_{3}\right), 11.3(3 \times \mathrm{CH})$.

HRMS (ESI) m/z: [M + H] $]^{+}$Calcd. for $\mathrm{C}_{26} \mathrm{H}_{37} \mathrm{OSi}$.: 393.3608; Found: 393.2609 . 


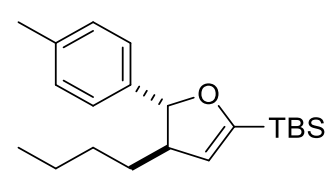

Yield: $98 \%, 65 \mathrm{mg}$

Colorless oil

$\mathrm{R}_{\mathrm{f}}\left(\mathrm{SiO}_{2}\right)=0.30$ (Hexanes/ Ethyl acetate, $\left.(200: 1)\right)$.

${ }^{1} \mathrm{H}$ NMR $\left(300 \mathrm{MHz}, \mathrm{CDCl}_{3}\right) \delta$ 7.37-7.05 (m, 4H), $5.21(\mathrm{~d}, J=2.4 \mathrm{~Hz}, 1 \mathrm{H}), 5.01(\mathrm{~d}, J=7.0 \mathrm{~Hz}, 1 \mathrm{H})$, $2.83(\mathrm{qt}, J=12.3$ and $6.1 \mathrm{~Hz}, 1 \mathrm{H}), 2.37(\mathrm{~s}, 3 \mathrm{H}), 1.69-1.13(\mathrm{~m}, 6 \mathrm{H}), 1.00(\mathrm{~s}, 9 \mathrm{H}), 0.93(\mathrm{t}, 6.5 \mathrm{~Hz})$, $0.19(\mathrm{~s}, 3 \mathrm{H}), 0.17(\mathrm{~s}, 3 \mathrm{H})$.

${ }^{13} \mathrm{C}$ NMR $\left(75 \mathrm{MHz}, \mathrm{CDCl}_{3}\right) \delta 160.4(\mathrm{C}), 141.1(\mathrm{C}), 136.9(\mathrm{C}), 129.0(2 \times \mathrm{CH}), 125.6(2 \times \mathrm{CH}), 116.4$ $(\mathrm{CH}), 89.1(\mathrm{CH}), 53.3(\mathrm{CH}), 35.7\left(\mathrm{CH}_{2}\right), 29.5\left(\mathrm{CH}_{2}\right), 26.6\left(3 \times \mathrm{CH}_{3}\right), 22.8\left(\mathrm{CH}_{2}\right), 21.1\left(\mathrm{CH}_{3}\right), 16.4$ (C), $14.1\left(\mathrm{CH}_{3}\right),-6.5\left(2 \times \mathrm{CH}_{3}\right)$.

HRMS (ESI) m/z: [M + H] $]^{+}$Calcd. for $\mathrm{C}_{21} \mathrm{H}_{35} \mathrm{OSi}$ : Not obtained. Unstable compound.

Trans-tert-butyl(4-cyclopentyl-5-(p-tolyl)-4,5-dihydrofuran-2-yl)dimethylsilane (4v).

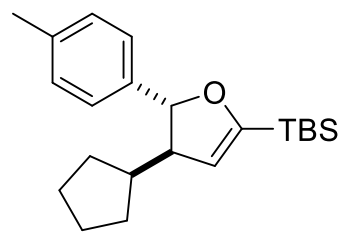

Yield: 97\%, $66 \mathrm{mg}$

Colorless oil

$\mathrm{R}_{\mathrm{f}}\left(\mathrm{SiO}_{2}\right)=0.29($ Hexanes/ Ethyl acetate, $(200: 1))$.

${ }^{1} \mathrm{H}$ NMR $\left(300 \mathrm{MHz}, \mathrm{CDCl}_{3}\right) \delta 7.22(\mathrm{~d}, J=8.0 \mathrm{~Hz}, 2 \mathrm{H}), 7.15(\mathrm{~d}, J=8.0 \mathrm{~Hz}, 2 \mathrm{H}), 5.15(\mathrm{~d}, J=2.5 \mathrm{~Hz}$, $1 \mathrm{H}), 5.10(\mathrm{~d}, J=6.2 \mathrm{~Hz}, 1 \mathrm{H}), 2.79(\mathrm{ddd}, J=7.6,6.3$ and $2.5 \mathrm{~Hz}, 1 \mathrm{H}), 2.47-2.26(\mathrm{~m}, 1 \mathrm{H}), 2.34(\mathrm{~s}$, 3H), 1.99- 1.49 (m, 8H), 0.97 (s, 9H), 0.17 (s, 3H), 0.14(s, 3H).

${ }^{13} \mathrm{C}$ NMR $\left(75 \mathrm{MHz}, \mathrm{CDCl}_{3}\right) \delta 160.7(\mathrm{C}), 141.7(\mathrm{C}), 136.9(\mathrm{C}), 129.2(2 \times \mathrm{CH}), 125.7(2 \times \mathrm{CH}), 115.4$ $(\mathrm{CH}), 88.0(\mathrm{CH}), 58.5(\mathrm{CH}), 45.5(\mathrm{CH}), 30.6\left(\mathrm{CH}_{2}\right), 30.0\left(\mathrm{CH}_{2}\right), 26.7\left(3 \times \mathrm{CH}_{3}\right), 25.5\left(2 \times \mathrm{CH}_{2}\right), 21.3$ $\left(\mathrm{CH}_{3}\right), 16.6(\mathrm{C}),-6.3\left(\mathrm{CH}_{3}\right),-6.4\left(\mathrm{CH}_{3}\right)$.

HRMS (ESI) m/z: [M + H] $]^{+}$Calcd. for $\mathrm{C}_{22} \mathrm{H}_{34} \mathrm{OSi}$ : Not obtained. Unstable compound. 
(E)-tert-butyldimethyl(3-deutero-4-phenyl-5-(p-tolyl)-4,5-dihydrofuran-2-yl)silane (3D4b)

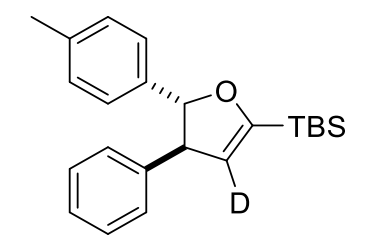

Yield: $97 \%, 68 \mathrm{mg}$

Colorless oil

$\mathrm{R}_{\mathrm{f}}\left(\mathrm{SiO}_{2}\right)=0.24$ (Hexanes/ Ethyl acetate, $\left.(200: 1)\right)$.

${ }^{1} \mathrm{H}$ NMR $\left(400 \mathrm{MHz}, \mathrm{C}_{6} \mathrm{D}_{6}\right) \delta$ 7.25-7.09 (m, 6H), 7.08-7.01 (m, 1H), 7.00-6.90 (m, 2H), $5.37(\mathrm{~d}, J=$ $7.7 \mathrm{~Hz}, 1 \mathrm{H}), 4.04(\mathrm{~d}, J=7.7 \mathrm{~Hz}, 1 \mathrm{H}) 2.07(\mathrm{~s}, 3 \mathrm{H}), 1.07(\mathrm{~s}, 9 \mathrm{H}), 0.20(\mathrm{~s}, 3 \mathrm{H}), 0.18(\mathrm{~s}, 3 \mathrm{H})$.

${ }^{13} \mathrm{C}$ NMR (100 MHz, $\left.\mathrm{C}_{6} \mathrm{D}_{6}\right) \delta 162.0(\mathrm{C}), 144.8(\mathrm{C}), 141.0(\mathrm{C}), 137.2(\mathrm{C}), 129.5(2 \times \mathrm{CH}), 129.0(2 \mathrm{x}$ $\mathrm{CH}), 128.0(2 \times \mathrm{CH}), 127.1(\mathrm{CH}), 126.0(2 \times \mathrm{CH}), 116.5(\mathrm{t}, J=26.1 \mathrm{~Hz}, \mathrm{CD}), 92.8(\mathrm{CH}), 59.9(\mathrm{CH})$, $26.9\left(3 \times \mathrm{CH}_{3}\right), 21.1\left(\mathrm{CH}_{3}\right), 16.8(\mathrm{C}),-6.3\left(2 \times \mathrm{CH}_{3}\right)$.

HRMS (ESI) m/z: $[\mathrm{M}+\mathrm{H}]^{+}$Calcd. for $\mathrm{C}_{23} \mathrm{H}_{30}$ DOSi: 352.2201; Found: 352.2198 .

\section{(E)-tert-butyldimethyl(4-deutero-4-phenyl-5-(p-tolyl)-4,5-dihydrofuran-2-yl)silane (4D-}

4b)

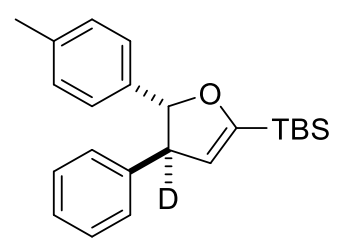

Yield: 94\%, $66 \mathrm{mg}$

Colorless oil

$\mathrm{R}_{\mathrm{f}}\left(\mathrm{SiO}_{2}\right)=0.24$ (Hexanes/ Ethyl acetate, (200:1)).

${ }^{1} \mathrm{H}$ NMR (300 MHz, $\mathrm{CDCl}_{3}$ ) $\delta$ 7.41-7.32 (m, 2H), 7.32-7.15 (m, 7H), 5.29 (s, 2H), 2.38 (s, 3H), 1.06 (s, 9H), 0.26 (s, 3H), 0.23 (s, 3H).

${ }^{13} \mathrm{C} \mathrm{NMR}\left(75 \mathrm{MHz}, \mathrm{CDCl}_{3}\right) \delta 161.8(\mathrm{C}), 144.4(\mathrm{C}), 140.5(\mathrm{C}), 137.1(\mathrm{C}), 129.1(2 \mathrm{x} \mathrm{CH}), 128.7(2 \mathrm{x}$ $\mathrm{CH}), 127.6(2 \times \mathrm{CH}), 126.7(\mathrm{CH}), 125.3(2 \times \mathrm{CH}), 116.0(\mathrm{CH}), 91.8(\mathrm{CH}), 59.2(\mathrm{t}, J=19.5 \mathrm{~Hz}, \mathrm{CD})$, $26.6\left(3 \times \mathrm{CH}_{3}\right), 21.2\left(\mathrm{CH}_{3}\right), 16.6(\mathrm{C}),-6.4\left(2 \times \mathrm{CH}_{3}\right)$.

HRMS (ESI) m/z: $[\mathrm{M}+\mathrm{H}]^{+}$Calcd. for $\mathrm{C}_{23} \mathrm{H}_{30} \mathrm{DOSi}$ : 352.2201; Found: 352.2199 . 


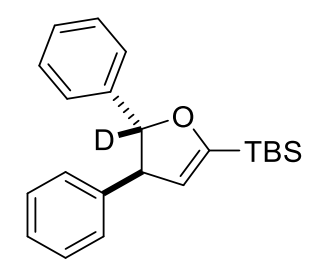

Yield: 98\%, $66 \mathrm{mg}$

Colorless oil

$\mathrm{R}_{\mathrm{f}}\left(\mathrm{SiO}_{2}\right)=0.22($ Hexanes/ Ethyl acetate, $(200: 1))$.

${ }^{1} \mathrm{H}$ NMR (300 MHz, $\left.\mathrm{CDCl}_{3}\right) \delta$ 7.47-7.19 (m, 10H), $5.31(\mathrm{~d}, J=2.4 \mathrm{~Hz}, 1 \mathrm{H}), 4.05(\mathrm{~d}, J=2.3 \mathrm{~Hz}, 1 \mathrm{H})$, $1.08(\mathrm{~s}, 9 \mathrm{H}), 0.28(\mathrm{~s}, 3 \mathrm{H}), 0.25(\mathrm{~s}, 3 \mathrm{H})$.

${ }^{13} \mathrm{C} \mathrm{NMR}\left(75 \mathrm{MHz}, \mathrm{CDCl}_{3}\right) \delta 162.2(\mathrm{C}), 144.8(\mathrm{C}), 143.8(\mathrm{C}), 129.1(2 \times \mathrm{CH}), 128.9(2 \times \mathrm{CH}), 128.0$ $(2 \times \mathrm{CH}), 127.9(\mathrm{CH}), 127.2(\mathrm{CH}), 125.7(2 \times \mathrm{CH}), 116.5(\mathrm{CH}), 91.9(\mathrm{t}, J=21.3 \mathrm{~Hz}, \mathrm{CD}), 59.8(\mathrm{CH})$, $27.0\left(3 \times \mathrm{CH}_{3}\right), 17.0(\mathrm{C}),-6.0\left(2 \times \mathrm{CH}_{3}\right)$.

HRMS (ESI) m/z: $[\mathrm{M}+\mathrm{H}]^{+}$Calcd. for $\mathrm{C}_{22} \mathrm{H}_{28}$ DOSi: 338.22045; Found: 338.2055.

\section{1,3-dimethoxy-6-phenylnaphthalene (6)}

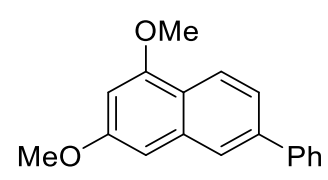

Yield: $88 \%, 46 \mathrm{mg}$

Colorless oil

$\mathrm{R}_{\mathrm{f}}\left(\mathrm{SiO}_{2}\right)=0.31($ Hexanes/ Ethyl acetate, $(20: 1))$.

${ }^{1} \mathrm{H}$ NMR $\left(300 \mathrm{MHz}, \mathrm{CDCl}_{3}\right) \delta 8.23(\mathrm{~d}, J=8.6 \mathrm{~Hz}, 1 \mathrm{H}), 7.92(\mathrm{~d}, J=1.8 \mathrm{~Hz}, 1 \mathrm{H}), 7.74(\mathrm{dt}, J=8.3$, $1.8 \mathrm{~Hz}, 2 \mathrm{H}), 7.61(\mathrm{dd}, J=8.6,1.8 \mathrm{~Hz}, 1 \mathrm{H}), 7.55-7.46(\mathrm{~m}, 2 \mathrm{H}), 7.44-7.36(\mathrm{~m}, 1 \mathrm{H}), 6.82(\mathrm{~d}, J=2.1$ $\mathrm{Hz}, 1 \mathrm{H}), 6.53$ (d, J=2.2 Hz, 1H), 4.02 (s, 3H), 3.96 (s, 3H).

${ }^{13} \mathrm{C} \mathrm{NMR}\left(75 \mathrm{MHz}, \mathrm{CDCl}_{3}\right) \delta=158.6(\mathrm{C}), 156.6(\mathrm{C}), 141.3(\mathrm{C}), 139.7(\mathrm{C}), 135.4(\mathrm{C}), 128.8(2 \mathrm{x}$ $\mathrm{CH}), 128.4(2 \times \mathrm{CH}), 127.3(\mathrm{CH}), 124.5(\mathrm{CH}), 122.6(\mathrm{CH}), 122.6(\mathrm{CH}), 120.9(\mathrm{C}), 98.2(\mathrm{CH}), 97.7$ $(\mathrm{CH}), 55.6\left(\mathrm{CH}_{3}\right), 55.3\left(\mathrm{CH}_{3}\right)$.

HRMS (ESI) m/z: $[\mathrm{M}+\mathrm{H}]^{+}$Calcd. for $\mathrm{C}_{18} \mathrm{H}_{17} \mathrm{O}_{2}$ : 265.1223; Found: 265.1218. 


\section{Experimental procedure for the synthesis of homopropargyl alcohols syn-5 for Hammett plot and anti-5 for the synthesis of pure trans- dihydrofurans $4 \mathrm{j}, 4 \mathrm{k}$ and $4 \mathrm{p}$.}
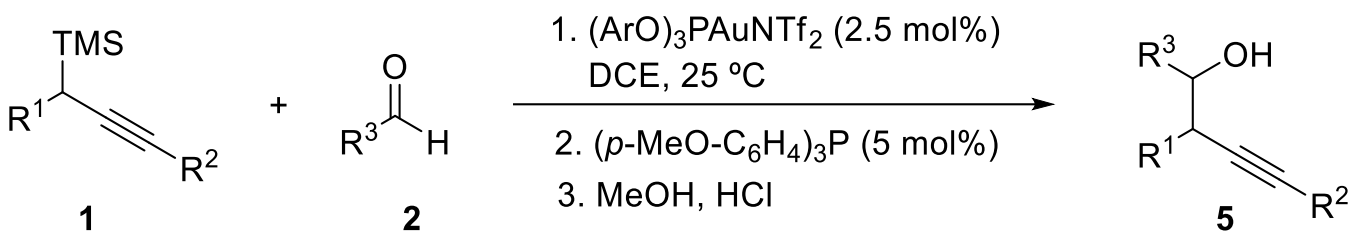

To a mixture of $0.48 \mathrm{mmol}$ of propargylsilane $\mathbf{1}$ and $0.4 \mathrm{mmol}$ of carbonyl compound 2 in dry dichloromethane $(2 \mathrm{~mL})$ at $25^{\circ} \mathrm{C}$, were added $11.2 \mathrm{mg}$ of the gold catalyst $(2.5 \mathrm{~mol} \%)$ and the reaction was then stirred for $50 \mathrm{~min}$. After that time, they were sequentially added $4.1 \mathrm{mg}(0.01$ mmol, $5 \mathrm{~mol} \%$ ) of ( $\left.p-\mathrm{MeO}-\mathrm{C}_{6} \mathrm{H}_{4}\right)_{3} \mathrm{P}$ to deactivate the catalyst, $360 \mu \mathrm{L}$ of dry methanol $(250 \mathrm{mg} ; 8$ mmol; 20 equivalents) and concentrated $\mathrm{HCl}(25 \mu \mathrm{L})$ to deprotect the silylether. The mixture was stirred for $3 \mathrm{~h}$ and then solvents were removed under vacuum. The residue was purified under flash chromatography to separately obtain the corresponding syn or anti homopropargyl alcohols 5 . Homopropargyl alcohols syn-5g was also synthesized at $1 \mathrm{mmol}$ scale and $191 \mathrm{mg}$ (46\%) of compound syn-5g were obtained.

\section{Anti-4-(tert-butyldimethylsilyl)-1-(4-trifluoromethylphenyl)-2-phenylbut-3-yn-1-ol (anti-} 5j)

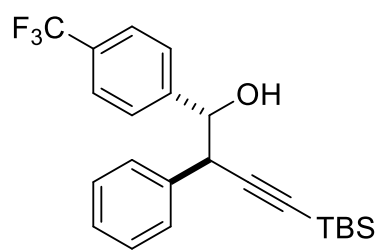

Yield: $55 \%, 89 \mathrm{mg}$

White solid, m.p. $=76.0-77.5^{\circ} \mathrm{C}$

$\mathrm{R}_{\mathrm{f}}\left(\mathrm{SiO}_{2}\right)=0.05($ Hexanes/ Ethyl acetate, $(20: 1))$.

${ }^{1} \mathrm{H}$ NMR $\left(300 \mathrm{MHz}, \mathrm{CDCl}_{3}\right) \delta 7.55(\mathrm{~d}, J=8.1 \mathrm{~Hz}, 2 \mathrm{H}), 7.38-7.26(\mathrm{~m}, 5 \mathrm{H}), 7.24-7.07(\mathrm{~m}, 2 \mathrm{H}), 4.94-$ $4.77(\mathrm{~m}, 1 \mathrm{H}), 4.01(\mathrm{~d}, J=6.0 \mathrm{~Hz}, 1 \mathrm{H}), 2.78(\mathrm{~d}, J=3.6 \mathrm{~Hz}, 1 \mathrm{H}), 0.98(\mathrm{~s}, 9 \mathrm{H}), 0.17(\mathrm{~s}, 6 \mathrm{H})$.

${ }^{13} \mathrm{C}$ NMR $\left(75 \mathrm{MHz}, \mathrm{CDCl}_{3}\right) \delta 145.0(\mathrm{C}), 137.2(\mathrm{C}), 128.9(4 \times \mathrm{CH}), 128.0(\mathrm{CH}), 127.4(2 \times \mathrm{CH})$, $125.2(\mathrm{~d}, J=3.4 \mathrm{~Hz}, 2 \times \mathrm{CH}), 104.6(\mathrm{C}), 90.0(\mathrm{C}), 77.8(\mathrm{CH}), 49.4(\mathrm{CH}), 26.5\left(3 \mathrm{x} \mathrm{CH}_{3}\right), 17.0(\mathrm{C})$, $4.2\left(2 \times \mathrm{CH}_{3}\right)$.

HRMS (ESI) m/z: [M + Na $]^{+}$Calcd. for $\mathrm{C}_{23} \mathrm{H}_{27} \mathrm{~F}_{3} \mathrm{NaOSi}$ : 427.1675; Found: 427.1682 . 


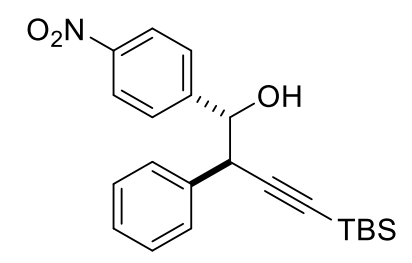

Yield: $42 \%, 64 \mathrm{mg}$

White solid, m.p. $=104.5-105.7^{\circ} \mathrm{C}$

$\mathrm{R}_{\mathrm{f}}\left(\mathrm{SiO}_{2}\right)=0.10$ (Hexanes/ Ethyl acetate, (10:1)).

${ }^{1} \mathrm{H}$ NMR $\left(300 \mathrm{MHz}, \mathrm{CDCl}_{3}\right) \delta 8.12(\mathrm{~d}, J=8.8 \mathrm{~Hz}, 2 \mathrm{H}), 7.36(\mathrm{~d}, J=8.7 \mathrm{~Hz}, 2 \mathrm{H}), 7.30(\mathrm{dt}, J=4.6$ and $2.2 \mathrm{~Hz}, 3 \mathrm{H}), 7.20(\mathrm{dd}, J=6.9,2.6 \mathrm{~Hz}, 2 \mathrm{H}), 4.93(\mathrm{dd}, J=5.8,3.2 \mathrm{~Hz}, 1 \mathrm{H}), 4.01(\mathrm{~d}, J=6.0 \mathrm{~Hz}$, $1 \mathrm{H}), 2.88(\mathrm{~d}, J=3.4 \mathrm{~Hz}, 1 \mathrm{H}), 0.97(\mathrm{~s}, 9 \mathrm{H}), 0.16(\mathrm{~s}, 6 \mathrm{H})$.

${ }^{13} \mathrm{C}$ NMR $\left(75 \mathrm{MHz}, \mathrm{CDCl}_{3}\right) \delta 147.9(\mathrm{C}), 147.4(\mathrm{C}), 136.4(\mathrm{C}), 128.6(2 \times \mathrm{CH}), 128.5(2 \times \mathrm{CH}), 127.8$ $(\mathrm{CH}), 127.5(2 \times \mathrm{CH}), 123.0(2 \times \mathrm{CH}), 103.9(\mathrm{C}), 89.8(\mathrm{C}), 77.1(\mathrm{CH}), 48.8(\mathrm{CH}), 26.1\left(3 \mathrm{x} \mathrm{CH}_{3}\right)$, $16.5(\mathrm{C}),-4.6\left(2 \times \mathrm{CH}_{3}\right)$.

HRMS (ESI) m/z: [M + Na] $]^{+}$Calcd. for $\mathrm{C}_{22} \mathrm{H}_{27} \mathrm{NNaO}_{3} \mathrm{Si}$ : 404.1652; Found: 404.1652 .

Anti-4-(tert-butyldimethylsilyl)-1-(3,5-dibromophenyl)-2-phenylbut-3-yn-1-ol (anti-5p)

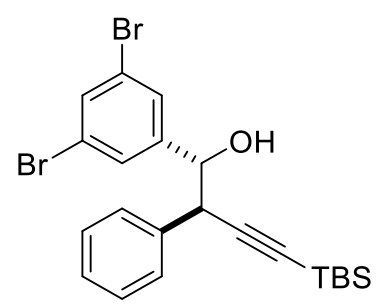

Yield: 54\%, $106 \mathrm{mg}$

Colorless oil

$\mathrm{R}_{\mathrm{f}}\left(\mathrm{SiO}_{2}\right)=0.07$ (Hexanes/ Ethyl acetate, (20:1)).

${ }^{1} \mathrm{H}$ NMR $\left(300 \mathrm{MHz}, \mathrm{CDCl}_{3}\right) \delta \delta 7.59(\mathrm{t}, J=1.7 \mathrm{~Hz}, 1 \mathrm{H}), 7.42-7.22(\mathrm{~m}, 7 \mathrm{H}), 4.75(\mathrm{~d}, J=3.6 \mathrm{~Hz}$, $1 \mathrm{H}), 3.98(\mathrm{~d}, J=5.1 \mathrm{~Hz}, 1 \mathrm{H}), 2.65(\mathrm{bs}, 1 \mathrm{H}), 0.99$ (s, 9H), 0.19 (s, 3H), 0.18 (s, 3H).

${ }^{13} \mathrm{C}$ NMR $\left(75 \mathrm{MHz}, \mathrm{CDCl}_{3}\right) \delta \delta 144.7(\mathrm{C}), 136.7(\mathrm{C}), 133.3(\mathrm{CH}), 128.6(2 \times \mathrm{CH}), 128.5(4 \times \mathrm{CH})$, $127.8(\mathrm{CH}), 122.4(2 \times \mathrm{C}), 103.4(\mathrm{C}), 90.1(\mathrm{C}), 76.4(\mathrm{CH}), 48.6(\mathrm{CH}), 26.1\left(3 \times \mathrm{CH}_{3}\right), 16.5(\mathrm{C}),-4.4$ $\left(\mathrm{CH}_{3}\right),-4.5\left(\mathrm{CH}_{3}\right)$.

HRMS (ESI) m/z: [M + H] ${ }^{+}$Calcd. for $\mathrm{C}_{22} \mathrm{H}_{27} \mathrm{Br}_{2} \mathrm{OSi}$ : 493.0192; Found: 493.0164 . 


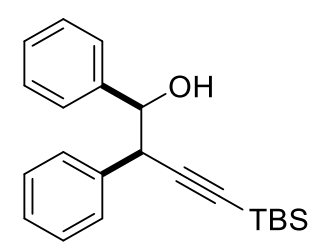

Yield: 47\%, $63 \mathrm{mg}$

Colorless oil

$\operatorname{Rf}\left(\mathrm{SiO}_{2}\right)=0.05($ Hexanes/ Ethyl acetate, $(20: 1))$.

${ }^{1} \mathrm{H}$ NMR $\left(300 \mathrm{MHz}, \mathrm{CDCl}_{3}\right) \delta=7.37-7.17(\mathrm{~m}, 10 \mathrm{H}), 4.83(\mathrm{dd}, J=7.1$ and $2.9 \mathrm{~Hz}, 1 \mathrm{H}), 4.04(\mathrm{~d}, J=$ $7.2 \mathrm{~Hz}, 1 \mathrm{H}), 2.31$ (d, J=3.2 Hz, 1H), $0.93(\mathrm{~s}, 9 \mathrm{H}), 0.11$ (s, 6H).

${ }^{13} \mathrm{C} \mathrm{NMR}\left(75 \mathrm{MHz}, \mathrm{CDCl}_{3}\right) \delta=140.8(\mathrm{C}), 137.8(\mathrm{C}), 129.0(2 \times \mathrm{CH}), 128.5(2 \times \mathrm{CH}), 128.0(\mathrm{CH})$, $127.9(2 \times \mathrm{CH}), 127.6(\mathrm{CH}), 127.2(2 \times \mathrm{CH}), 105.5(\mathrm{C}), 88.4(\mathrm{C}), 78.2(\mathrm{CH}), 48.3(\mathrm{CH}), 26.2(3 \times$ $\left.\mathrm{CH}_{3}\right), 16.6(\mathrm{C}),-4.5\left(2 \times \mathrm{CH}_{3}\right)$.

HRMS (ESI) m/z: [M + Na $]^{+}$Calcd. for $\mathrm{C}_{22} \mathrm{H}_{28} \mathrm{NaOSi}$ : 359.1802; Found: 359.1792 .

\section{Syn-4-(tert-butyldimethylsilyl)-2-phenyl-1-(p-tolyl)but-3-yn-1-ol (syn-5b)}

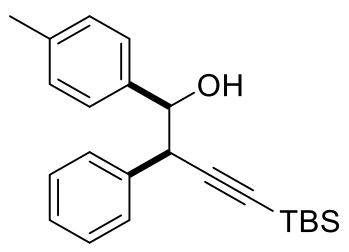

Yield: 45\%, $63 \mathrm{mg}$

White solid, m.p. $=78.6-80.0^{\circ} \mathrm{C}$

$\operatorname{Rf}(\mathrm{SiO} 2)=0.08($ Hexanes/ Ethyl acetate, $(20: 1))$.

${ }^{1} \mathrm{H}$ NMR $\left(300 \mathrm{MHz}, \mathrm{CDCl}_{3}\right) \delta 7.30(\mathrm{~d}, J=3.4 \mathrm{~Hz}, 5 \mathrm{H}), 7.16(\mathrm{~d}, J=8.1 \mathrm{~Hz}, 2 \mathrm{H}), 7.08(\mathrm{~d}, J=7.9 \mathrm{~Hz}$, 2H), $4.76(\mathrm{dd}, J=7.2$ and $3.5 \mathrm{~Hz}, 1 \mathrm{H}), 3.98(\mathrm{~d}, J=7.2 \mathrm{~Hz}, 1 \mathrm{H}), 2.33(\mathrm{~s}, 3 \mathrm{H}), 2.17(\mathrm{~d}, J=3.7 \mathrm{~Hz}$, $1 \mathrm{H}), 0.87(\mathrm{~s}, 9 \mathrm{H}), 0.05(\mathrm{~s}, 6 \mathrm{H})$.

${ }^{13} \mathrm{C} \mathrm{NMR}\left(75 \mathrm{MHz}, \mathrm{CDCl}_{3}\right) \delta=137.9(\mathrm{C}), 137.5(\mathrm{C}), 128.9(\mathrm{C}), 128.5(2 \times \mathrm{CH}), 128.4(2 \times \mathrm{CH})$, $127.4(\mathrm{CH}), 126.9(2 \times \mathrm{CH}), 105.5(\mathrm{C}), 88.1(\mathrm{C}), 78.0(\mathrm{CH}), 48.2(\mathrm{CH}), 26.1\left(3 \times \mathrm{CH}_{3}\right), 21.2\left(\mathrm{CH}_{3}\right)$, $16.5(\mathrm{C}),-4.6\left(2 \times \mathrm{CH}_{3}\right)$.

HRMS (ESI) m/z: [M + Na ${ }^{+}$Calcd. for $\mathrm{C}_{23} \mathrm{H}_{30} \mathrm{NaOSi}$ : 373.1958; Found: 359.1946. 


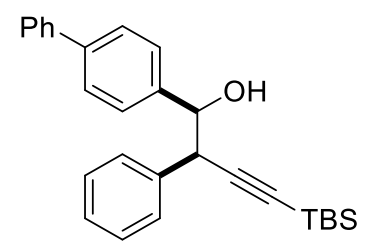

Yield: $44 \%, 71 \mathrm{mg}$

Colorless oil

$\mathrm{R}_{\mathrm{f}}\left(\mathrm{SiO}_{2}\right)=0.04($ Hexanes/ Ethyl acetate, $(20: 1))$.

${ }^{1} \mathrm{H}$ NMR $\left(300 \mathrm{MHz}, \mathrm{CDCl}_{3}\right) \delta=7.60(\mathrm{~d}, J=6.9 \mathrm{~Hz}, 2 \mathrm{H}), 7.54(\mathrm{~d}, J=8.2 \mathrm{~Hz}, 2 \mathrm{H}), 7.46(\mathrm{t}, J=7.4$ $\mathrm{Hz}, 2 \mathrm{H}), 7.35(\mathrm{td}, J=8.7,5.2 \mathrm{~Hz}, 8 \mathrm{H}), 4.95-4.73(\mathrm{~m}, 1 \mathrm{H}), 4.05(\mathrm{~d}, J=7.3 \mathrm{~Hz}, 1 \mathrm{H}), 2.28(\mathrm{~d}, J=3.5$ $\mathrm{Hz}, 1 \mathrm{H}), 0.89$ (s, 9H), 0.08 (s, 6H).

${ }^{13} \mathrm{C} \mathrm{NMR}\left(75 \mathrm{MHz}, \mathrm{CDCl}_{3}\right) \delta=141.1(\mathrm{C}), 140.9(\mathrm{C}), 139.9(\mathrm{C}), 137.8(\mathrm{C}), 129.0(2 \times \mathrm{CH}), 128.9(2$ x CH), $128.6(2 \times \mathrm{CH}), 127.7(\mathrm{CH}), 127.6(2 \times \mathrm{CH}), 127.4(\mathrm{CH}), 127.2(2 \times \mathrm{CH}), 126.7(2 \times \mathrm{CH})$, $105.5(\mathrm{C}), 88.5(\mathrm{C}), 78.1(\mathrm{CH}), 48.3(\mathrm{CH}), 26.2\left(3 \times \mathrm{CH}_{3}\right), 16.6(\mathrm{C}),-4.5\left(2 \mathrm{x} \mathrm{CH}_{3}\right)$.

HRMS (ESI) m/z: $[\mathrm{M}+\mathrm{Na}]^{+}$Calcd. for $\mathrm{C}_{28} \mathrm{H}_{32} \mathrm{NaOSi}$ : 435.2115; Found: 425.2114 .

\section{Syn-4-(tert-butyldimethylsilyl)-1-(4-chlorophenyl)-2-phenylbut-3-yn-1-ol (syn-5f)}

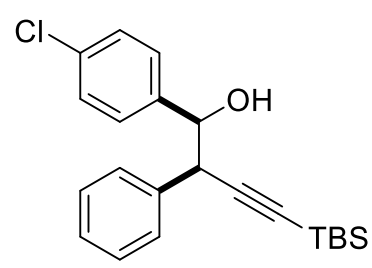

Yield: $47 \%, 70 \mathrm{mg}$

White solid, m.p. $=74.8-76.0{ }^{\circ} \mathrm{C}$

$\mathrm{R}_{\mathrm{f}}\left(\mathrm{SiO}_{2}\right)=0.07($ Hexanes/ Ethyl acetate, $(20: 1))$.

${ }^{1} \mathrm{H}$ NMR $\left(300 \mathrm{MHz}, \mathrm{CDCl}_{3}\right) \delta$ 7.45-7.25 (m, 7H), $7.21(\mathrm{~d}, J=8.5 \mathrm{~Hz}, 2 \mathrm{H}), 4.78(\mathrm{~d}, J=7.1 \mathrm{~Hz}, 1 \mathrm{H})$, $4.00(\mathrm{~d}, J=7.1 \mathrm{~Hz}, 1 \mathrm{H}), 2.34(\mathrm{~s}, 1 \mathrm{H}), 0.92(\mathrm{~s}, 9 \mathrm{H}), 0.10(\mathrm{~s}, 6 \mathrm{H})$.

${ }^{13} \mathrm{C} \mathrm{NMR}\left(75 \mathrm{MHz}, \mathrm{CDCl}_{3}\right) \delta 139.1(\mathrm{C}), 137.2(\mathrm{C}), 133.6(\mathrm{C}), 128.8(2 \times \mathrm{CH}), 128.5(2 \times \mathrm{CH}), 128.4$ $(2 \times \mathrm{CH}), 127.9(2 \times \mathrm{CH}), 127.6(\mathrm{CH}), 104.9(\mathrm{C}), 88.8(\mathrm{C}), 77.4(\mathrm{C}), 48.2(\mathrm{CH}), 26.0\left(3 \times \mathrm{CH}_{3}\right), 16.5$ (C), $-4.6\left(2 \times \mathrm{CH}_{3}\right)$.

HRMS (ESI) m/z: [M + Na $]^{+}$Calcd. for $\mathrm{C}_{22} \mathrm{H}_{27} \mathrm{NaOSi}$ : 393.1412; Found: 393.1405. 
Syn-1-(4-Bromophenyl)-4-(tert-butyldimethylsilyl)-2-phenylbut-3-yn-1-ol (syn-5g)

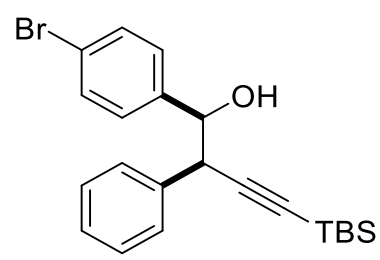

Yield: 46\%, $191 \mathrm{mg}$.

White solid, m.p. $=73.9-75.3^{\circ} \mathrm{C}$

$\mathrm{R}_{\mathrm{f}}\left(\mathrm{SiO}_{2}\right)=0.22($ Hexanes/ Ethyl acetate, (20:1)).

${ }^{1} \mathrm{H}$ NMR $\left(300 \mathrm{MHz}, \mathrm{CDCl}_{3}\right): \delta 7.40(\mathrm{~d}, J=8.3 \mathrm{~Hz}, 2 \mathrm{H}), 7.34-7.19(\mathrm{~m}, 5 \mathrm{H}), 7.13(\mathrm{~d}, J=8.3 \mathrm{~Hz}, 2 \mathrm{H})$, $4.74(\mathrm{~d}, J=6.8 \mathrm{~Hz}, 1 \mathrm{H}), 3.97(\mathrm{~d}, J=7.1 \mathrm{~Hz}, 1 \mathrm{H}), 2.30(\mathrm{~s}, 1 \mathrm{H}), 0.90(\mathrm{~s}, 9 \mathrm{H}), 0.08(\mathrm{~s}, 6 \mathrm{H})$.

${ }^{13} \mathrm{C}$ NMR $\left(75 \mathrm{MHz}, \mathrm{CDCl}_{3}\right): \delta 139.7(\mathrm{C}), 137.3(\mathrm{C}), 131.0(2 \times \mathrm{CH}), 128.9(2 \times \mathrm{CH}), 128.9(2 \times \mathrm{CH})$, $128.6(2 \times \mathrm{CH}), 127.8(\mathrm{CH}), 121.9(\mathrm{C}), 105.0(\mathrm{C}), 88.9(\mathrm{C}), 77.6(\mathrm{CH}), 48.2(\mathrm{CH}), 26.2\left(3 \times \mathrm{CH}_{3}\right)$, $16.6(\mathrm{C}),-4.5\left(2 \times \mathrm{CH}_{3}\right)$.

HRMS (ESI) m/z: [M + Na $]^{+}$Calcd. for $\mathrm{C}_{22} \mathrm{H}_{27} \mathrm{BrNaOSi}$ : 437.0912; Found: 437.0906.

Syn-4-(tert-butyldimethylsilyl)-1-(4-fluorophenyl)-2-phenylbut-3-yn-1-ol (syn-5h)

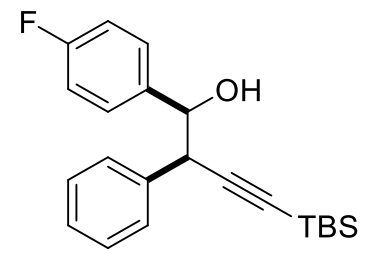

Yield: 44\%, $62 \mathrm{mg}$

White solid, m.p. $=66.3-67.3^{\circ} \mathrm{C}$

$\mathrm{R}_{\mathrm{f}}\left(\mathrm{SiO}_{2}\right)=0.08($ Hexanes/ Ethyl acetate, $(20: 1))$.

${ }^{1} \mathrm{H}$ NMR $\left(300 \mathrm{MHz}, \mathrm{CDCl}_{3}\right) \delta=7.44-7.10(\mathrm{~m}, 7 \mathrm{H}), 6.98(\mathrm{td}, J=9.0,2.3 \mathrm{~Hz}, 2 \mathrm{H}), 4.79(\mathrm{~d}, J=7.2$ $\mathrm{Hz}, 1 \mathrm{H}), 3.99$ (d, $J=7.1 \mathrm{~Hz}, 1 \mathrm{H}), 2.39$ (s, 1H), 0.92 (s, 9H), 0.10 (s, 6H).

${ }^{13} \mathrm{C} \mathrm{NMR}\left(75 \mathrm{MHz}, \mathrm{CDCl}_{3}\right) \delta=162.6(\mathrm{~d}, J=245.7 \mathrm{~Hz}, \mathrm{C}), 137.5(\mathrm{C}), 136.5(\mathrm{C}), 128.9(2 \times \mathrm{CH})$, $128.8(\mathrm{~d}, J=8.1 \mathrm{~Hz}, 2 \times \mathrm{CH}), 128.6(2 \times \mathrm{CH}), 127.7(\mathrm{CH}), 114.7(\mathrm{~d}, J=21.4 \mathrm{~Hz}, 2 \times \mathrm{CH}), 105.2$ (C), $88.7(\mathrm{C}), 77.6(\mathrm{CH}), 48.4(\mathrm{CH}), 26.2\left(3 \mathrm{x} \mathrm{CH}_{3}\right), 16.6(\mathrm{C}),-4.5\left(2 \times \mathrm{CH}_{3}\right)$.

${ }^{19} \mathrm{~F}$ NMR $\left(282 \mathrm{MHz}, \mathrm{CDCl}_{3}\right) \delta=-114.5$.

HRMS (ESI) m/z: [M + Na ${ }^{+}$Calcd. for $\mathrm{C}_{22} \mathrm{H}_{27} \mathrm{FNaOSi}$ : 377.1707; Found: 377.1721 . 


\section{General procedure for the synthesis of cis-2-silyl-4,5-dihydrofurans 3}
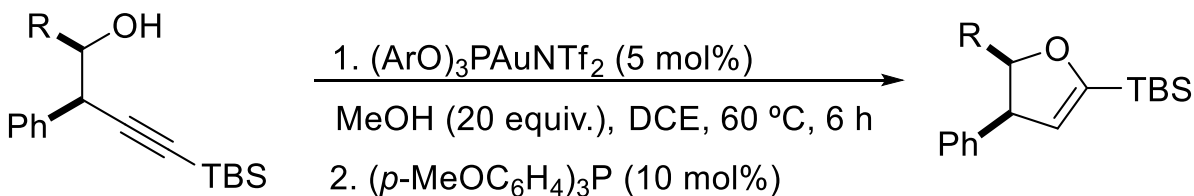

To a solution of $0.2 \mathrm{mmol}$ of the corresponding syn-homopropargyl alcohol in $1 \mathrm{~mL}$ dry $1,2-$ dichloroethane at were added $161 \mu \mathrm{L}(128 \mathrm{mg} ; 4 \mathrm{mmol} ; 20$ equivalents) of methanol followed by $11.2 \mathrm{mg}$ of the gold catalyst $(5 \mathrm{~mol} \%)$. The reaction was heated, in an oil bath, at $60{ }^{\circ} \mathrm{C}$ for 6 hours. Then, $8.2 \mathrm{mg}(0.02 \mathrm{mmol} ; 10 \mathrm{~mol} \%)$ of $\left(p-\mathrm{MeO}-\mathrm{C}_{6} \mathrm{H}_{4}\right)_{3} \mathrm{P}$ was added. After cooling down, solvents were removed under vacuum. The residue was purified under deactivated aluminium oxide flash chromatography to obtain the corresponding cis-2-silyl-4,5-dihydrofurans 3 .

\section{Cis-tert-butyldimethyl-4,5-diphenyl-4,5-dihydrofuran-2-yl)dimethylsilane (3a)}

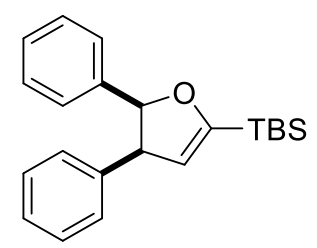

Yield: 99\%, $67 \mathrm{mg}$

Colorless oil

$\mathrm{R}_{\mathrm{f}}\left(\mathrm{SiO}_{2}\right)=0.22($ Hexanes/ Ethyl acetate, $(200: 1))$.

${ }^{1} \mathrm{H}$ NMR $\left(300 \mathrm{MHz}, \mathrm{CDCl}_{3}\right) \delta=7.11-6.94(\mathrm{~m}, 8 \mathrm{H}), 6.92-6.83(\mathrm{~m}, 2 \mathrm{H}), 5.79(\mathrm{~d}, J=10.1 \mathrm{~Hz}, 1 \mathrm{H})$, $5.46(\mathrm{~d}, J=2.7 \mathrm{~Hz}, 1 \mathrm{H}), 4.30(\mathrm{dd}, J=10.1$ and $2.7 \mathrm{~Hz}, 1 \mathrm{H}), 1.08(\mathrm{~s}, 9 \mathrm{H}), 0.27(\mathrm{~s}, 3 \mathrm{H}), 0.24(\mathrm{~s}, 3 \mathrm{H})$.

${ }^{13} \mathrm{C}$ NMR $\left(75 \mathrm{MHz}, \mathrm{CDCl}_{3}\right) \delta=162.7(\mathrm{C}), 139.8(\mathrm{C}), 138.7(\mathrm{C}), 129.1(2 \times \mathrm{CH}), 127.7(2 \times \mathrm{CH})$, $127.5(2 \times \mathrm{CH}), 126.7(3 \times \mathrm{CH}), 126.3(\mathrm{CH}), 117.6(\mathrm{CH}), 88.1(\mathrm{CH}), 54.6(\mathrm{CH}), 26.8\left(3 \times \mathrm{CH}_{3}\right), 16.7$ (C), $-6.2\left(\mathrm{CH}_{3}\right),-6.3\left(\mathrm{CH}_{3}\right)$.

HRMS (ESI) m/z: $[\mathrm{M}+\mathrm{H}]^{+}$Calcd. for $\mathrm{C}_{22} \mathrm{H}_{29} \mathrm{OSi}$ : 337.2139; Found: 337.2127. 


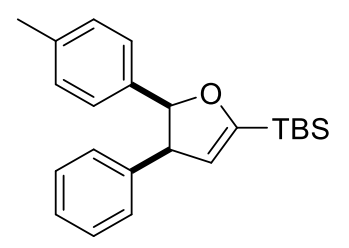

Yield: 99\%, $69 \mathrm{mg}$

Colorless oil

$\mathrm{R}_{\mathrm{f}}\left(\mathrm{SiO}_{2}\right)=0.24$ (Hexanes/ Ethyl acetate, (200:1)).

${ }^{1} \mathrm{H}$ NMR $\left(300 \mathrm{MHz}, \mathrm{CDCl}_{3}\right) \delta=7.39-7.30(\mathrm{~m}, 2 \mathrm{H}), 7.30-7.13(\mathrm{~m}, 7 \mathrm{H}), 5.27(\mathrm{~d}, J=2.5 \mathrm{~Hz}, 1 \mathrm{H}), 5.26$ $(\mathrm{d}, J=7.1 \mathrm{~Hz}, 1 \mathrm{H}), 4.01(\mathrm{dd}, J=7.1$ and $2.5 \mathrm{~Hz}, 1 \mathrm{H}), 2.36(\mathrm{~s}, 3 \mathrm{H}), 1.04(\mathrm{~s}, 9 \mathrm{H}), 0.23(\mathrm{~s}, 3 \mathrm{H}), 0.21$ (s, $3 \mathrm{H})$.

${ }^{13} \mathrm{C}$ NMR $\left(75 \mathrm{MHz}, \mathrm{CDCl}_{3}\right) \delta=161.9(\mathrm{C}), 144.6(\mathrm{C}), 140.6(\mathrm{C}), 137.3(\mathrm{C}), 129.3(2 \times \mathrm{CH}), 128.8(2$ x CH), $127.7(2 \times \mathrm{CH}), 126.8(\mathrm{CH}), 125.5(2 \times \mathrm{CH}), 116.2(\mathrm{CH}), 92.0(\mathrm{CH}), 59.5(\mathrm{CH}), 26.7(3 \times$ $\left.\mathrm{CH}_{3}\right), 21.3\left(\mathrm{CH}_{3}\right), 16.7(\mathrm{C}),-6.3\left(\mathrm{CH}_{3}\right),-6.3\left(\mathrm{CH}_{3}\right)$.

RMS (ESI) m/z: $[\mathrm{M}+\mathrm{H}]^{+}$Calcd. for $\mathrm{C}_{23} \mathrm{H}_{31} \mathrm{OSi}$ : 351.2139; Found: 351.2127 .

\section{Cis-(5-([1,1'-biphenyl]-4-yl)-4-phenyl-4,5-dihydrofuran-2-yl)(tert-butyl)dimethylsilane} (3d)

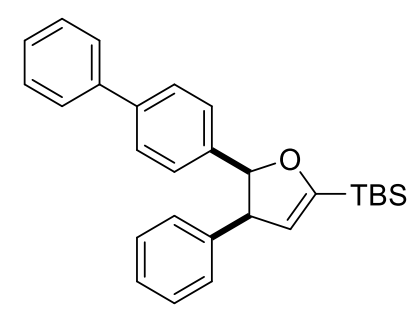

Yield: $96 \%, 79 \mathrm{mg}$

Colorless oil

$\mathrm{R}_{\mathrm{f}}\left(\mathrm{SiO}_{2}\right)=0.16$ (Hexanes/ Ethyl acetate, (200:1)).

${ }^{1} \mathrm{H}$ NMR $\left(300 \mathrm{MHz}, \mathrm{CDCl}_{3}\right) \delta=7.56-7.44(\mathrm{~m}, 2 \mathrm{H}), 7.44 .-28(\mathrm{~m}, 5 \mathrm{H}), 7.15-88(\mathrm{~m}, 7 \mathrm{H}), 5.82(\mathrm{~d}, J=$ $10.0 \mathrm{~Hz}, 1 \mathrm{H}), 5.48(\mathrm{~d}, J=2.7 \mathrm{~Hz}, 1 \mathrm{H}), 4.33(\mathrm{dd}, J=10.0$ and $2.7 \mathrm{~Hz}, 1 \mathrm{H}), 1.10(\mathrm{~s}, 9 \mathrm{H}), 0.29(\mathrm{~s}, 3 \mathrm{H})$, $0.26(\mathrm{~s}, 3 \mathrm{H})$.

${ }^{13} \mathrm{C}$ NMR $\left(75 \mathrm{MHz}, \mathrm{CDCl}_{3}\right) \delta=162.7$ (C), $141.1(\mathrm{C}), 139.7$ (C), 139.4 (C), 137.9 (C), $129.1(2 \mathrm{x}$ $\mathrm{CH}), 128.7(2 \times \mathrm{CH}), 127.8(2 \times \mathrm{CH}), 127.2(2 \times \mathrm{CH}), 127.1(\mathrm{CH}), 127.0(2 \times \mathrm{CH}), 126.4(\mathrm{CH})$, $126.2(2 \times \mathrm{CH}), 117.6(\mathrm{CH}), 88.0(\mathrm{CH}), 54.6(\mathrm{CH}), 26.8\left(3 \times \mathrm{CH}_{3}\right), 16.7(\mathrm{C}),-6.2\left(\mathrm{CH}_{3}\right),-6.3\left(\mathrm{CH}_{3}\right)$.

HRMS (ESI) m/z: [M + H] $]^{+}$Calcd. for $\mathrm{C}_{28} \mathrm{H}_{33} \mathrm{OSi}$ : 413.2295; Found: 413.2301. 


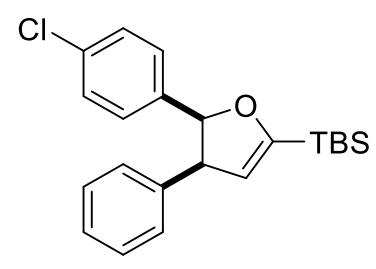

Yield: $98 \%, 73 \mathrm{mg}$

Colorless oil

$\mathrm{R}_{\mathrm{f}}\left(\mathrm{SiO}_{2}\right)=0.20($ Hexanes/ Ethyl acetate, $(200: 1))$.

${ }^{1} \mathrm{H}$ NMR $\left(300 \mathrm{MHz}, \mathrm{CDCl}_{3}\right) \delta=7.11-7.00(\mathrm{~m}, 5 \mathrm{H}), 6.99-6.91(\mathrm{~m}, 2 \mathrm{H}), 6.89-6.82(\mathrm{~m}, 2 \mathrm{H}), 5.73(\mathrm{dd}$, $J=10.1$ and $0.8 \mathrm{~Hz}, 1 \mathrm{H}), 5.45(\mathrm{dd}, J=2.7$ and $0.6 \mathrm{~Hz}, 1 \mathrm{H}), 4.29(\mathrm{dd}, J=10.1$ and $2.7 \mathrm{~Hz}, 1 \mathrm{H}), 1.07$ $(\mathrm{s}, 9 \mathrm{H}), 0.26(\mathrm{~s}, 3 \mathrm{H}), 0.24(\mathrm{~s}, 3 \mathrm{H})$.

${ }^{13} \mathrm{C} \mathrm{NMR}\left(75 \mathrm{MHz}, \mathrm{CDCl}_{3}\right) \delta=162.6(\mathrm{C}), 139.4(\mathrm{C}), 137.3(\mathrm{C}), 132.4(\mathrm{C}), 129.0(2 \times \mathrm{CH}), 128.1(2$ x CH), $127.9(2 \times \mathrm{CH}), 127.7(2 \times \mathrm{CH}), 126.6(\mathrm{CH}), 117.6(\mathrm{CH}), 87.4(\mathrm{CH}), 54.5(\mathrm{CH}), 26.8(3 \mathrm{x}$ $\left.\mathrm{CH}_{3}\right), 16.7(\mathrm{C}),-6.3\left(\mathrm{CH}_{3}\right),-6.3\left(\mathrm{CH}_{3}\right)$.

HRMS (ESI) m/z: $[\mathrm{M}+\mathrm{H}]^{+}$Calcd. for $\mathrm{C}_{22} \mathrm{H}_{28} \mathrm{ClOSi}$ : 371.1592; Found: 371.1595 .

Cis-tert-butyldimethyl(5-(p-bromophenyl)-4-phenyl-4,5-dihydrofuran-2-yl)silane (3g)

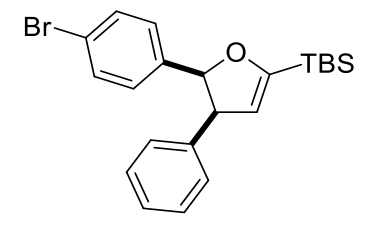

Yield: $99 \%, 82 \mathrm{mg}$.

Colorless oil

$\mathrm{R}_{\mathrm{f}}\left(\mathrm{SiO}_{2}\right)=0.21$ (Hexanes/ Ethyl acetate, (200:1)).

${ }^{1} \mathrm{H}$ NMR $\left(300 \mathrm{MHz}, \mathrm{CDCl}_{3}\right) \delta(\mathrm{ppm}) 7.21(\mathrm{~d}, J=8.5 \mathrm{~Hz}, 2 \mathrm{H}), 7.14-7.00(\mathrm{~m}, 3 \mathrm{H}), 6.99-6.81(\mathrm{~m}, 4 \mathrm{H})$, $5.73(\mathrm{~d}, J=10.1 \mathrm{~Hz}, 1 \mathrm{H}), 5.46(\mathrm{dd}, J=2.7$ and $0.5 \mathrm{~Hz}, 1 \mathrm{H}), 2 \mathrm{H}), 4.30(\mathrm{dd}, J=10.1$ and $2.7 \mathrm{~Hz}$, $1 \mathrm{H}), 1.08(\mathrm{~s}, 9 \mathrm{H}), 0.27(\mathrm{~s}, 3 \mathrm{H}), 0.25(\mathrm{~s}, 3 \mathrm{H})$.

${ }^{13} \mathrm{C} \mathrm{NMR}\left(75 \mathrm{MHz}, \mathrm{CDCl}_{3}\right) \delta 162.6(\mathrm{C}), 139.4(\mathrm{C}), 137.8(\mathrm{C}), 130.6(2 \times \mathrm{CH}), 129.0(2 \times \mathrm{CH})$, $128.5(2 \times \mathrm{CH}), 127.9(2 \times \mathrm{CH}), 126.7(\mathrm{CH}), 120.5(\mathrm{C}), 117.7(\mathrm{CH}), 87.4(\mathrm{CH}), 54.4(\mathrm{CH}), 26.8(3 \times$ $\left.\mathrm{CH}_{3}\right), 16.7(\mathrm{C}),-6.3\left(\mathrm{CH}_{3}\right),-6.3\left(\mathrm{CH}_{3}\right)$.

HRMS (ESI) m/z: [M + H] ${ }^{+}$Calcd. for $\mathrm{C}_{22} \mathrm{H}_{28}$ BrOSi: Calc.: 415.1093; Found: 415.1087. 


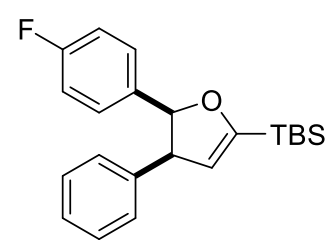

Yield: $98 \%, 69 \mathrm{mg}$

Colorless oil

$\mathrm{R}_{\mathrm{f}}\left(\mathrm{SiO}_{2}\right)=0.31$ (Hexanes/ Ethyl acetate, (200:1)).

${ }^{1} \mathrm{H}$ NMR (300 MHz, $\left.\mathrm{CDCl}_{3}\right) \delta=7.13-6.96(\mathrm{~m}, 5 \mathrm{H}), 6.92-6.85(\mathrm{~m}, 2 \mathrm{H}), 6.83-6.71(\mathrm{~m}, 2 \mathrm{H}), 5.78(\mathrm{~d}, J$ $=10.1 \mathrm{~Hz}, 1 \mathrm{H}), 5.47(\mathrm{~d}, J=2.7,1 \mathrm{H}), 4.30(\mathrm{dd}, J=10.1,2.7 \mathrm{~Hz}, 1 \mathrm{H}), 1.10(\mathrm{~s}, 9 \mathrm{H}), 0.29(\mathrm{~s}, 3 \mathrm{H}), 0.26$ (s, 3H).

${ }^{13} \mathrm{C} \mathrm{NMR}\left(75 \mathrm{MHz}, \mathrm{CDCl}_{3}\right) \delta=162.7(\mathrm{C}), 161.7(\mathrm{~d}, J=244.4 \mathrm{~Hz}, \mathrm{C}), 139.6(\mathrm{C}), 134.5(\mathrm{~d}, J=3.2$ $\mathrm{Hz}, \mathrm{C}), 129.0(2 \times \mathrm{CH}), 128.3(\mathrm{~d}, J=3.2 \mathrm{~Hz}, 2 \mathrm{x} \mathrm{CH}), 127.9(2 \times \mathrm{CH}), 126.5(\mathrm{CH}), 117.5(\mathrm{CH})$, $114.4(\mathrm{~d}, J=21.3 \mathrm{~Hz}, 2 \times \mathrm{CH}), 87.5(\mathrm{CH}), 54.5(\mathrm{CH}), 26.8\left(3 \times \mathrm{CH}_{3}\right), 16.7(\mathrm{C}),-6.3\left(\mathrm{CH}_{3}\right),-6.3$ $\left(\mathrm{CH}_{3}\right)$.

${ }^{19} \mathrm{~F} \mathrm{NMR}\left(282 \mathrm{MHz}, \mathrm{CDCl}_{3}\right) \delta=-116.0$.

HRMS (ESI) m/z: $[\mathrm{M}+\mathrm{H}]^{+}$Calcd. for $\mathrm{C}_{22} \mathrm{H}_{28} \mathrm{FOSi}$ : 355.1888; Found: 355.1889 . 


\section{NMR Spectra}

Trans-tert-butyldimethyl-4,5-diphenyl-4,5-dihydrofuran-2-yl)dimethylsilane (4a)

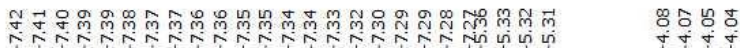

${ }^{1} \mathrm{H}-\mathrm{NMR}\left(300 \mathrm{MHz} ; \mathrm{CDCl}_{3}\right)$<smiles>CC(C)(C)[13C]1=C[C@@H](c2ccccc2)O[C@H]1c1ccccc1</smiles>

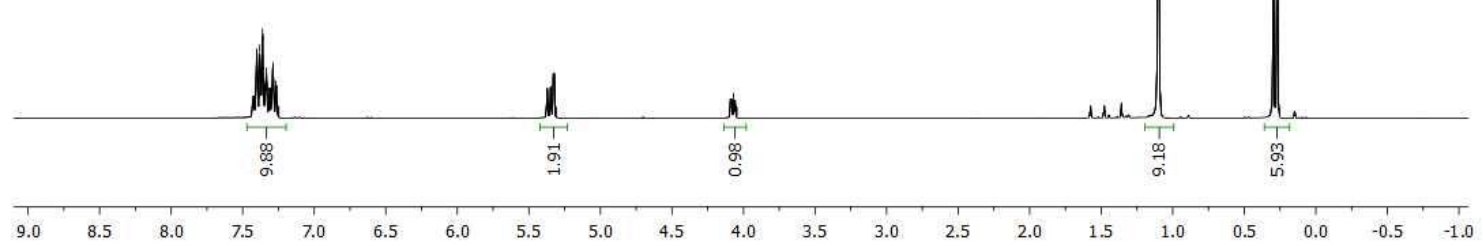

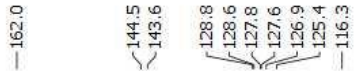

${ }^{13} \mathrm{C}-\mathrm{NMR}\left(75 \mathrm{MHz} ; \mathrm{CDCl}_{3}\right)$

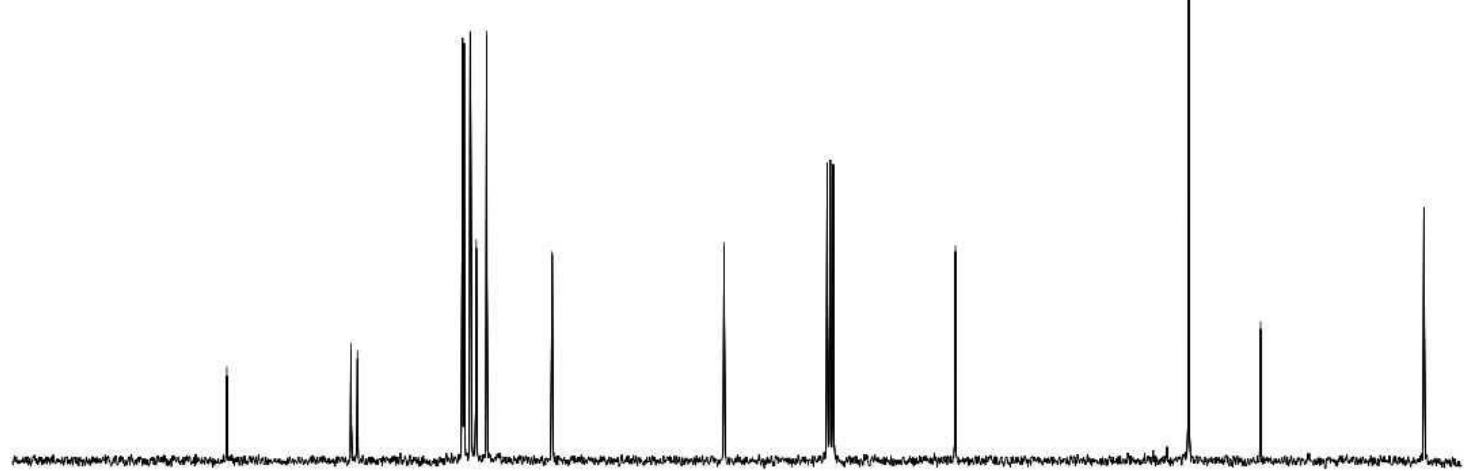

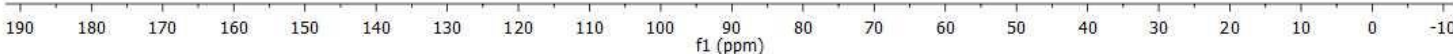


Trans-tert-butyldimethyl(4-phenyl-5-( $p$-tolyl)-4,5-dihydrofuran-2-yl)silane (4b)

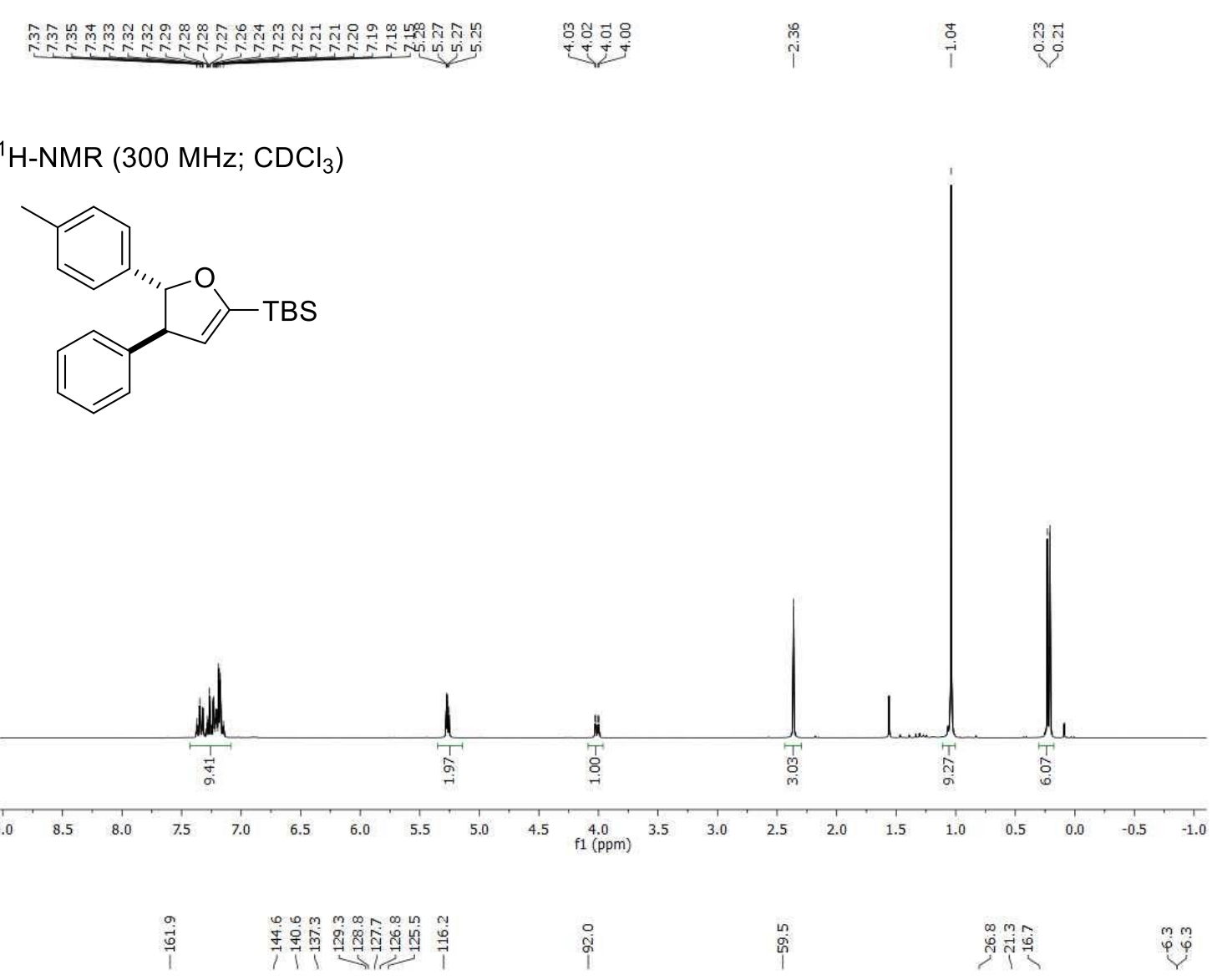

${ }^{13} \mathrm{C}-\mathrm{NMR}\left(75 \mathrm{MHz} ; \mathrm{CDCl}_{3}\right.$ )

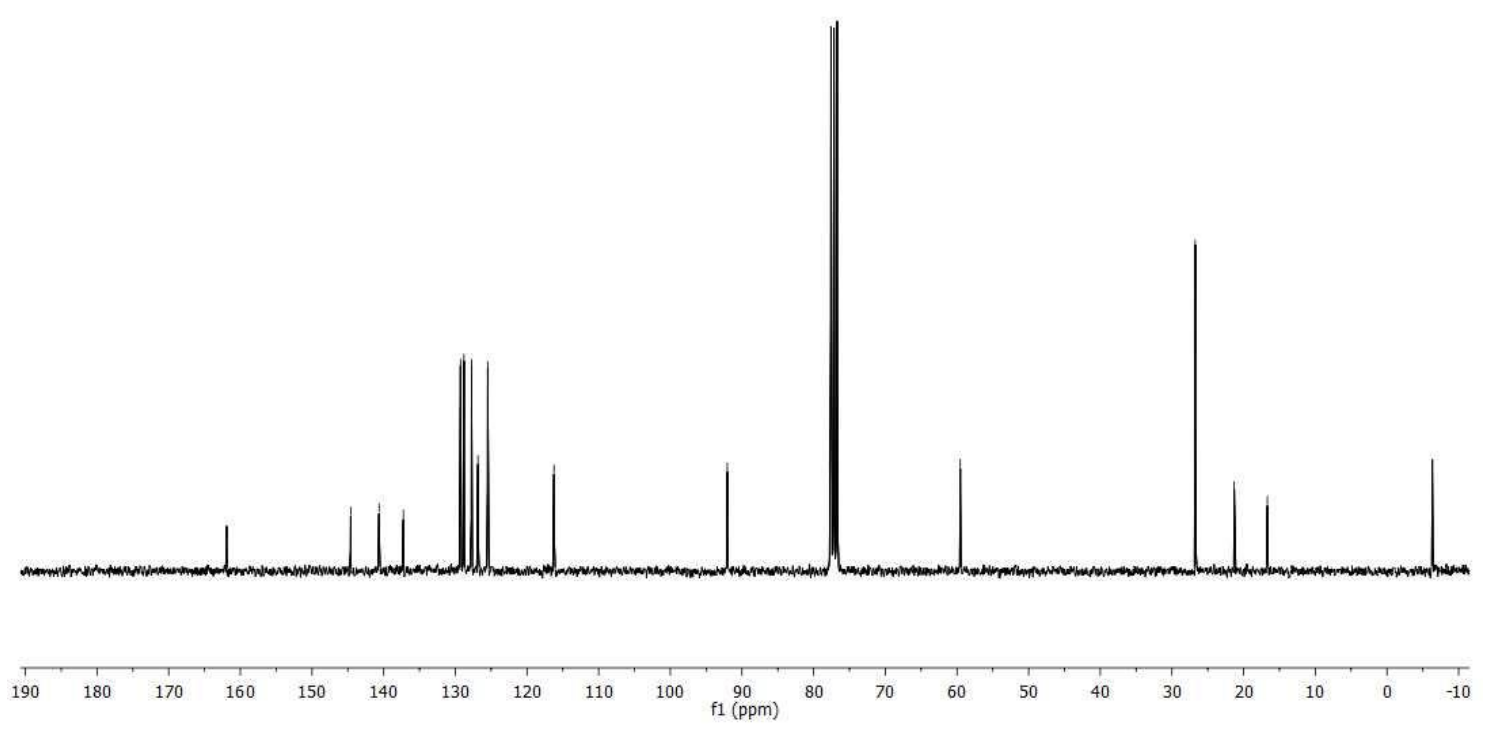


Trans-tert-butyl(5-(4-(tert-butyl)phenyl)-4-phenyl-4,5-dihydrofuran-2-yl)dimethylsilane (4c).

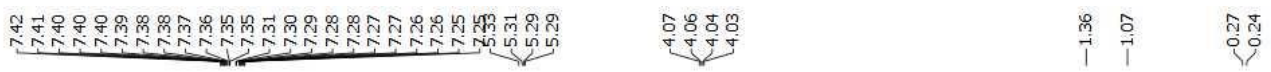

${ }^{1} \mathrm{H}-\mathrm{NMR}\left(300 \mathrm{MHz} ; \mathrm{CDCl}_{3}\right)$<smiles>CC(C)(C)c1ccc(C2OC([135I])=CC2c2ccccc2)cc1</smiles>

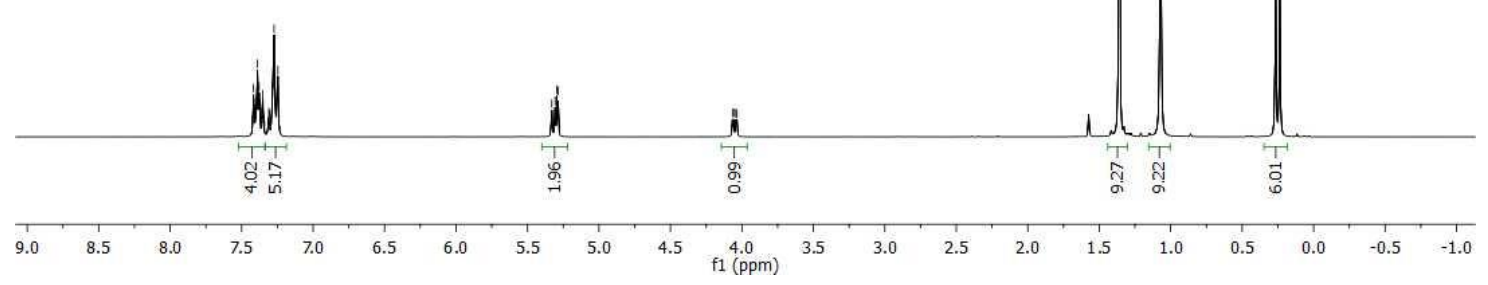

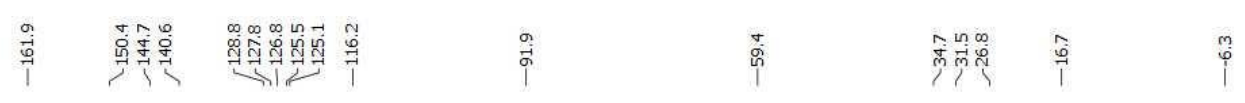

${ }^{13} \mathrm{C}-\mathrm{NMR}\left(75 \mathrm{MHz} ; \mathrm{CDCl}_{3}\right)$

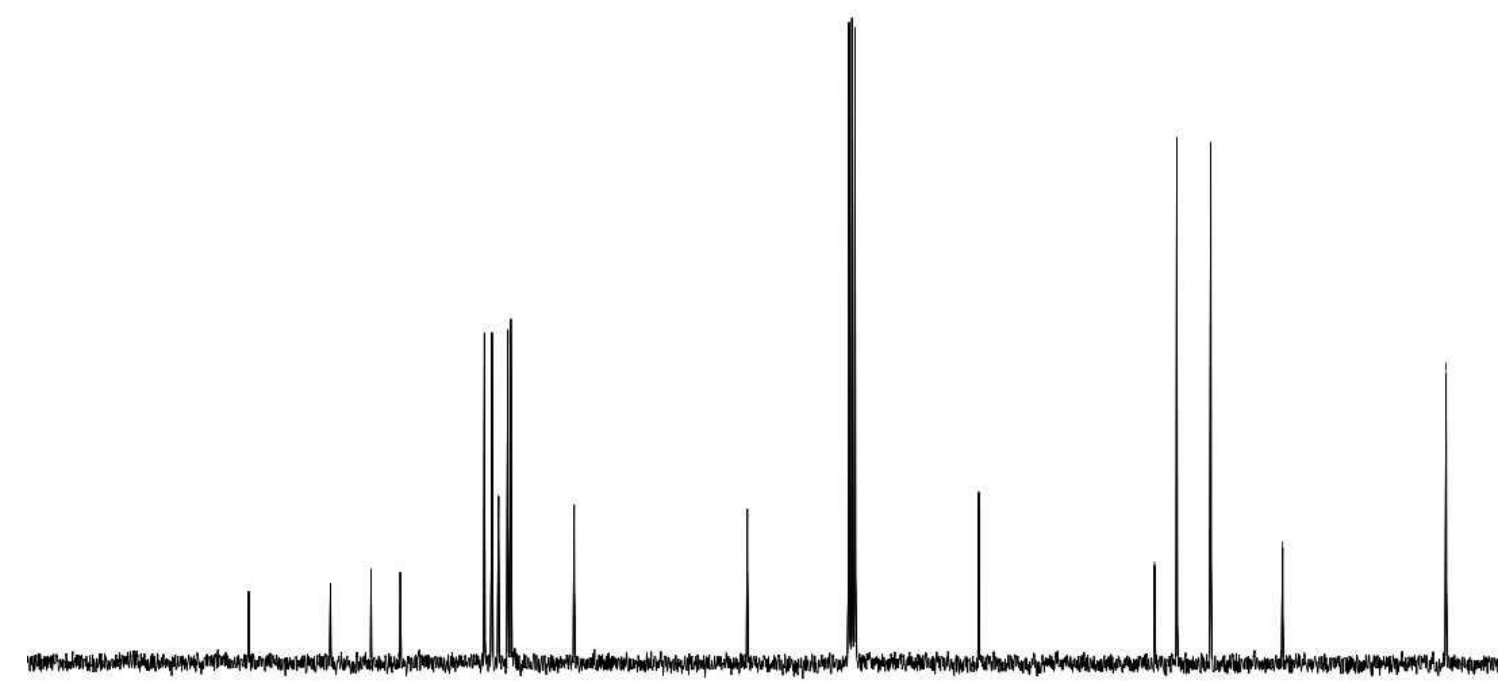

\begin{tabular}{llllllllllllllllllllllll}
\hline & 190 & 180 & 170 & 160 & 150 & 140 & 130 & 120 & 110 & 100 & 90 & 80 & 70 & 60 & 50 & 40 & 30 & 20 & 10 & 0 & -10
\end{tabular} 
Trans-(5-([1,1'-biphenyl]-4-yl)-4-phenyl-4,5-dihydrofuran-2-yl)(tertbutyl)dimethylsilane (4d).

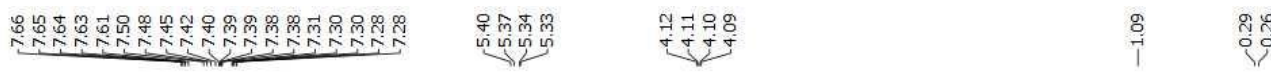

${ }^{1} \mathrm{H}-\mathrm{NMR}\left(300 \mathrm{MHz} ; \mathrm{CDCl}_{3}\right)$<smiles>CC(C)(C)C1=C[C@@H](c2ccccc2)[C@H](c2ccc(-c3ccccc3)cc2)O1</smiles>

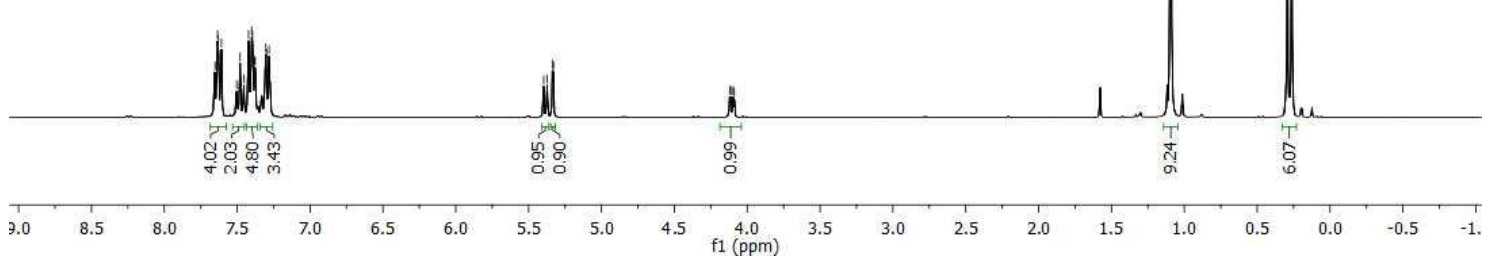

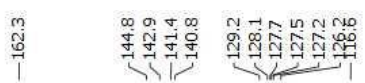

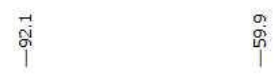

${ }^{13} \mathrm{C}-\mathrm{NMR}\left(75 \mathrm{MHz} ; \mathrm{CDCl}_{3}\right)$

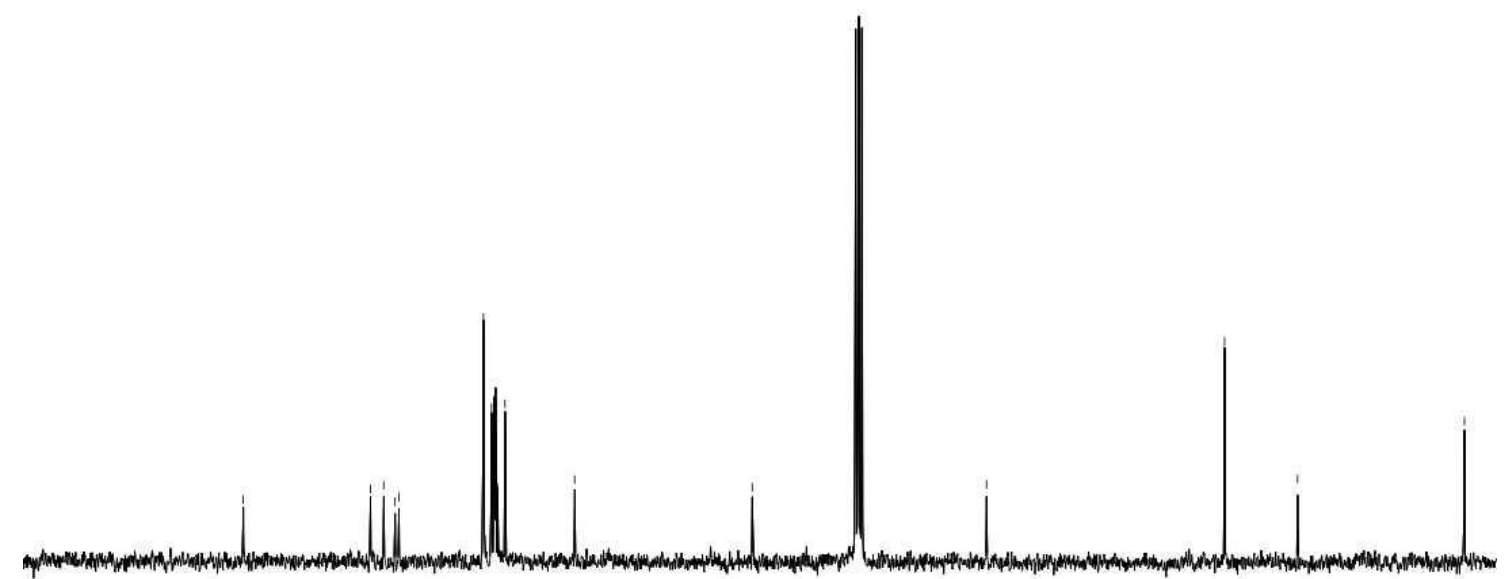

$\begin{array}{lllllllllllllllllllllll}190 & 180 & 170 & 160 & 150 & 140 & 130 & 120 & 110 & 100 & 90 & 80 & 70 & 60 & 50 & 40 & 30 & 20 & 10 & 0 & -1\end{array}$ 
Trans-4-(5-(tert-butyldimethylsilyl)-3-phenyl-2,3-dihydrofuran-2-yl)phenyl benzoate (4e).
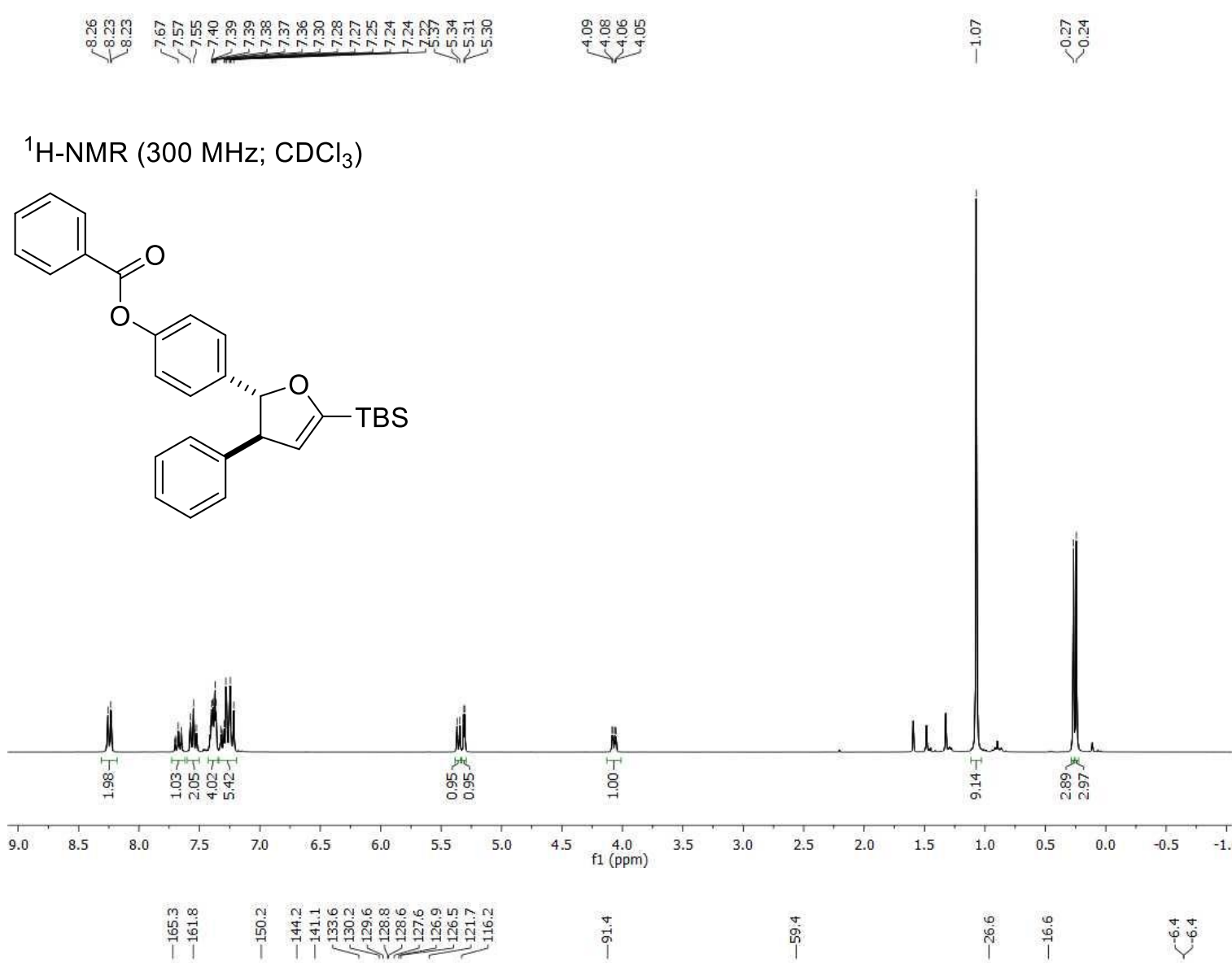

${ }^{13} \mathrm{C}-\mathrm{NMR}\left(75 \mathrm{MHz} ; \mathrm{CDCl}_{3}\right.$ )

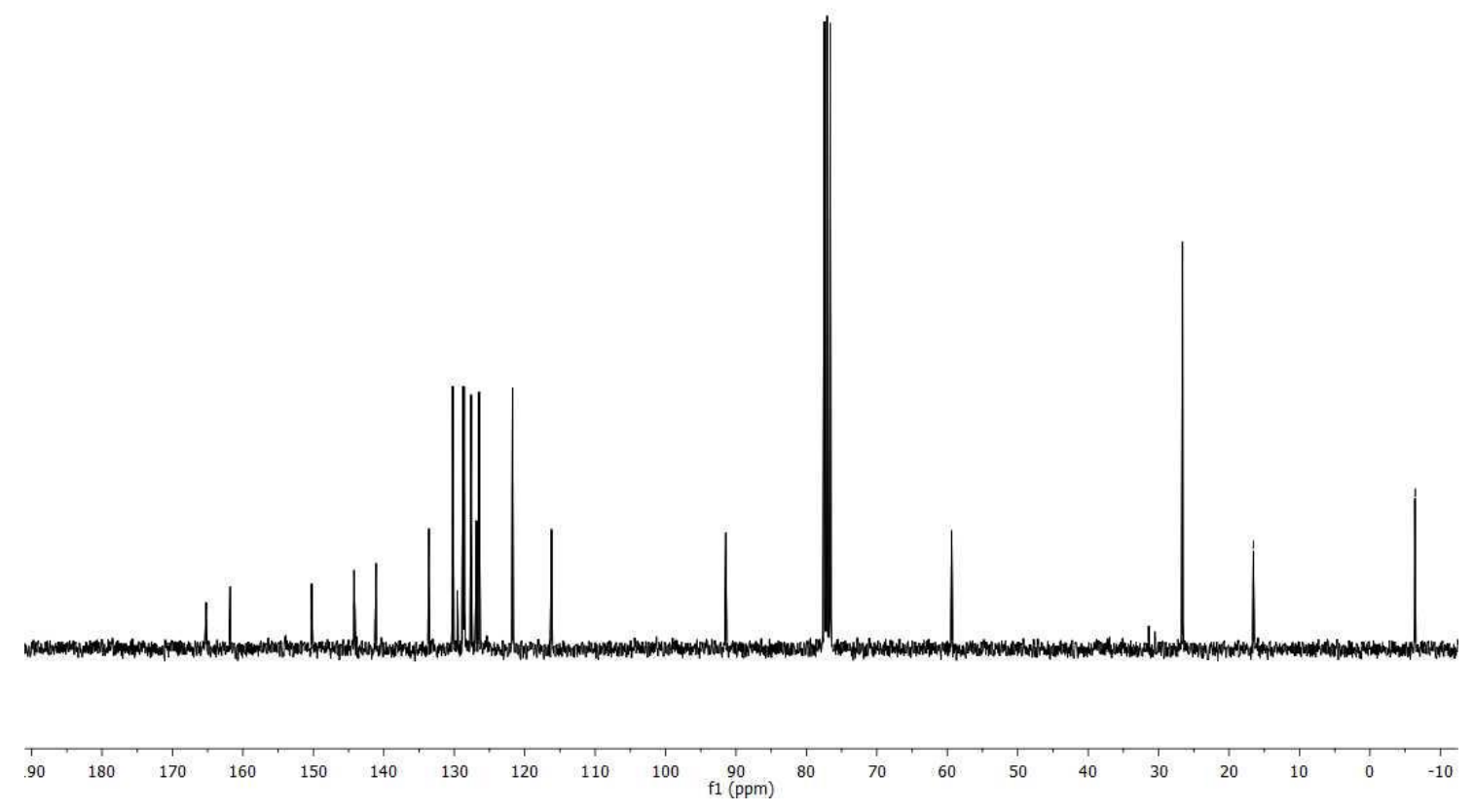




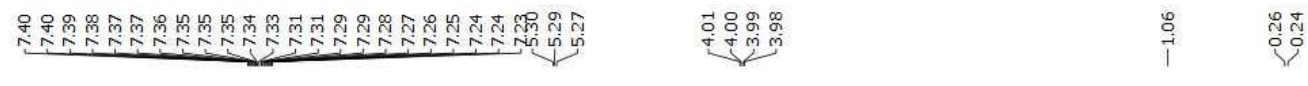

${ }^{1} \mathrm{H}-\mathrm{NMR}\left(300 \mathrm{MHz} ; \mathrm{CDCl}_{3}\right)$

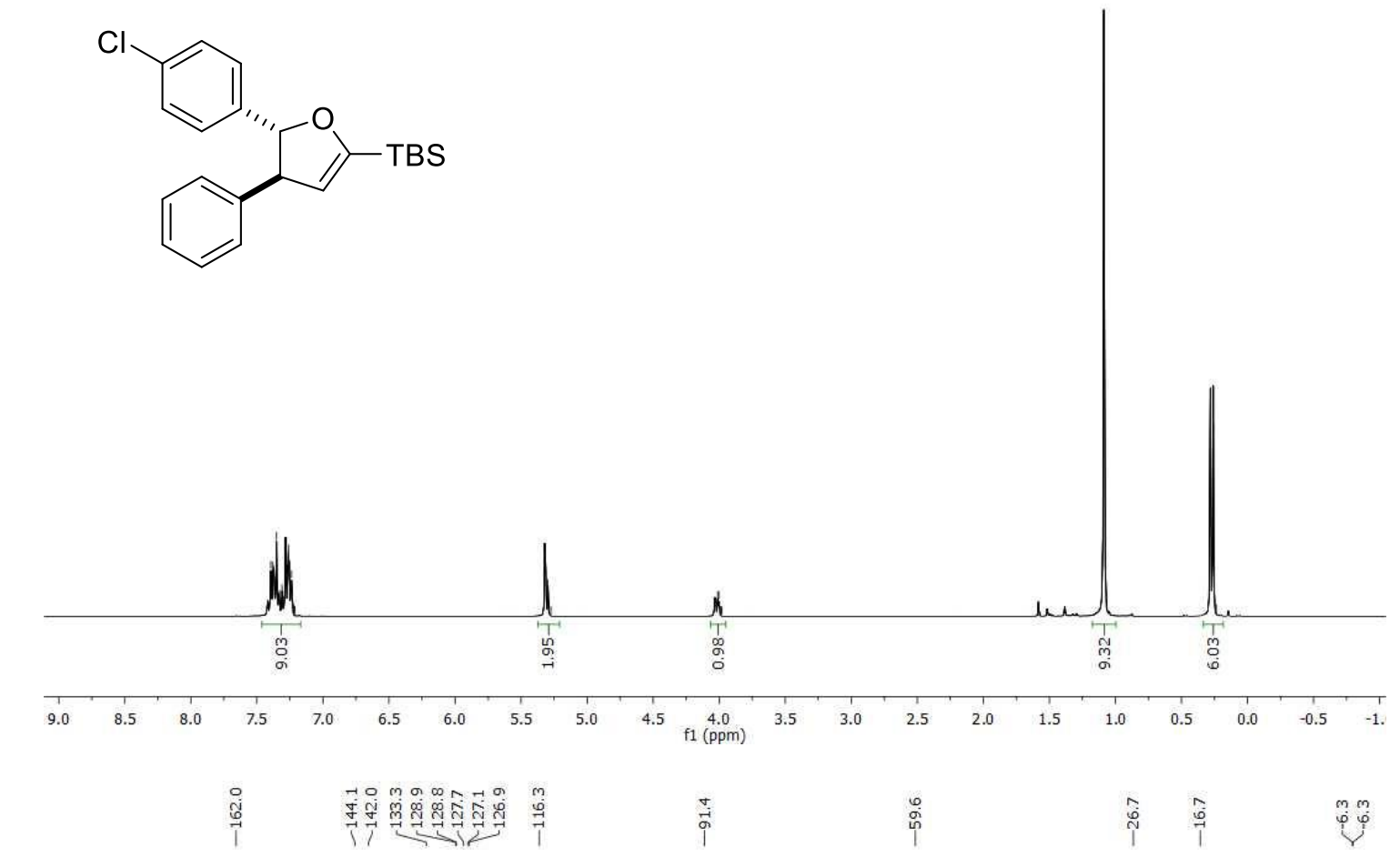

${ }^{13} \mathrm{C}-\mathrm{NMR}\left(75 \mathrm{MHz} ; \mathrm{CDCl}_{3}\right)$

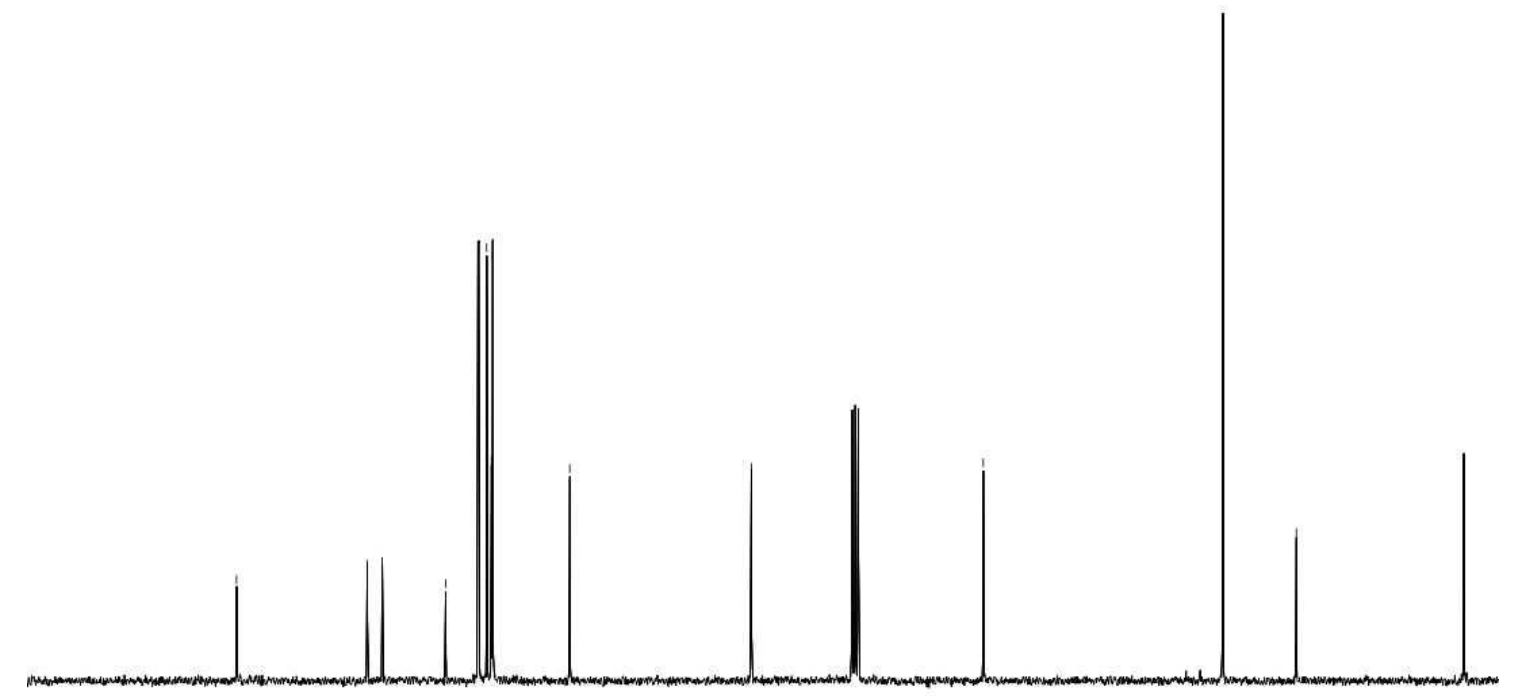

\begin{tabular}{lllllllllllllllllllll}
\hline & 180 & 170 & 160 & 150 & 140 & 130 & 120 & 110 & 100 & 90 & 80 & 70 & 60 & 50 & 40 & 30 & 20 & 10 & 0 & -10
\end{tabular} 
Trans-tert-butyldimethyl(5-(p-bromophenyl)-4-phenyl-4,5-dihydrofuran-2-yl)silane (4g).

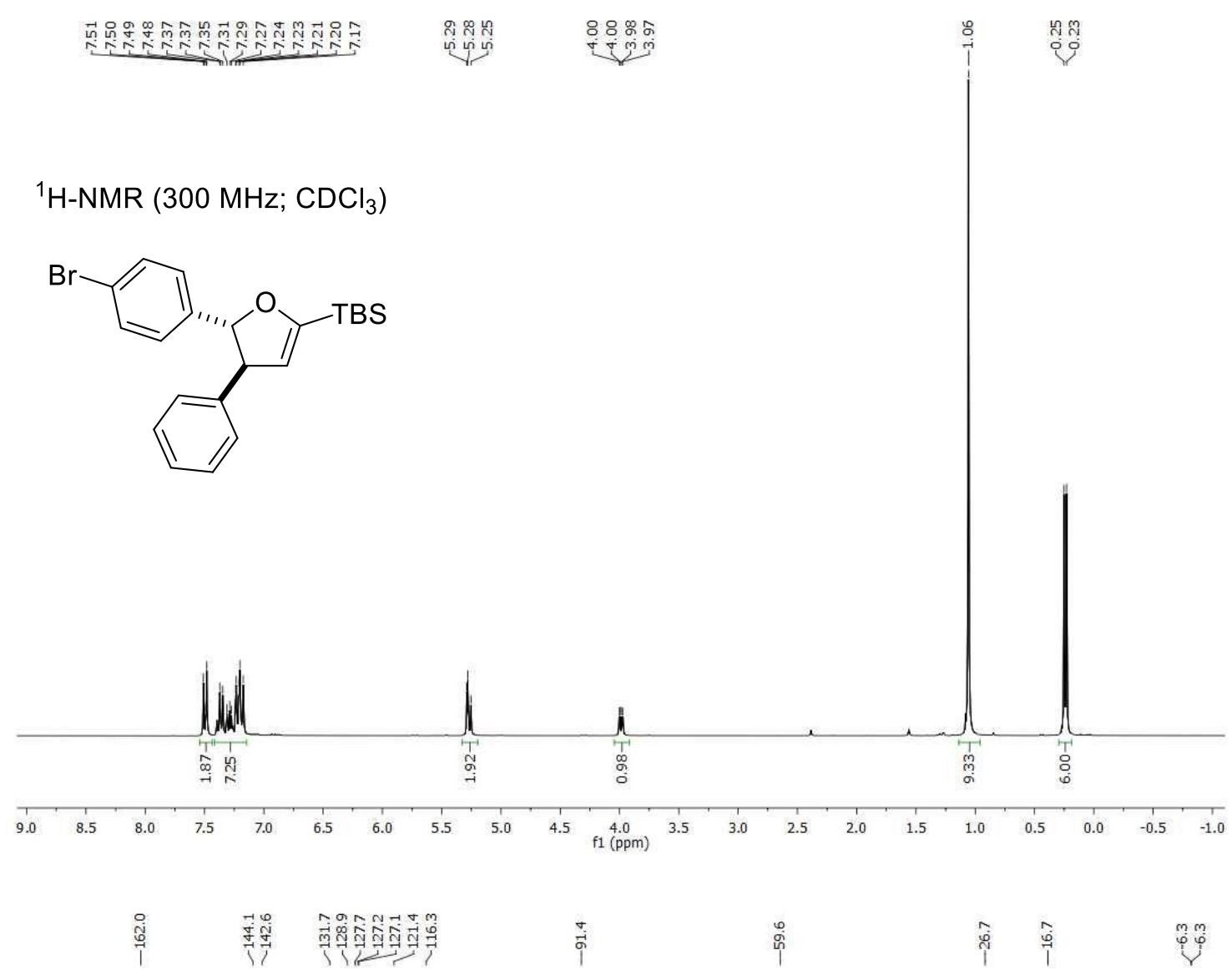

${ }^{13} \mathrm{C}-\mathrm{NMR}\left(75 \mathrm{MHz} ; \mathrm{CDCl}_{3}\right)$

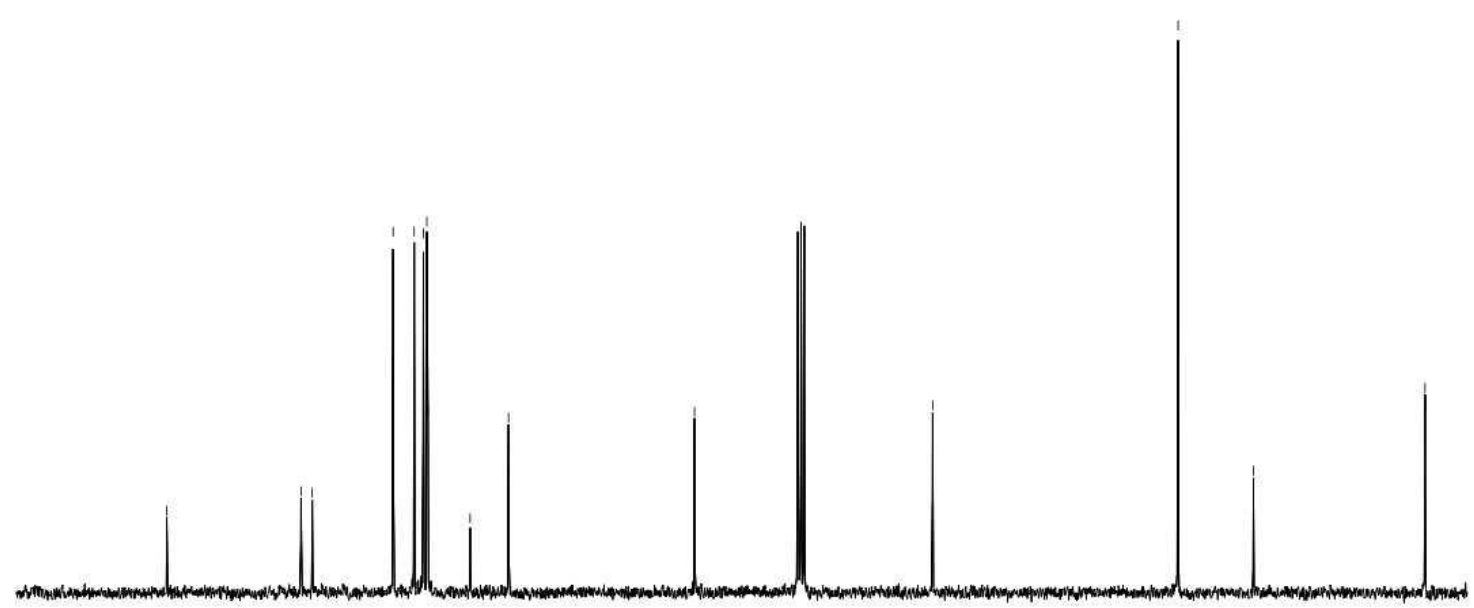

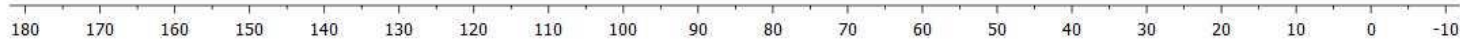


Trans-tert-butyl(5-(4-fluorophenyl)-4-phenyl-4,5-dihydrofuran-2-yl)dimethylsilane (4h).
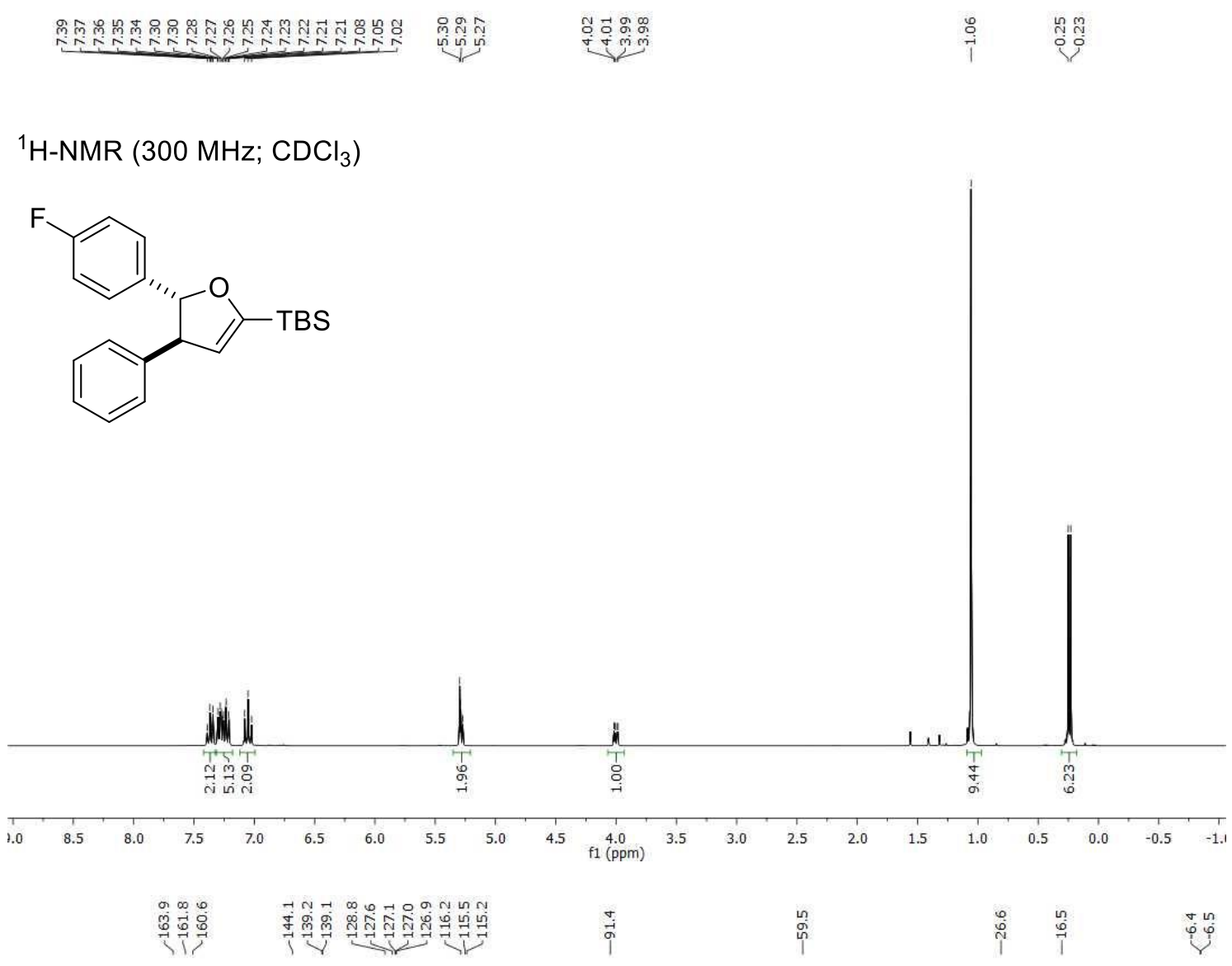

${ }^{13} \mathrm{C}-\mathrm{NMR}\left(75 \mathrm{MHz} ; \mathrm{CDCl}_{3}\right)$

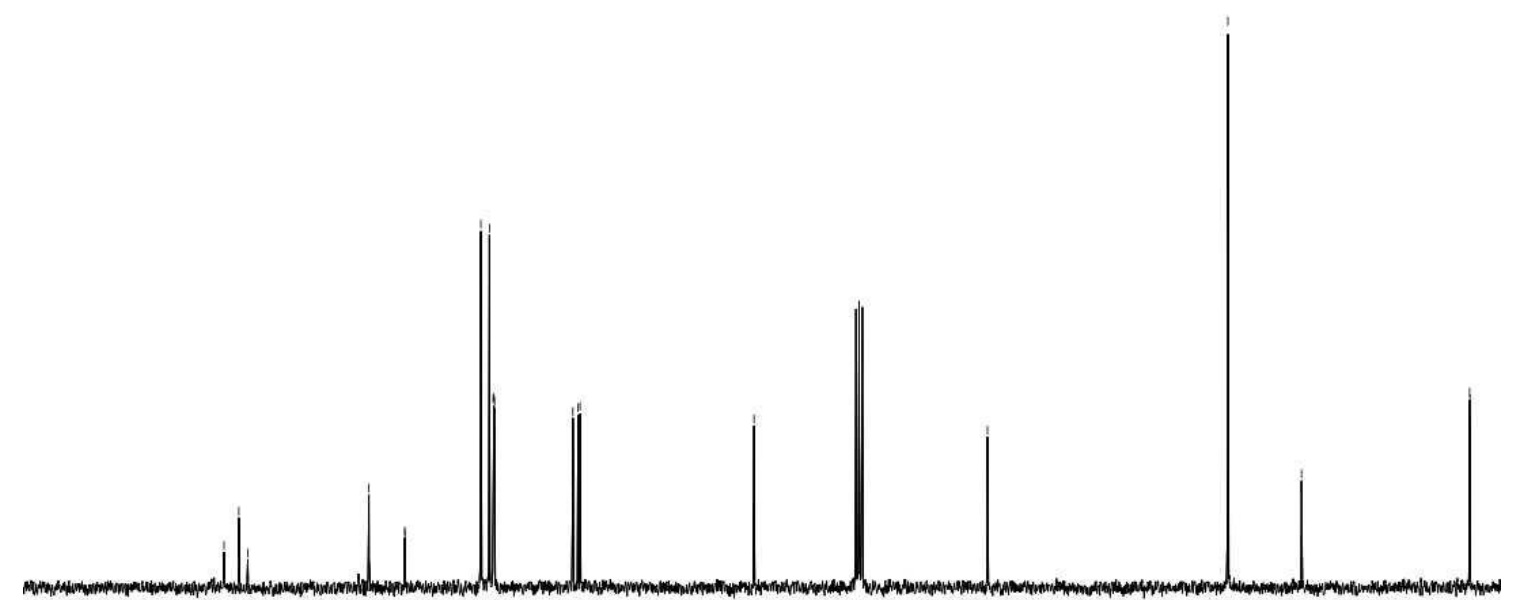

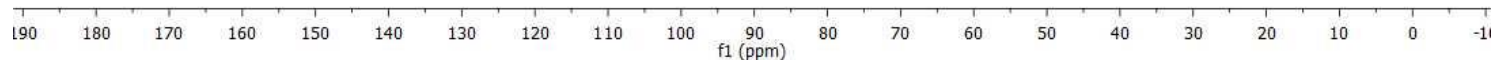


Methyl 4-(trans-5-(tert-butyldimethylsilyl)-3-phenyl-2,3-dihydrofuran-2-yl)benzoate (4i).

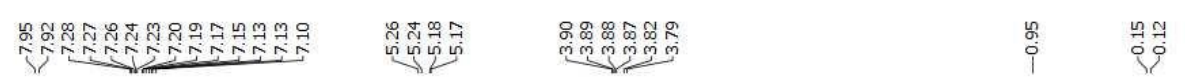

${ }^{1} \mathrm{H}-\mathrm{NMR}\left(300 \mathrm{MHz} ; \mathrm{CDCl}_{3}\right.$ )<smiles>COC(=O)c1ccc([C@@H]2OC([AsH3-])=C[C@H]2c2ccccc2)cc1</smiles>

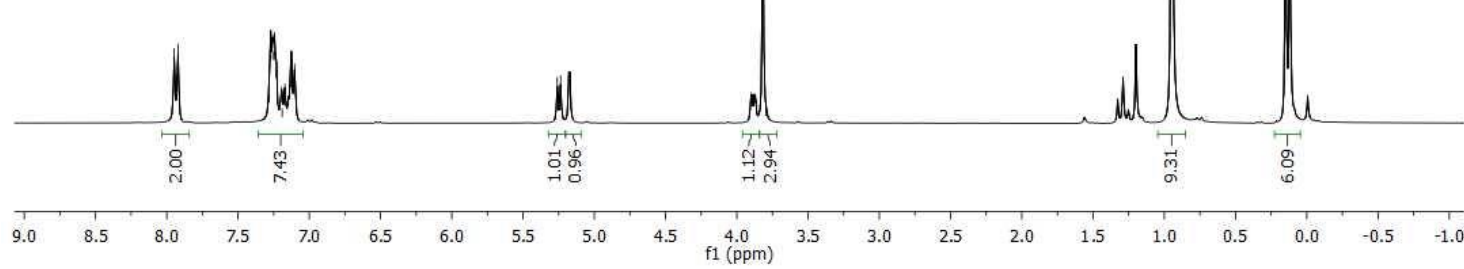

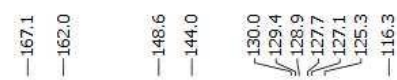

$\stackrel{n}{\rightarrow}$

ฺั กี่

${ }^{13} \mathrm{C}-\mathrm{NMR}\left(75 \mathrm{MHz} ; \mathrm{CDCl}_{3}\right.$ )

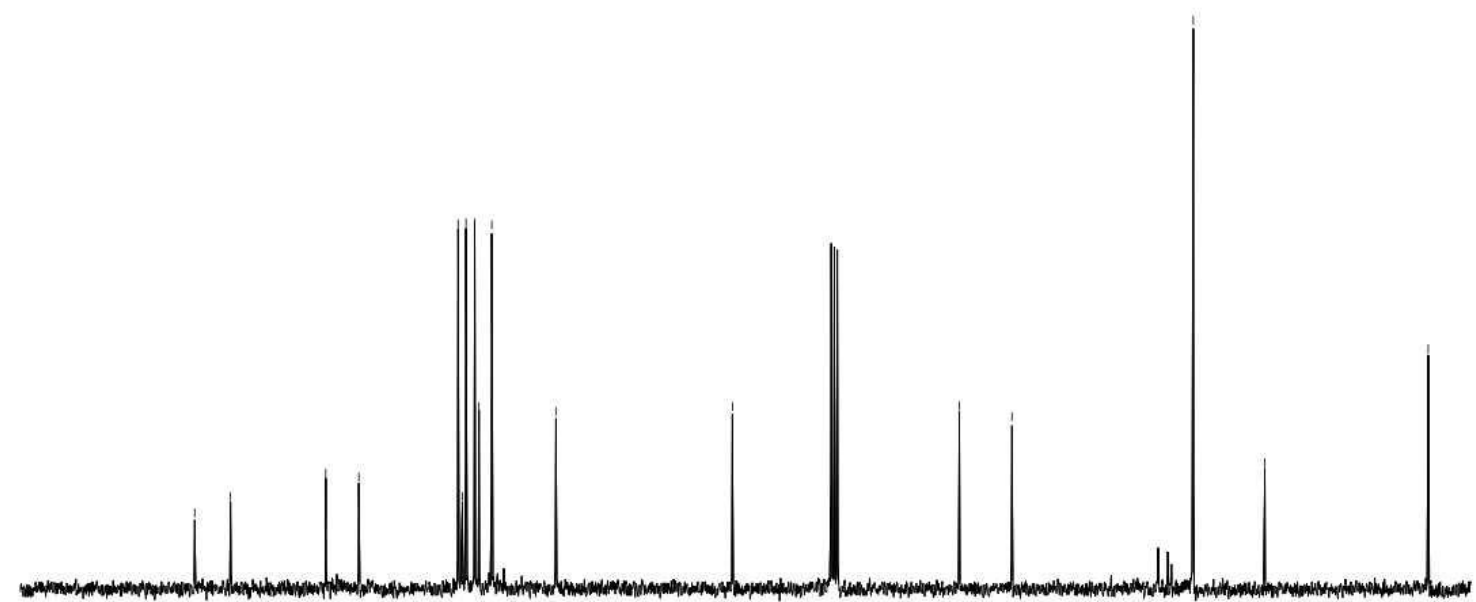

$\begin{array}{llllllllll}190 & 180 & 170 & 160 & 150 & 140 & 130 & 120 & 110 & 10\end{array}$ 
Trans-tert-butyldimethyl(5-(4-nitrophenyl)-4-phenyl-4,5-dihydrofuran-2-yl)silane (4k).

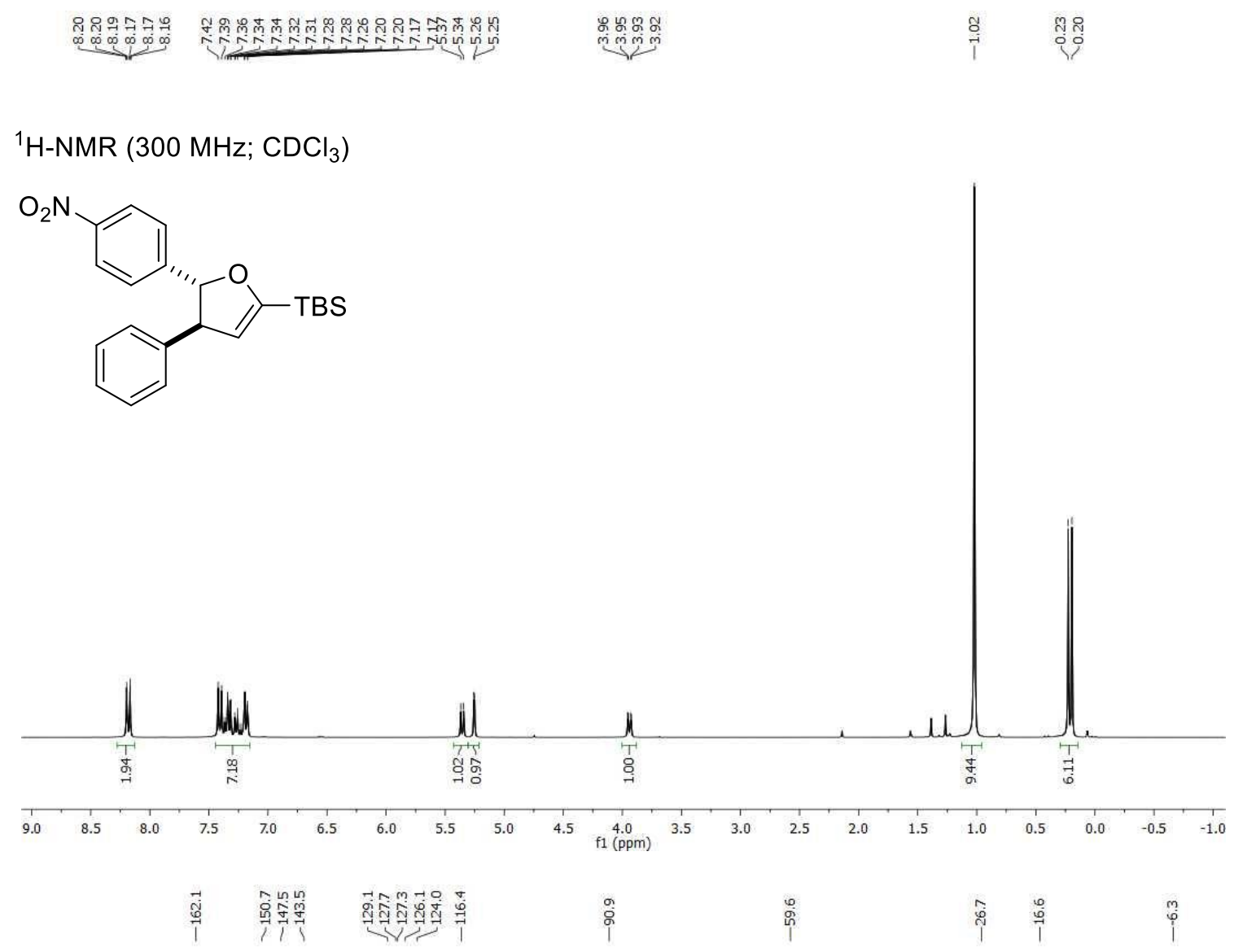

${ }^{13} \mathrm{C}-\mathrm{NMR}\left(75 \mathrm{MHz} ; \mathrm{CDCl}_{3}\right.$ )

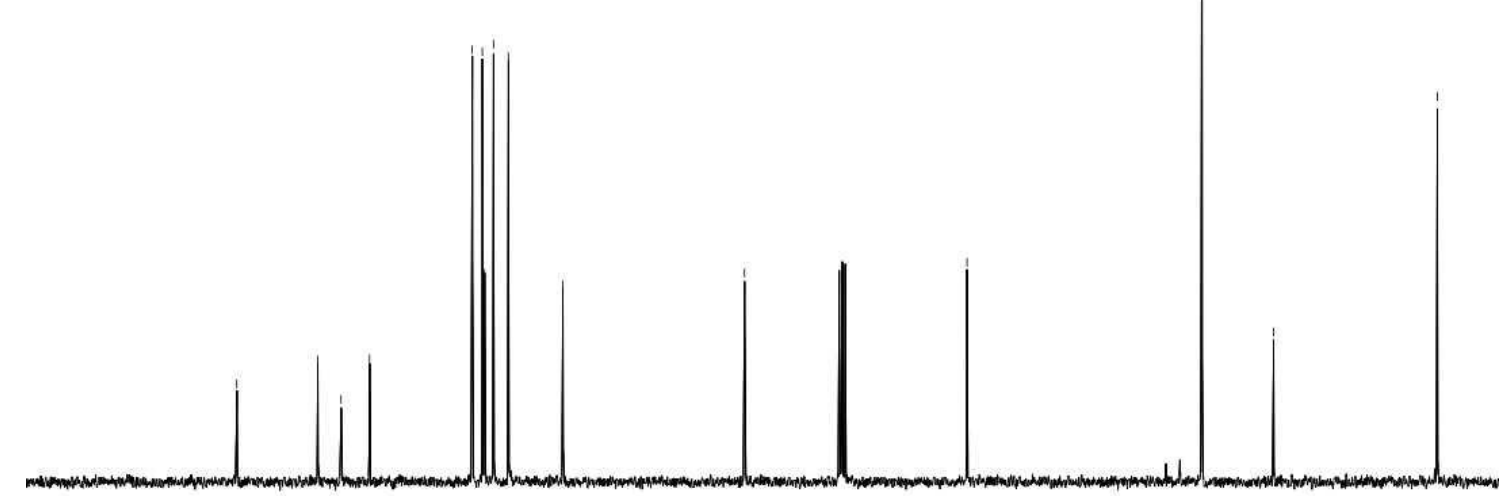


Trans-tert-butyldimethyl(4-phenyl-5-(m-tolyl)-4,5-dihydrofuran-2-yl)silane (4l).

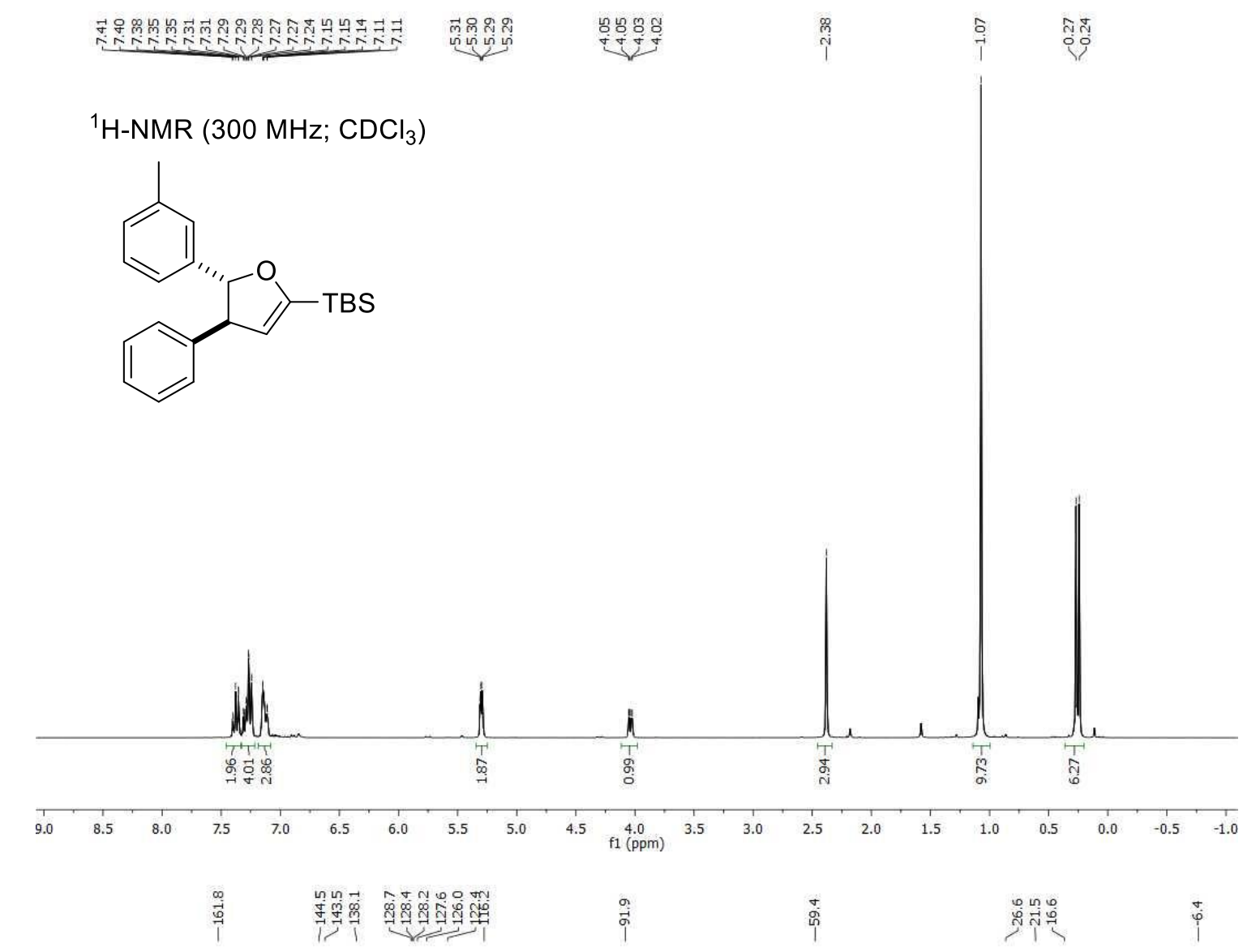

${ }^{13} \mathrm{C}-\mathrm{NMR}\left(75 \mathrm{MHz} ; \mathrm{CDCl}_{3}\right)$

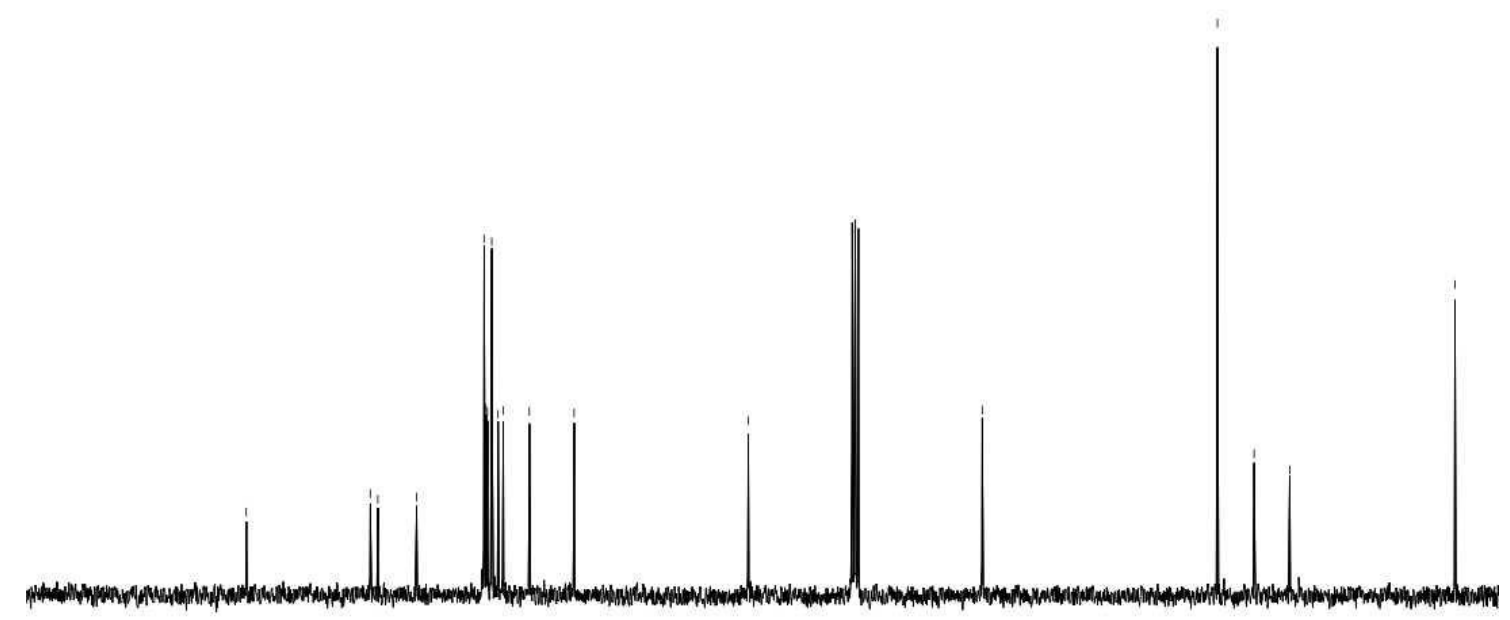

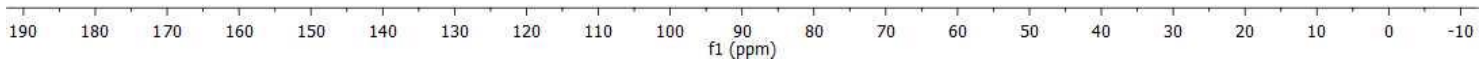


Trans-tert-butyl(5-(3-methoxyphenyl)-4-phenyl-4,5-dihydrofuran-2-yl)dimethylsilane (4m).

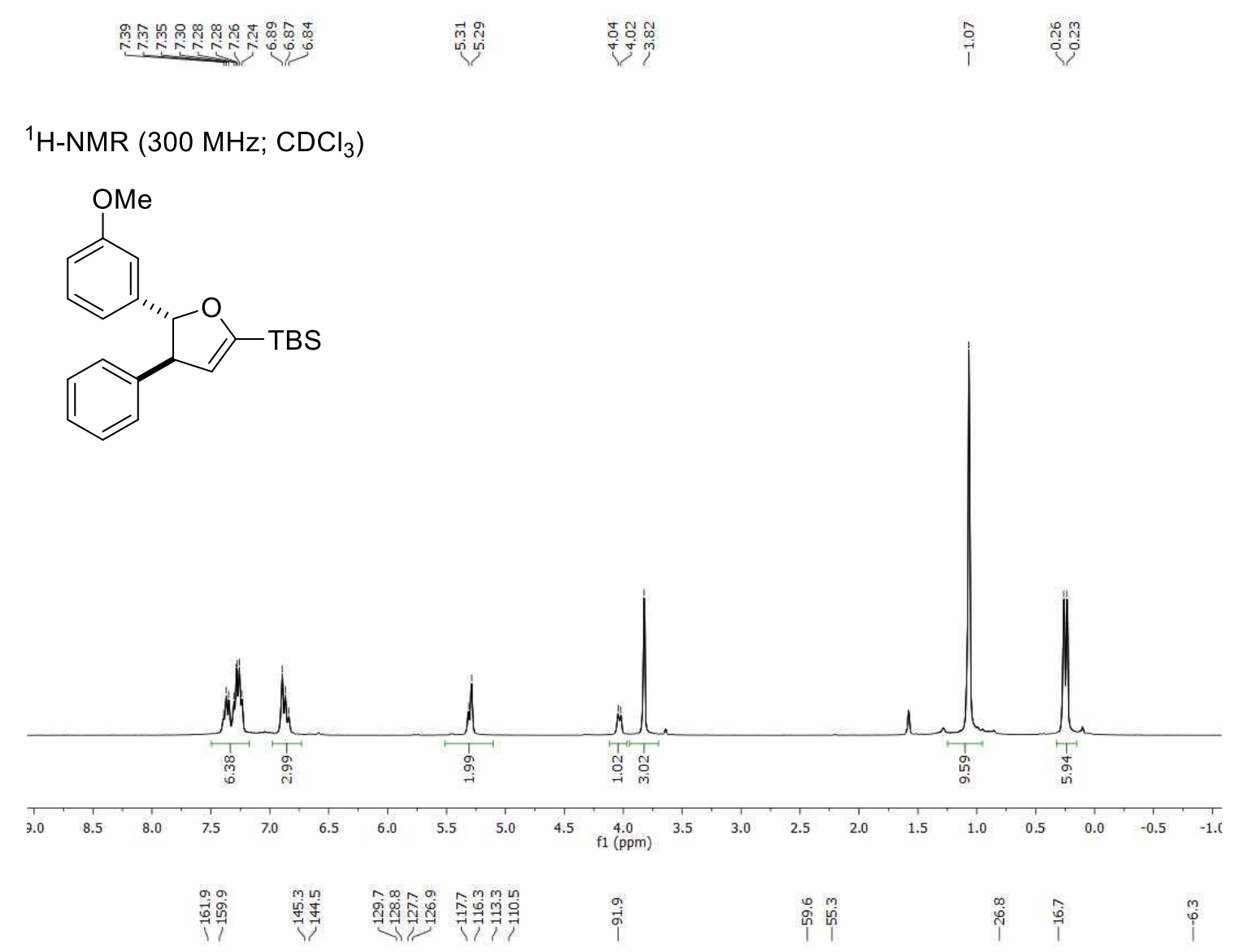

${ }^{13} \mathrm{C}-\mathrm{NMR}\left(75 \mathrm{MHz} ; \mathrm{CDCl}_{3}\right.$ )
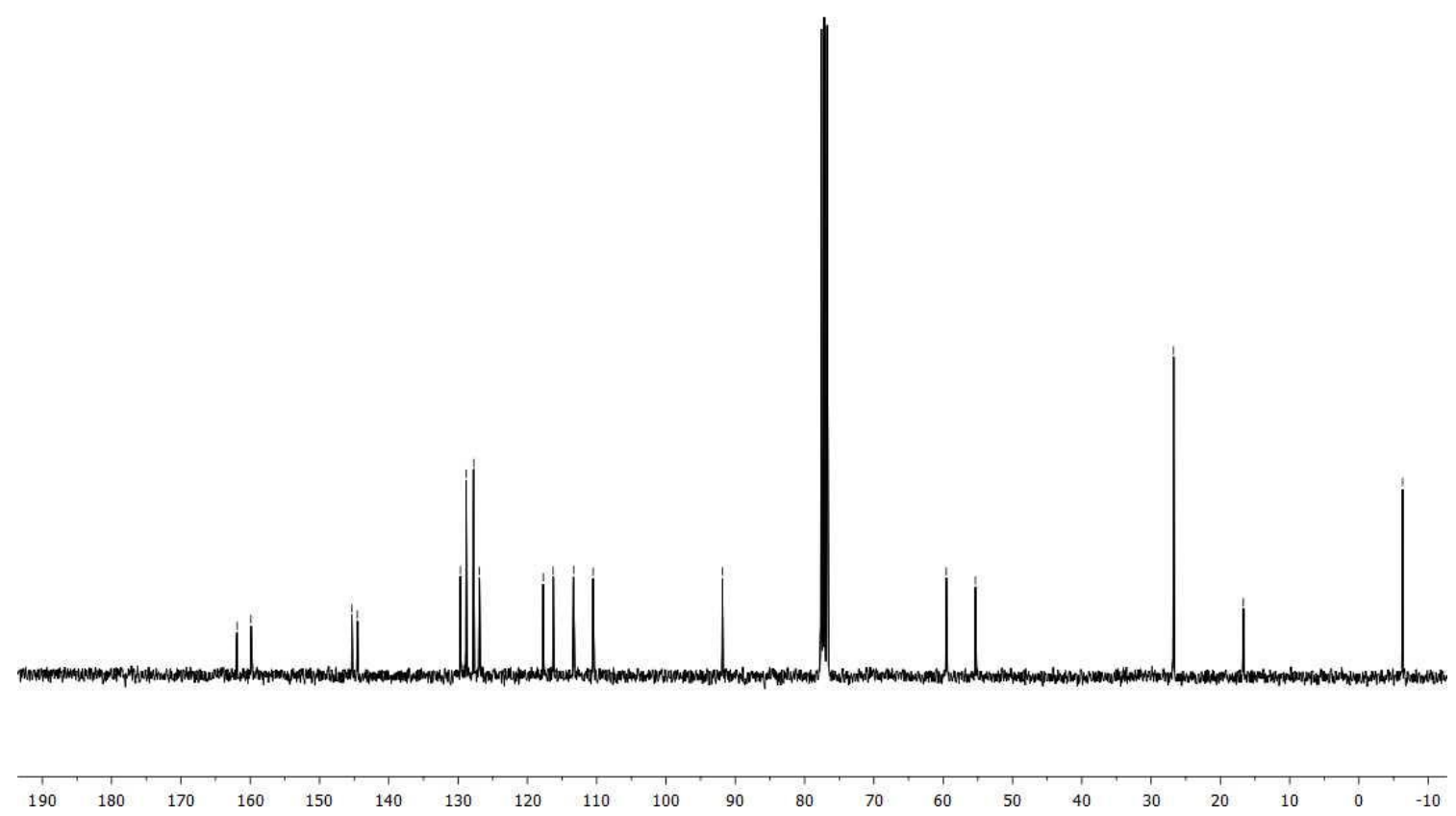
Trans-tert-butyl(5-(3-chlorophenyl)-4-phenyl-4,5-dihydrofuran-2-yl)dimethylsilane (4n).

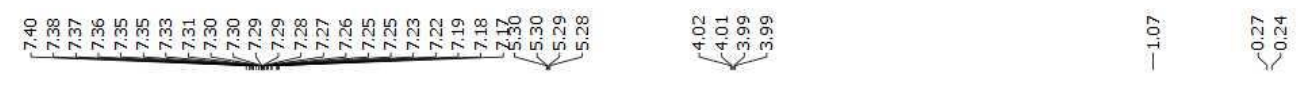

${ }^{1} \mathrm{H}-\mathrm{NMR}\left(300 \mathrm{MHz} ; \mathrm{CDCl}_{3}\right)$

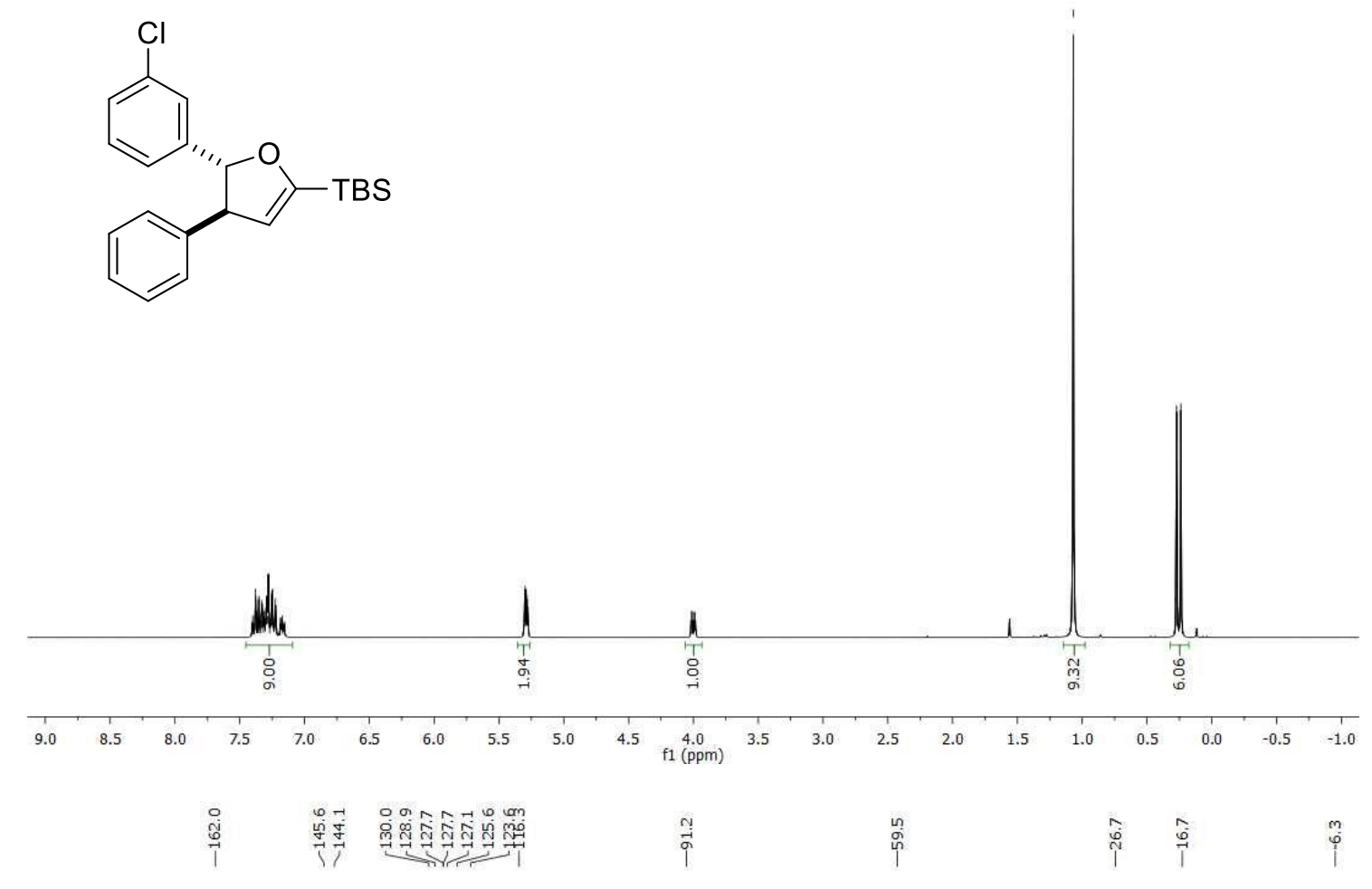

${ }^{13} \mathrm{C}-\mathrm{NMR}\left(75 \mathrm{MHz} ; \mathrm{CDCl}_{3}\right)$

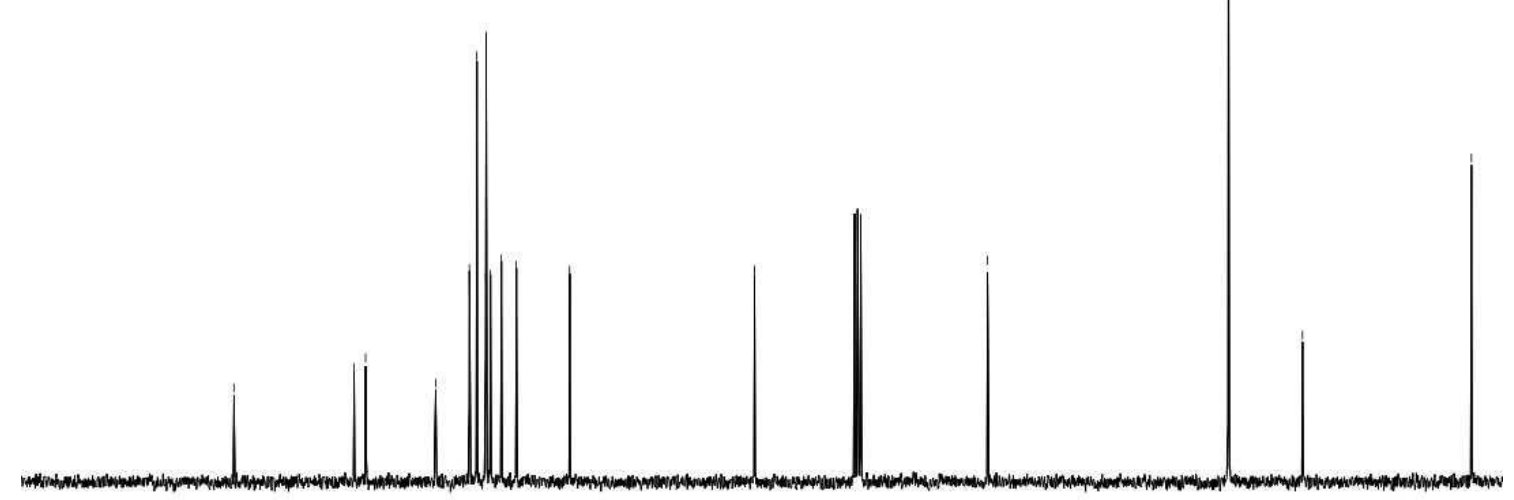

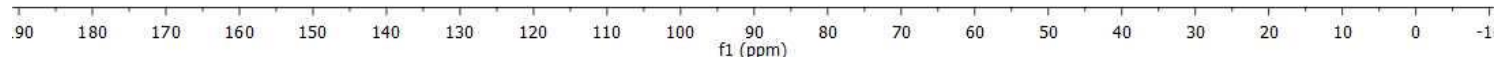


Trans-tert-butyl(5-(3,5-dimethylphenyl)-4-phenyl-4,5-dihydrofuran-2-yl)dimethylsilane (40).

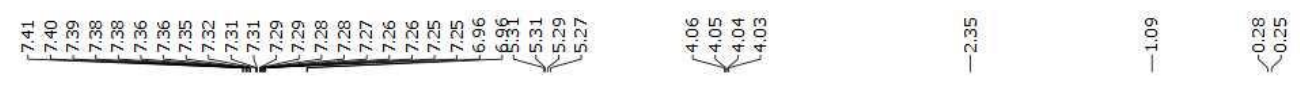

${ }^{1} \mathrm{H}-\mathrm{NMR}\left(300 \mathrm{MHz} ; \mathrm{CDCl}_{3}\right)$
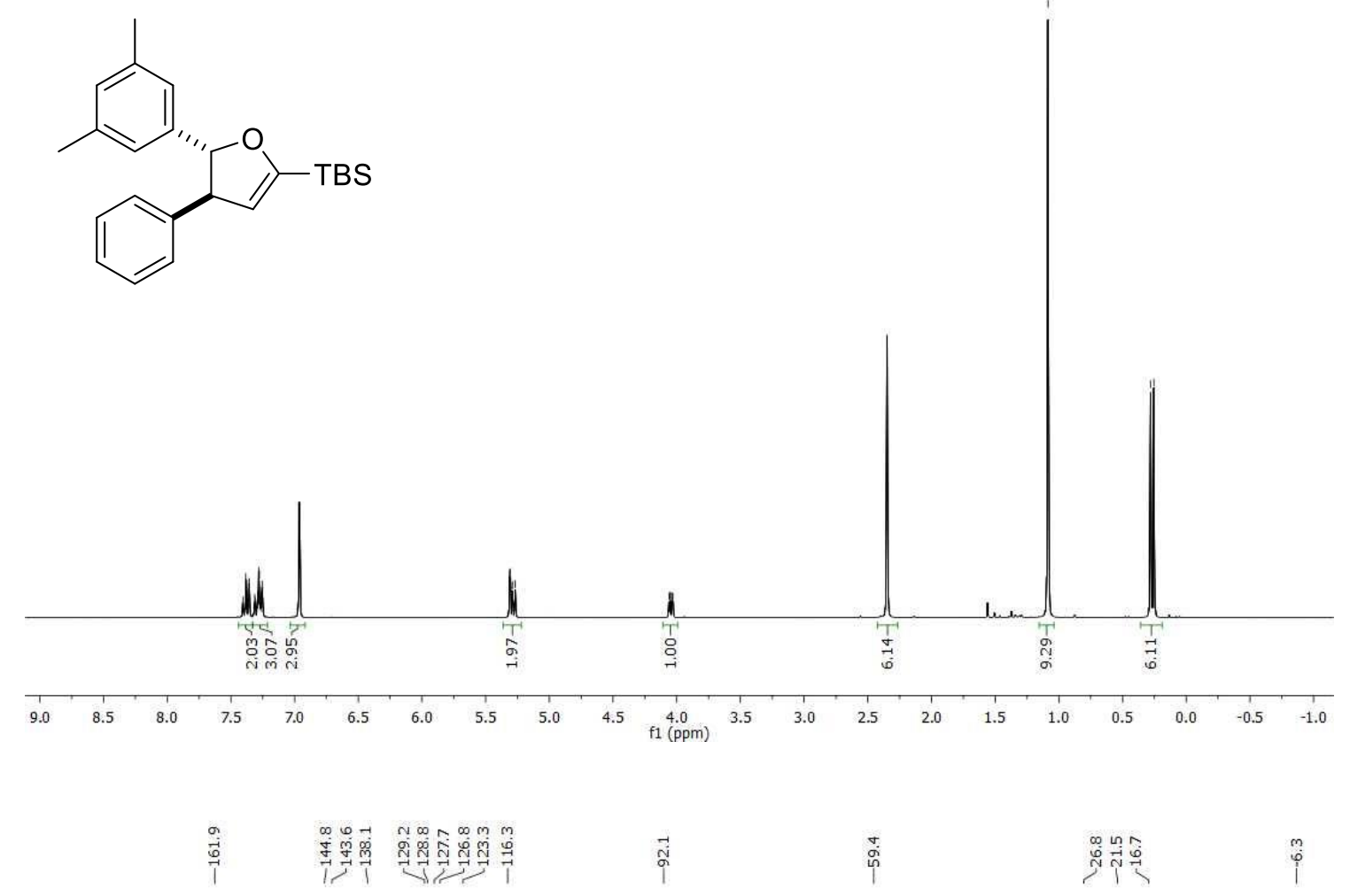

${ }^{13} \mathrm{C}-\mathrm{NMR}\left(75 \mathrm{MHz} ; \mathrm{CDCl}_{3}\right)$

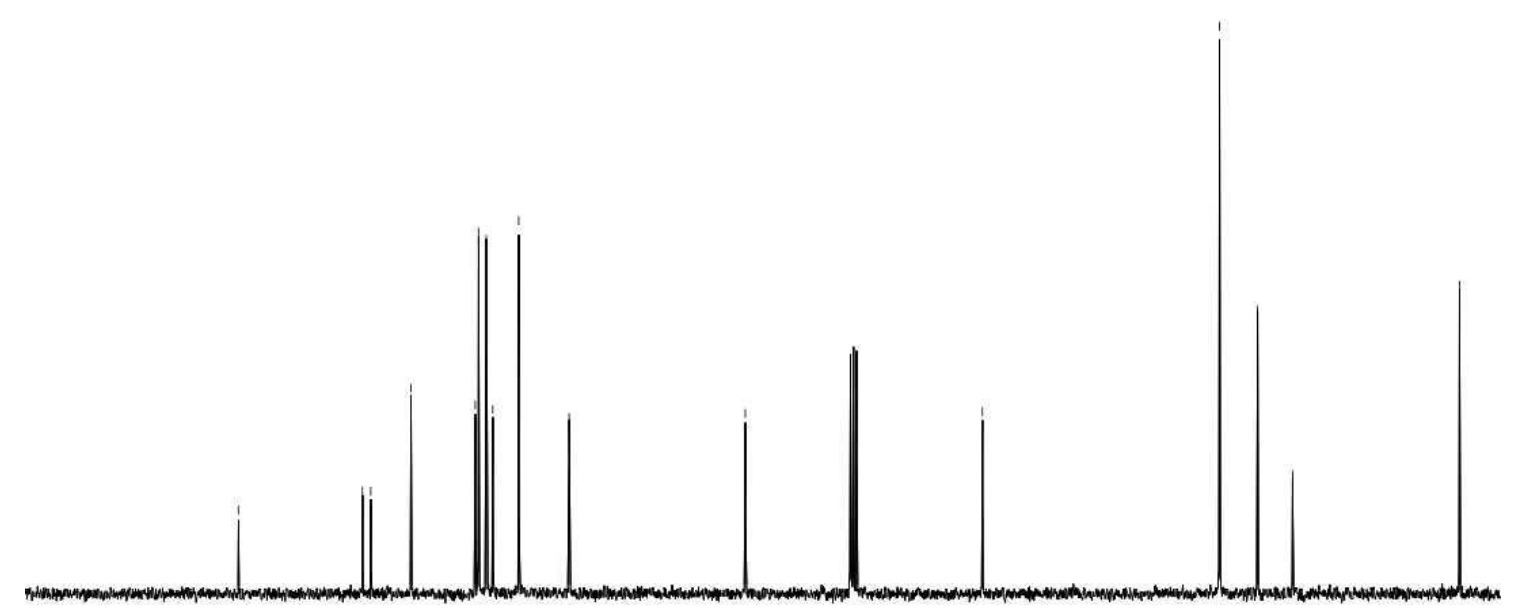

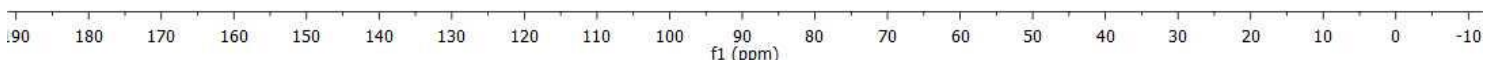


Trans-tert-butyl(5-(3,5-dibromophenyl)-4-phenyl-4,5-dihydrofuran-2-yl)dimethylsilane (4p).

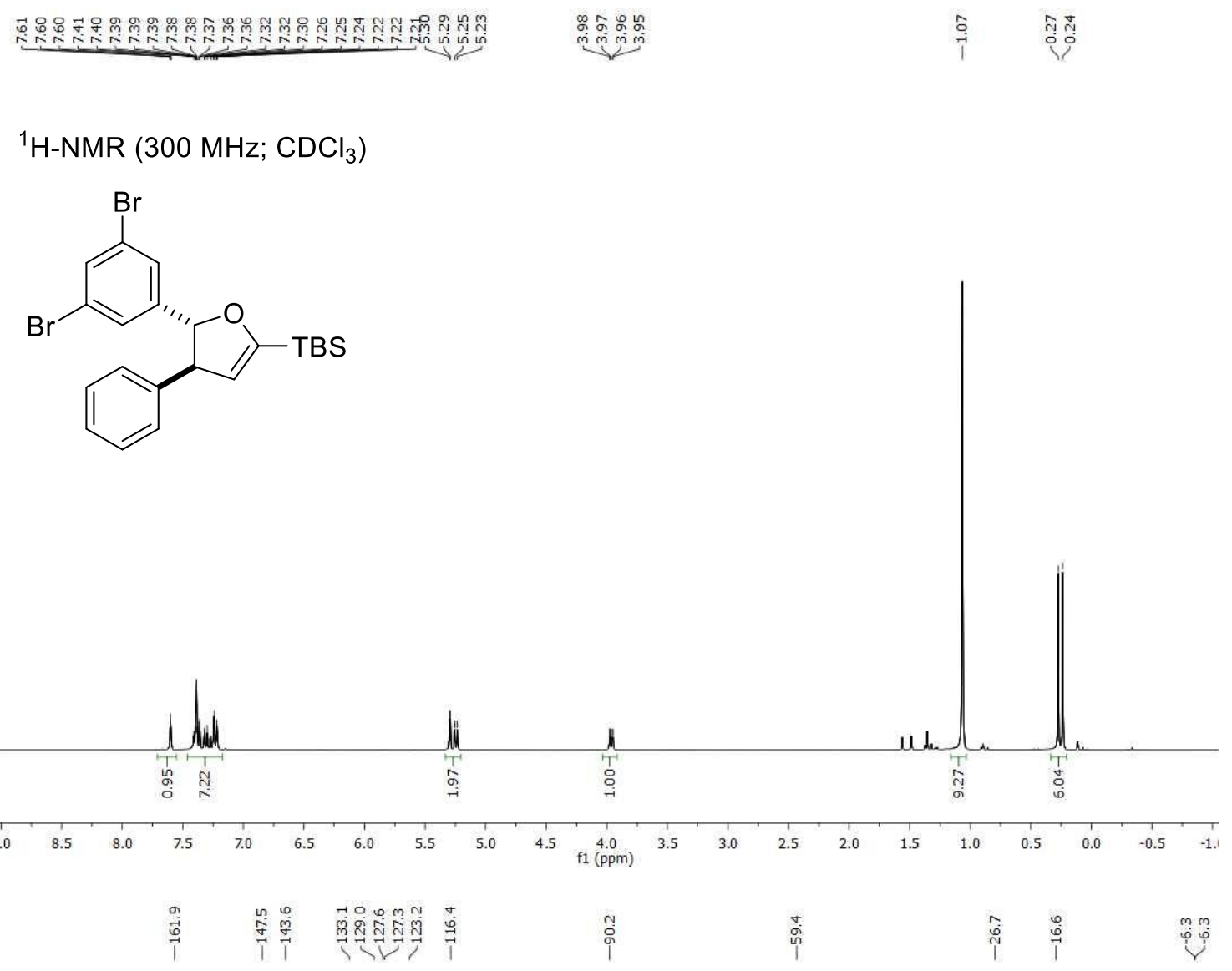

${ }^{13} \mathrm{C}-\mathrm{NMR}\left(75 \mathrm{MHz} ; \mathrm{CDCl}_{3}\right)$
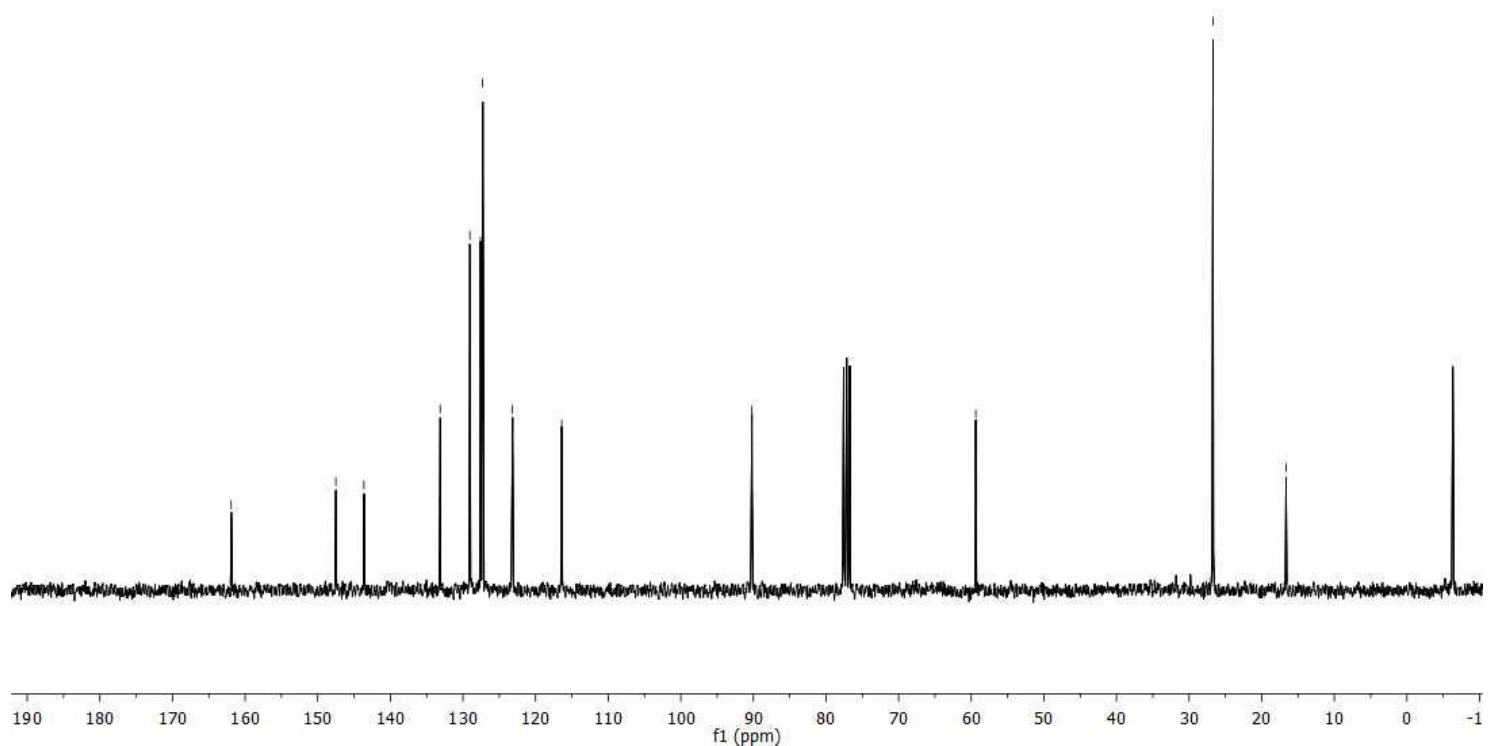
Trans-tert-butyldimethyl(4-phenyl-5-(o-tolyl)-4,5-dihydrofuran-2-yl)silane (4q).

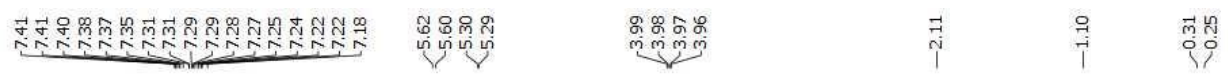

${ }^{1} \mathrm{H}-\mathrm{NMR}\left(300 \mathrm{MHz} ; \mathrm{CDCl}_{3}\right)$

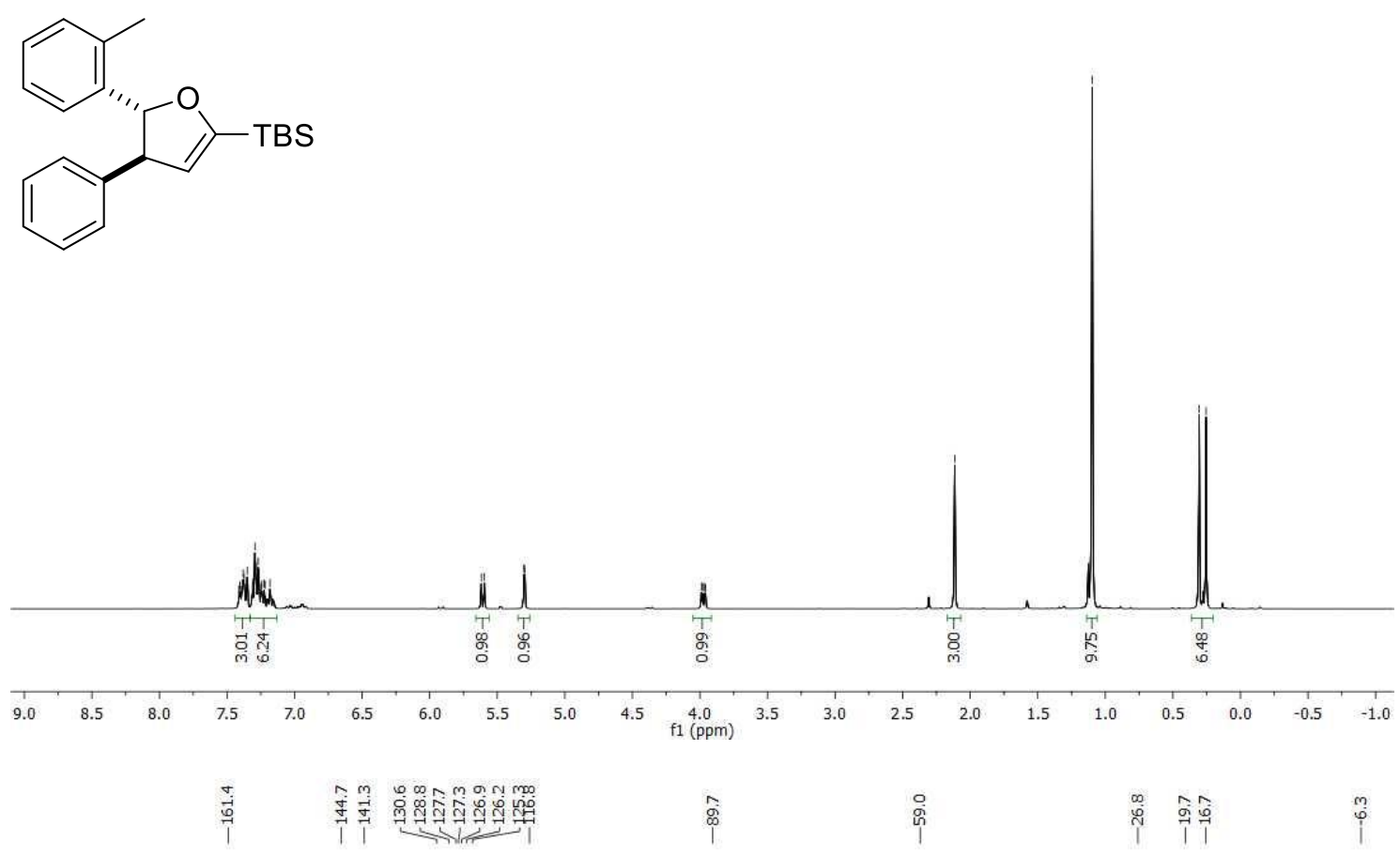

${ }^{13} \mathrm{C}-\mathrm{NMR}\left(75 \mathrm{MHz} ; \mathrm{CDCl}_{3}\right)$

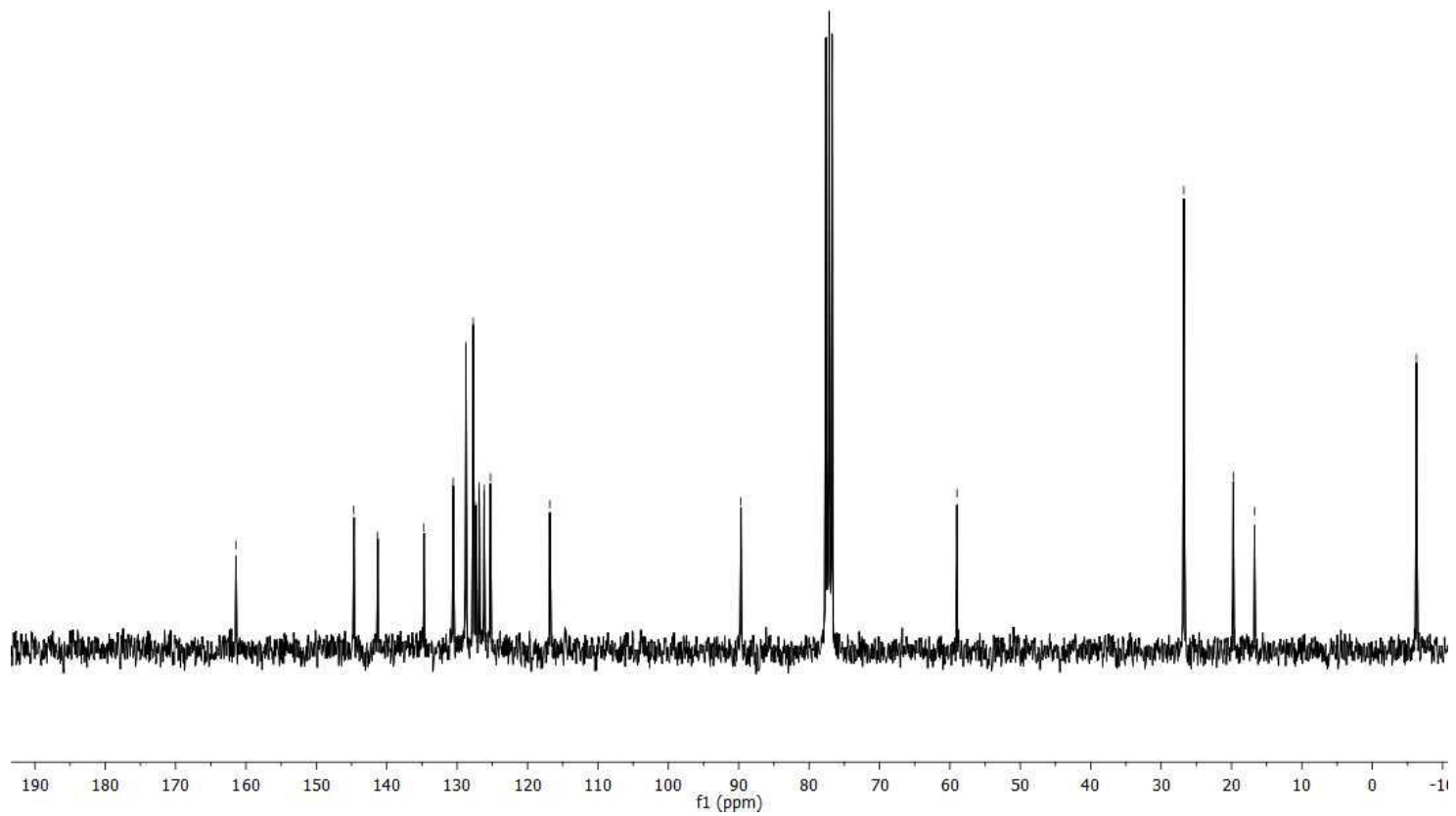


Trans-tert-butyldimethyl(5-(naphthalen-1-yl)-4-phenyl-4,5-dihydrofuran-2-yl)silane (4r).

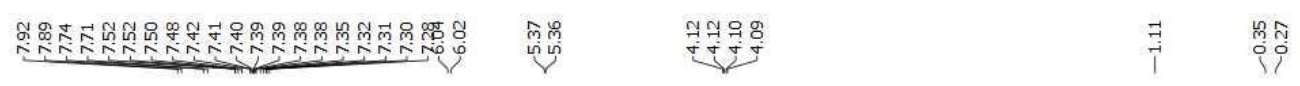

${ }^{1} \mathrm{H}-\mathrm{NMR}\left(300 \mathrm{MHz} ; \mathrm{CDCl}_{3}\right)$

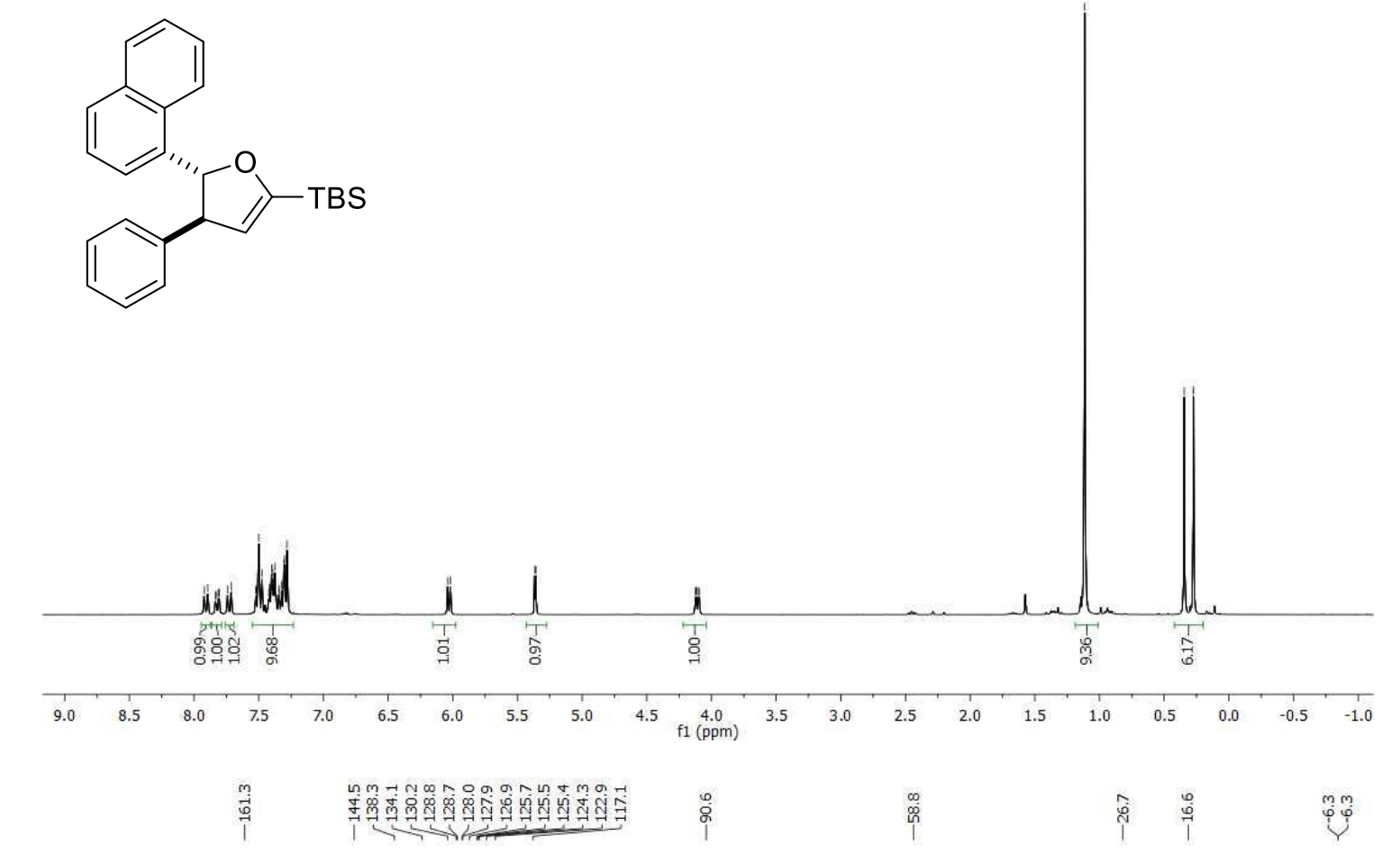

${ }^{13} \mathrm{C}-\mathrm{NMR}\left(75 \mathrm{MHz} ; \mathrm{CDCl}_{3}\right)$

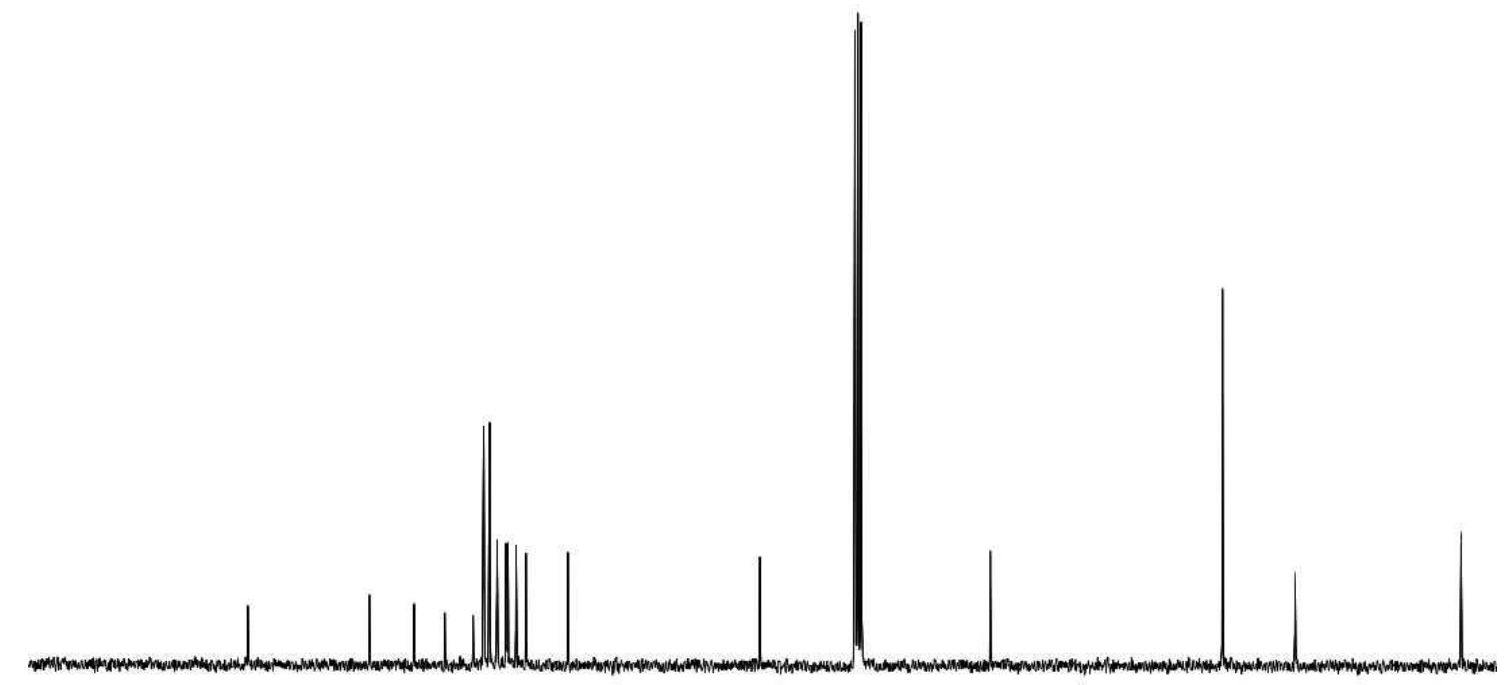

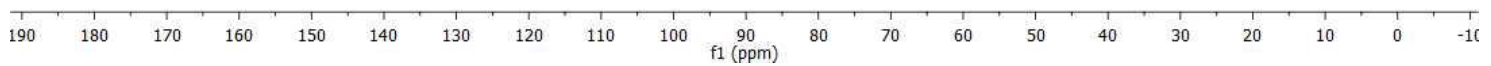


Trans-tert-butyl(-4,5-di-p-tolyl-4,5-dihydrofuran-2-yl)dimethylsilane (4s).

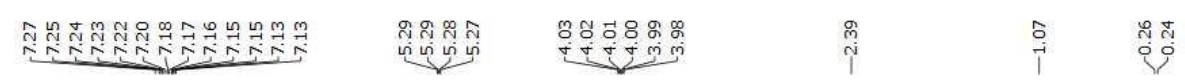

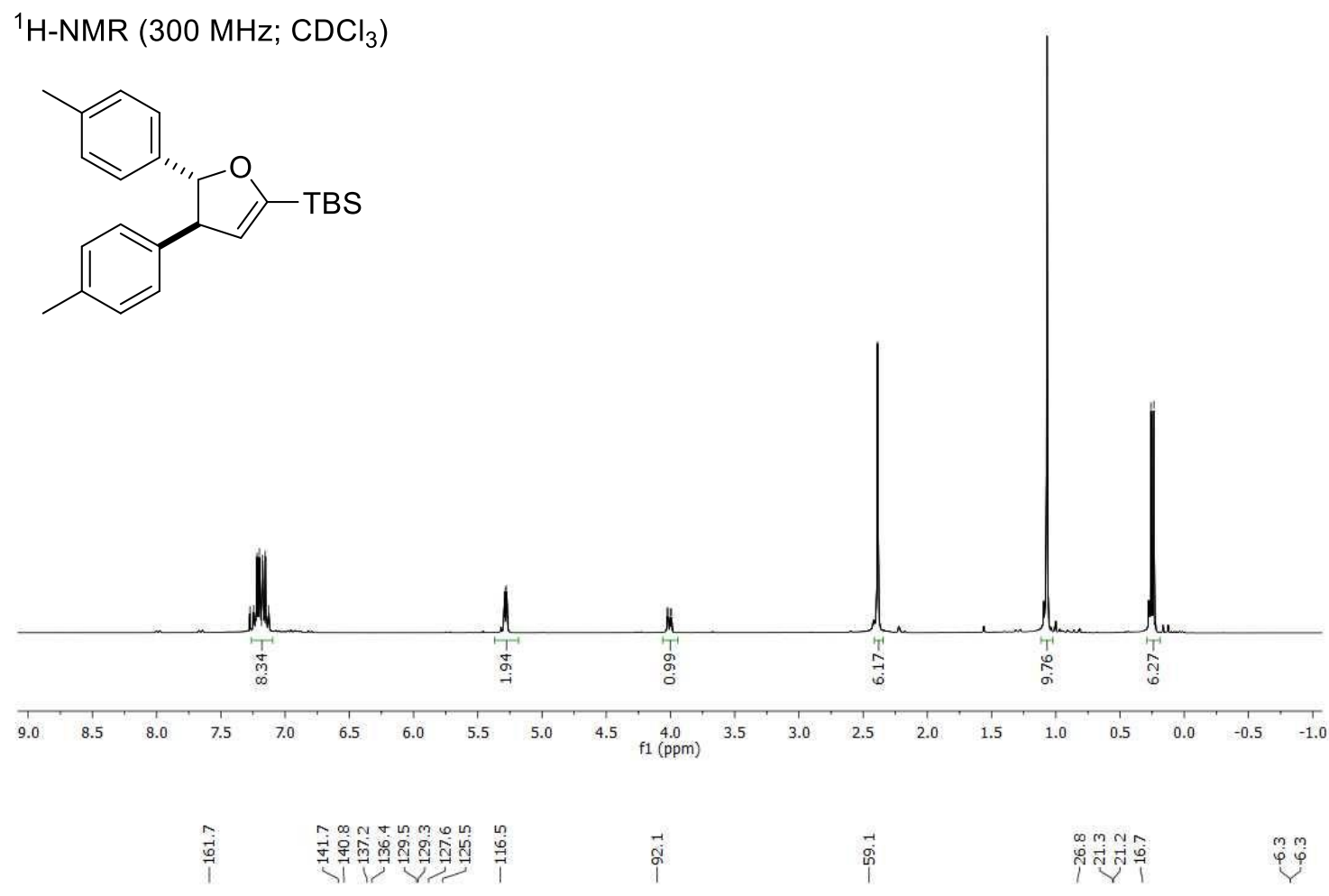

${ }^{13} \mathrm{C}-\mathrm{NMR}\left(75 \mathrm{MHz} ; \mathrm{CDCl}_{3}\right)$

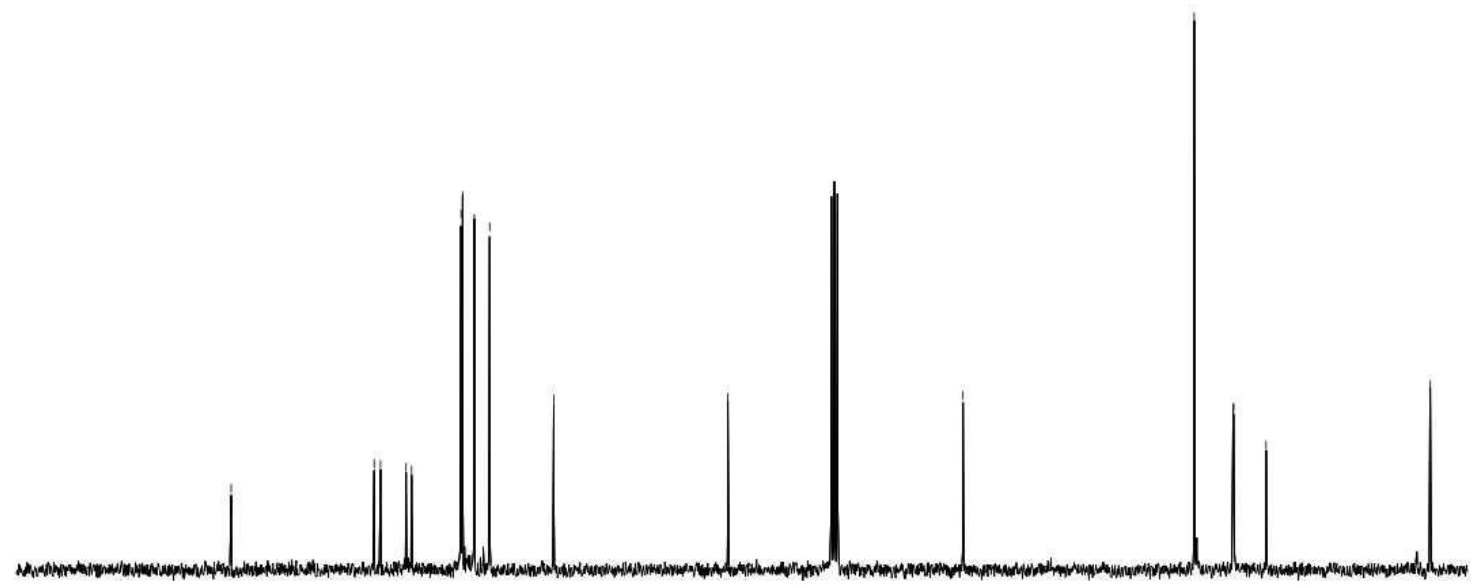

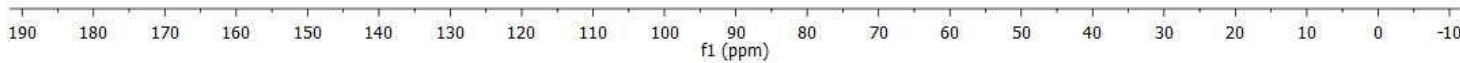


Trans-triisopropyl(4-phenyl-5-(p-tolyl)-4,5-dihydrofuran-2-yl)silane (4t).
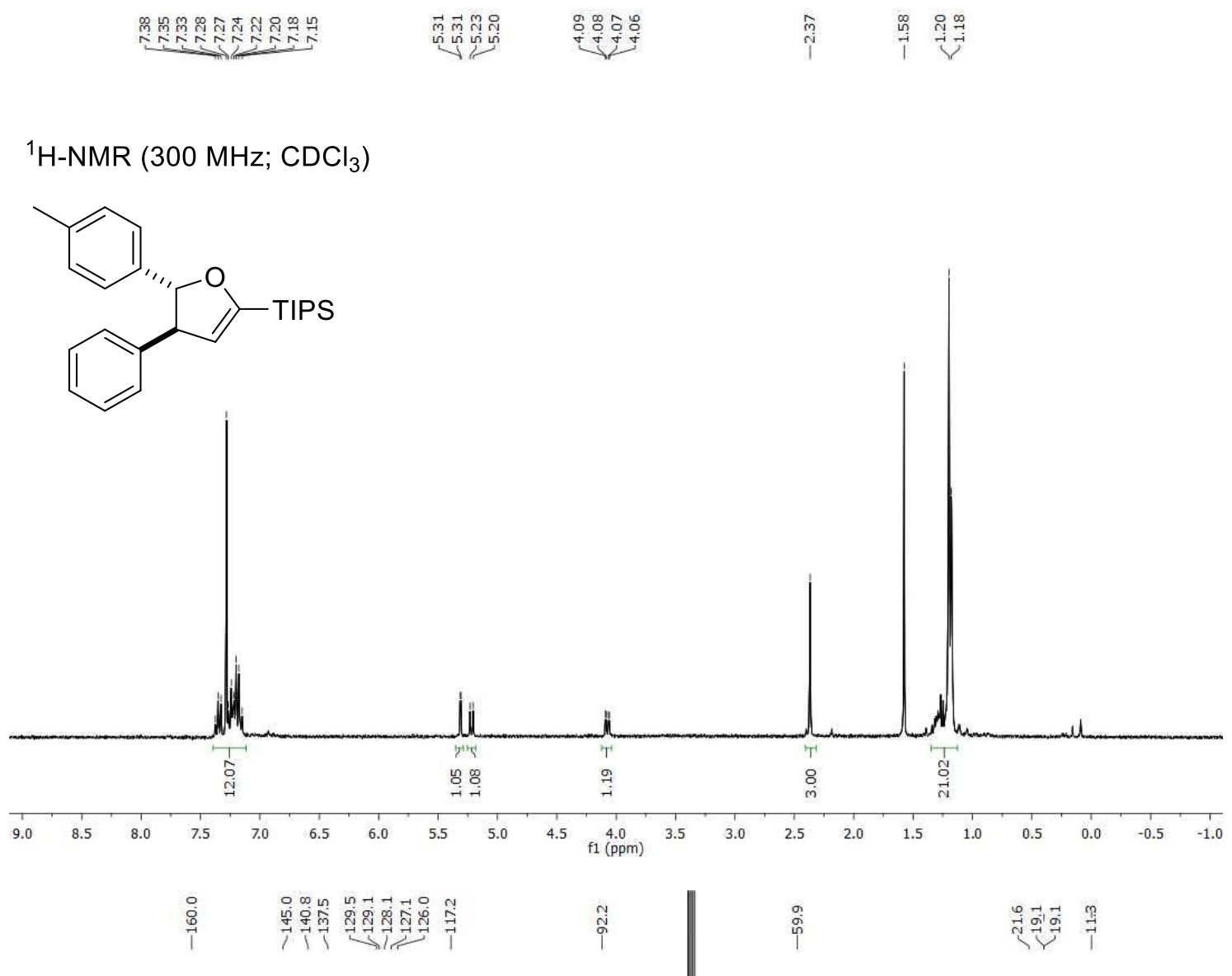

${ }^{13} \mathrm{C}-\mathrm{NMR}\left(75 \mathrm{MHz} ; \mathrm{CDCl}_{3}\right)$

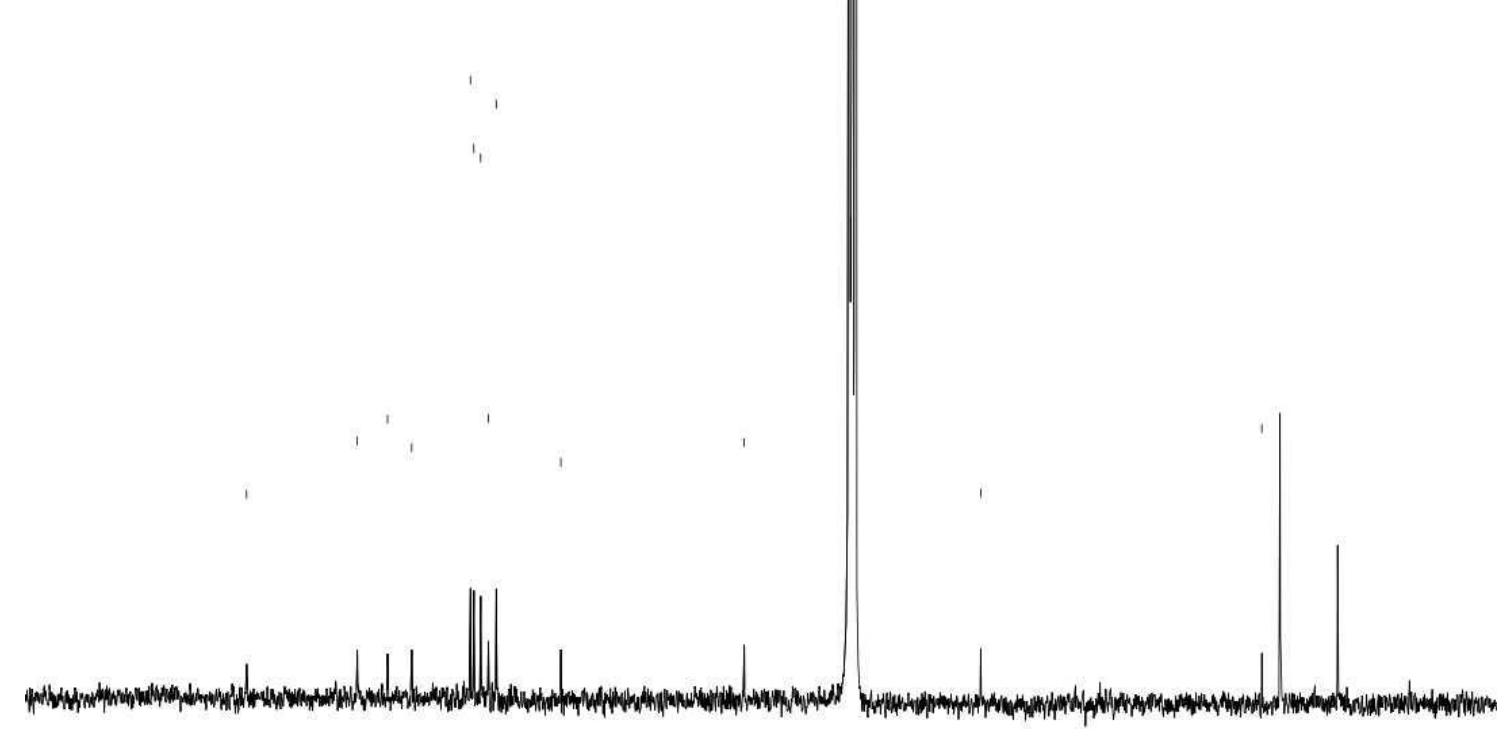

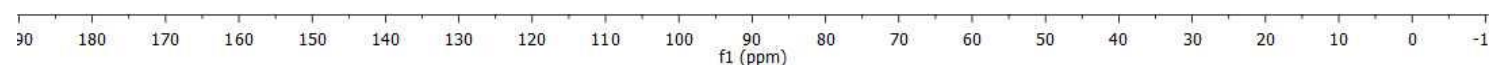


Trans-tert-butyl(4-butyl-5-( $p$-tolyl)-4,5-dihydrofuran-2-yl)dimethylsilane (4u).

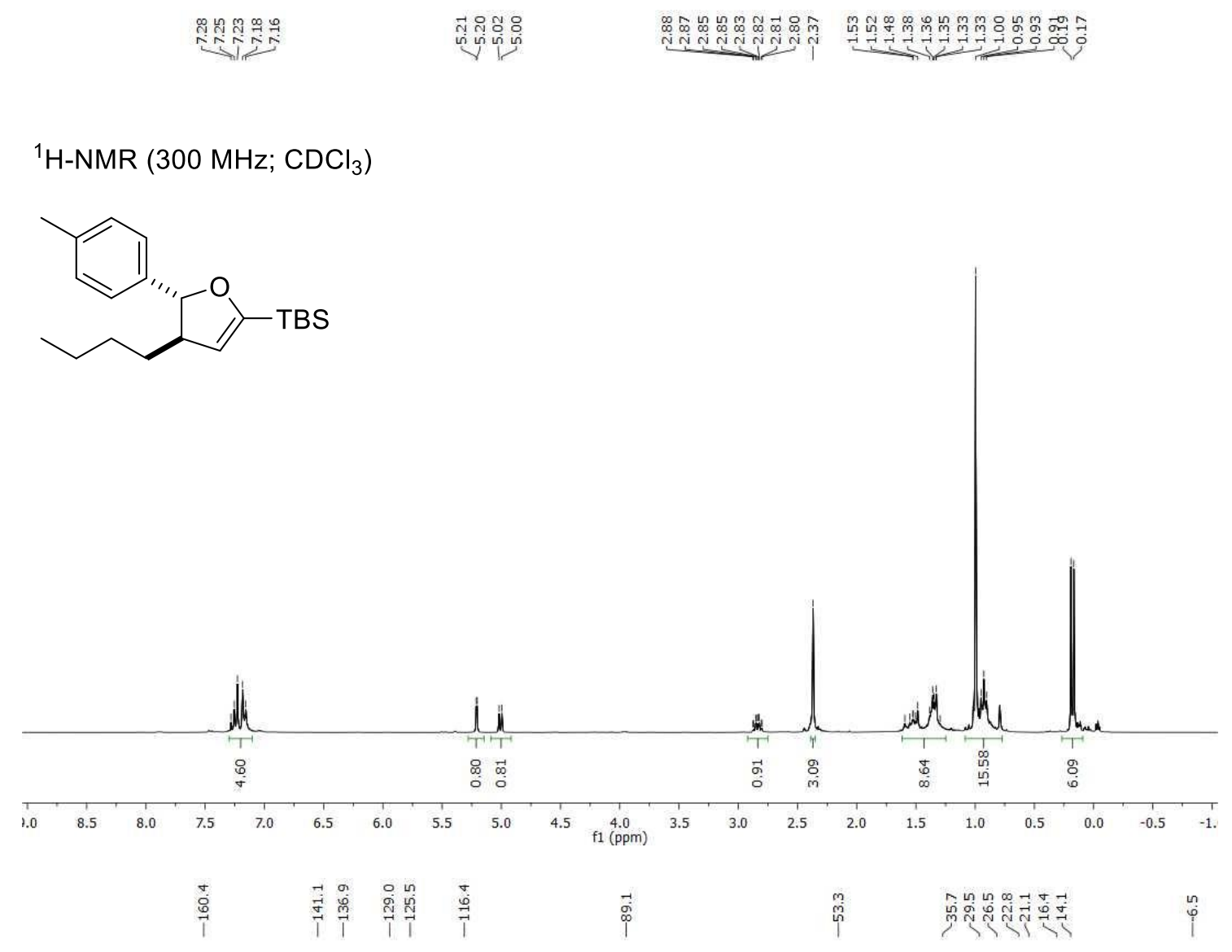

${ }^{13} \mathrm{C}-\mathrm{NMR}\left(75 \mathrm{MHz} ; \mathrm{CDCl}_{3}\right)$

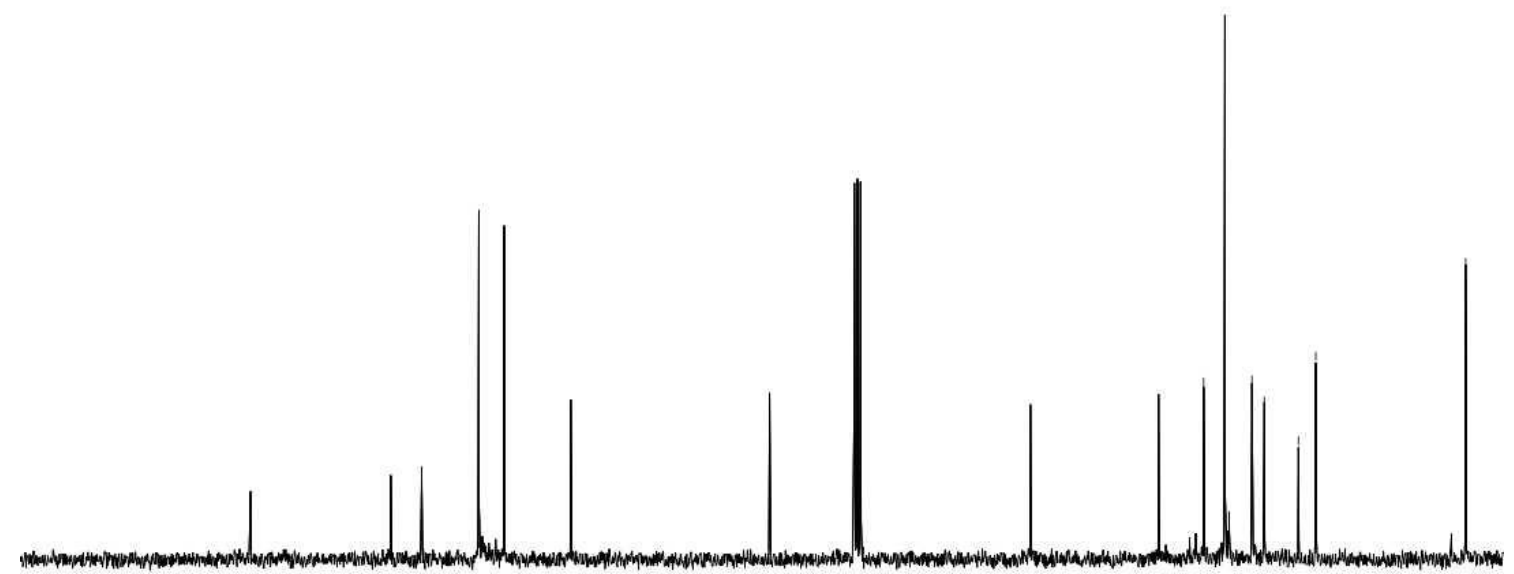

$\begin{array}{llllllllllllllllllllllllllllll}190 & 180 & 170 & 160 & 150 & 140 & 130 & 120 & 110 & 100 & 90 & 80 & 70 & 60 & 50 & 40 & 30 & 20 & 10 & 0 & -10\end{array}$ 
Trans-tert-butyl(4-cyclopentyl-5-(p-tolyl)-4,5-dihydrofuran-2-yl)dimethylsilane (4v).

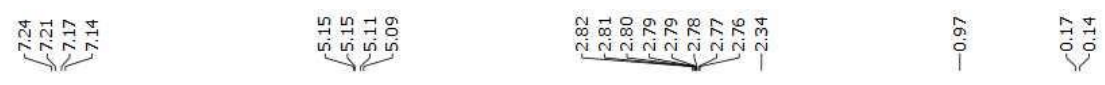

${ }^{1} \mathrm{H}-\mathrm{NMR}\left(300 \mathrm{MHz} ; \mathrm{CDCl}_{3}\right)$
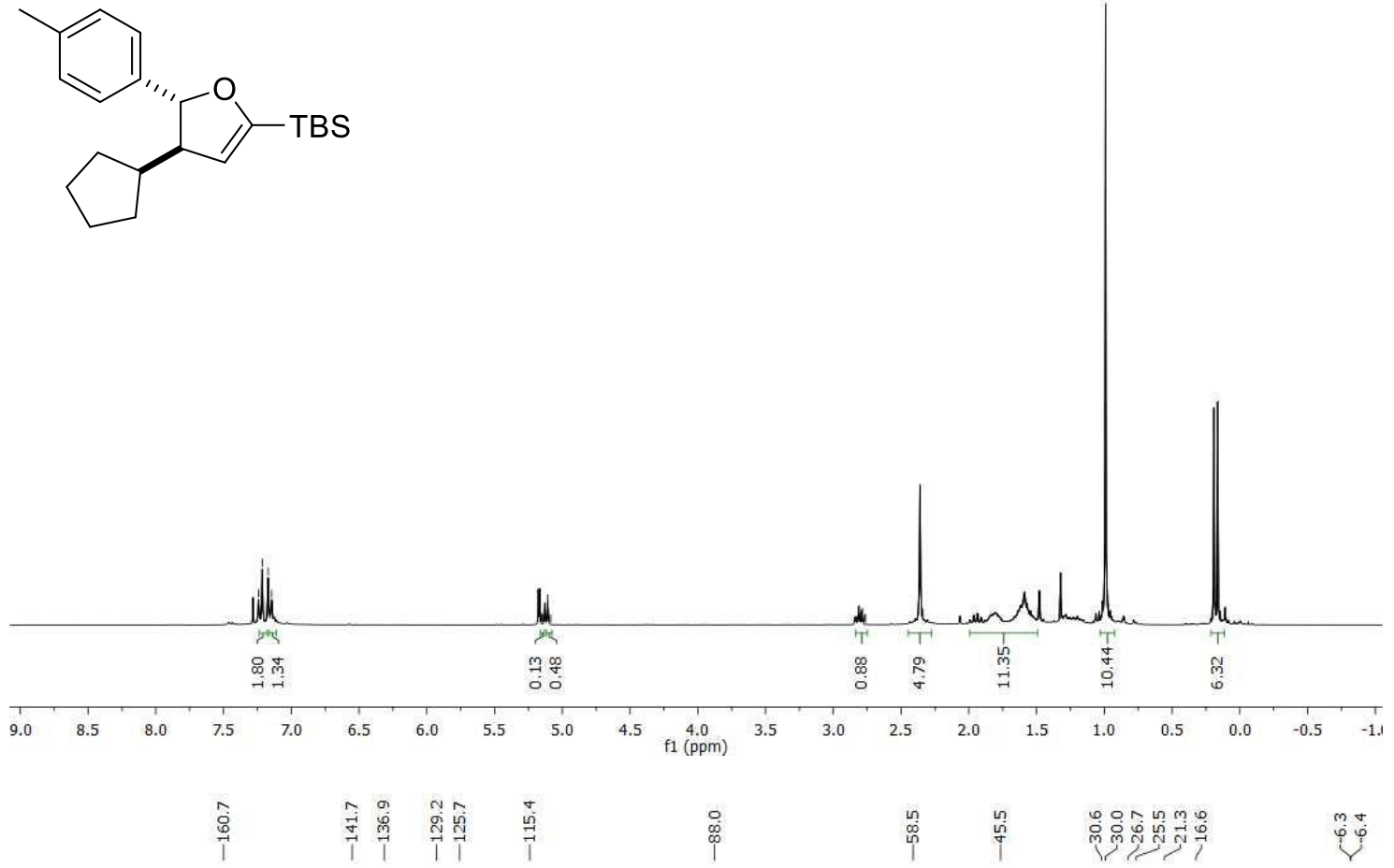

${ }^{13} \mathrm{C}-\mathrm{NMR}\left(75 \mathrm{MHz} ; \mathrm{CDCl}_{3}\right)$

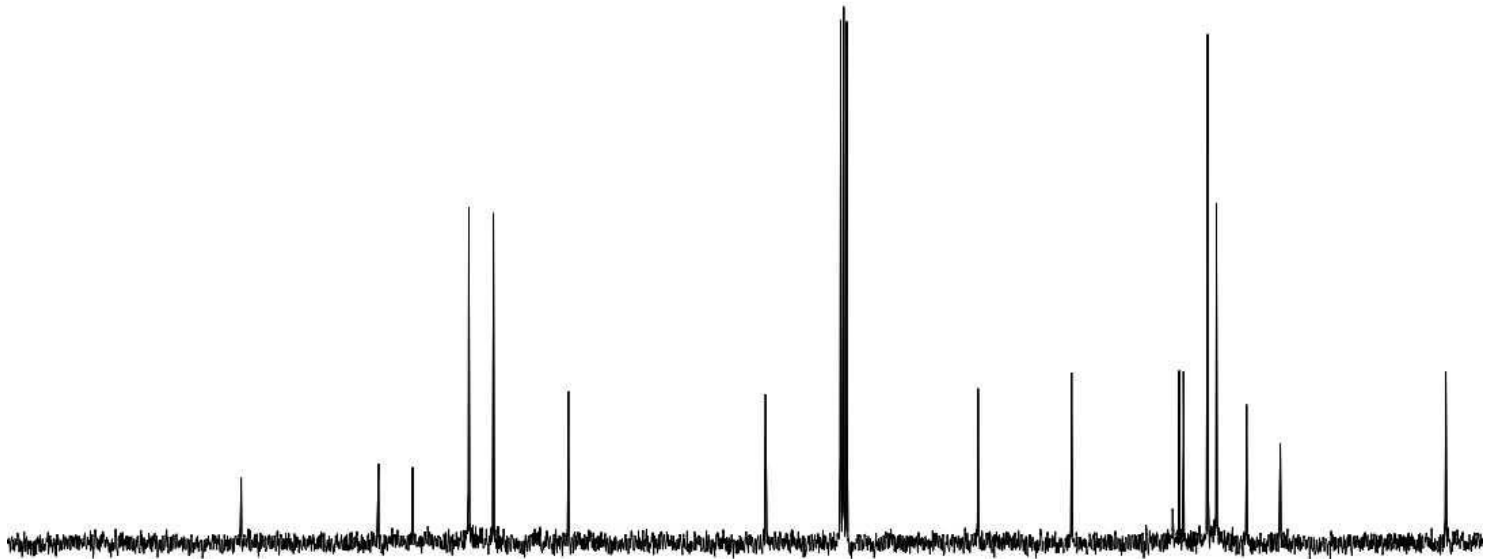

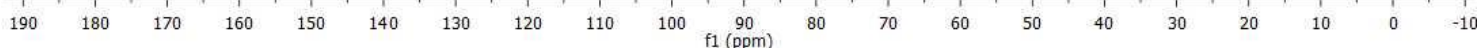


(E)-tert-butyldimethyl(3-deutero-4-phenyl-5-(p-tolyl)-4,5-dihydrofuran-2-yl)silane (3D4b)
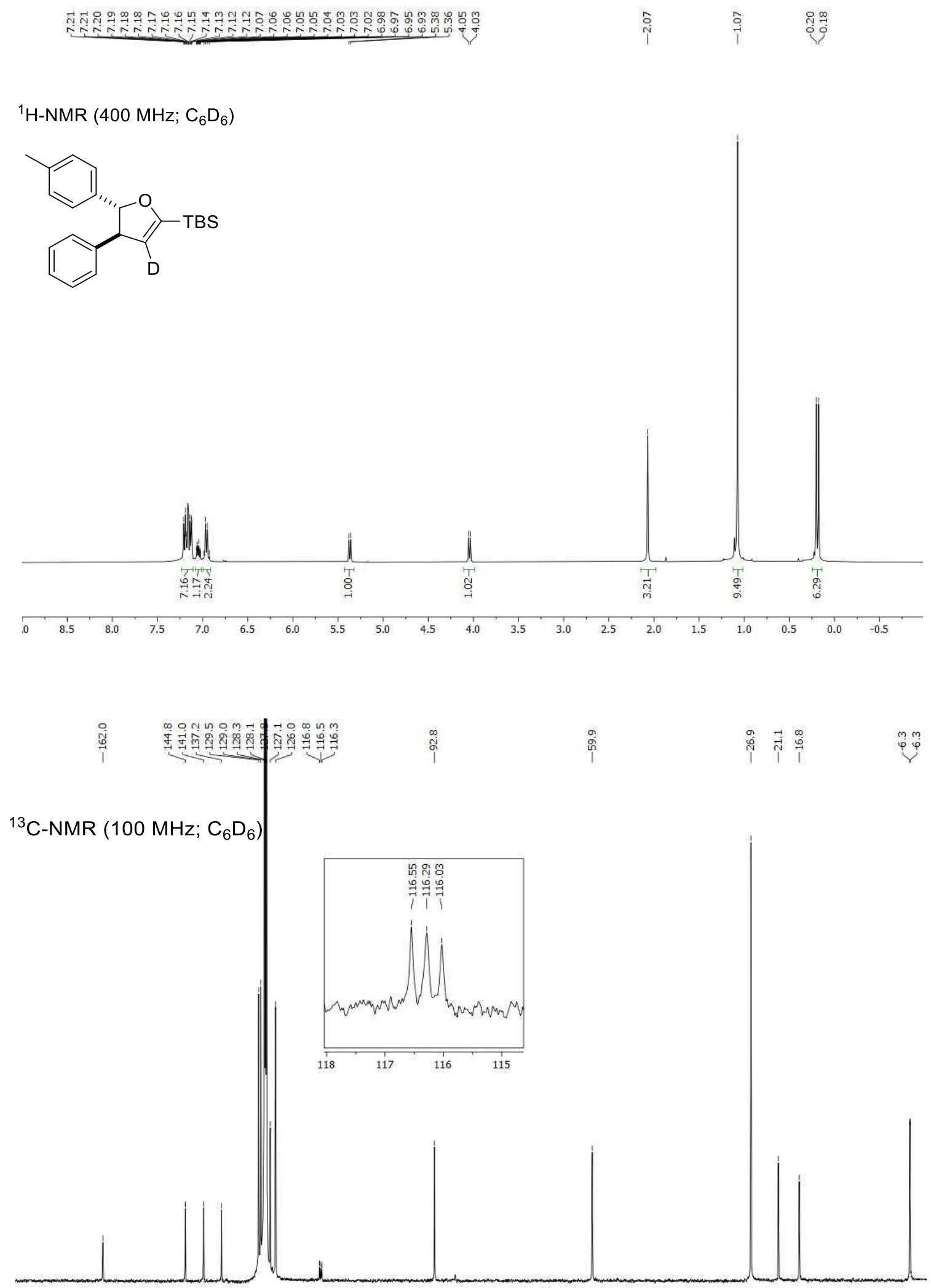

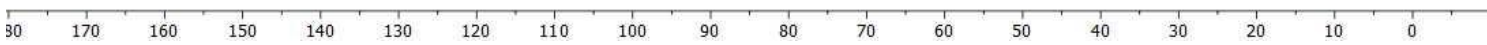


(E)-tert-butyldimethyl(4-deutero-4-phenyl-5-(p-tolyl)-4,5-dihydrofuran-2-yl)silane (4D4b)
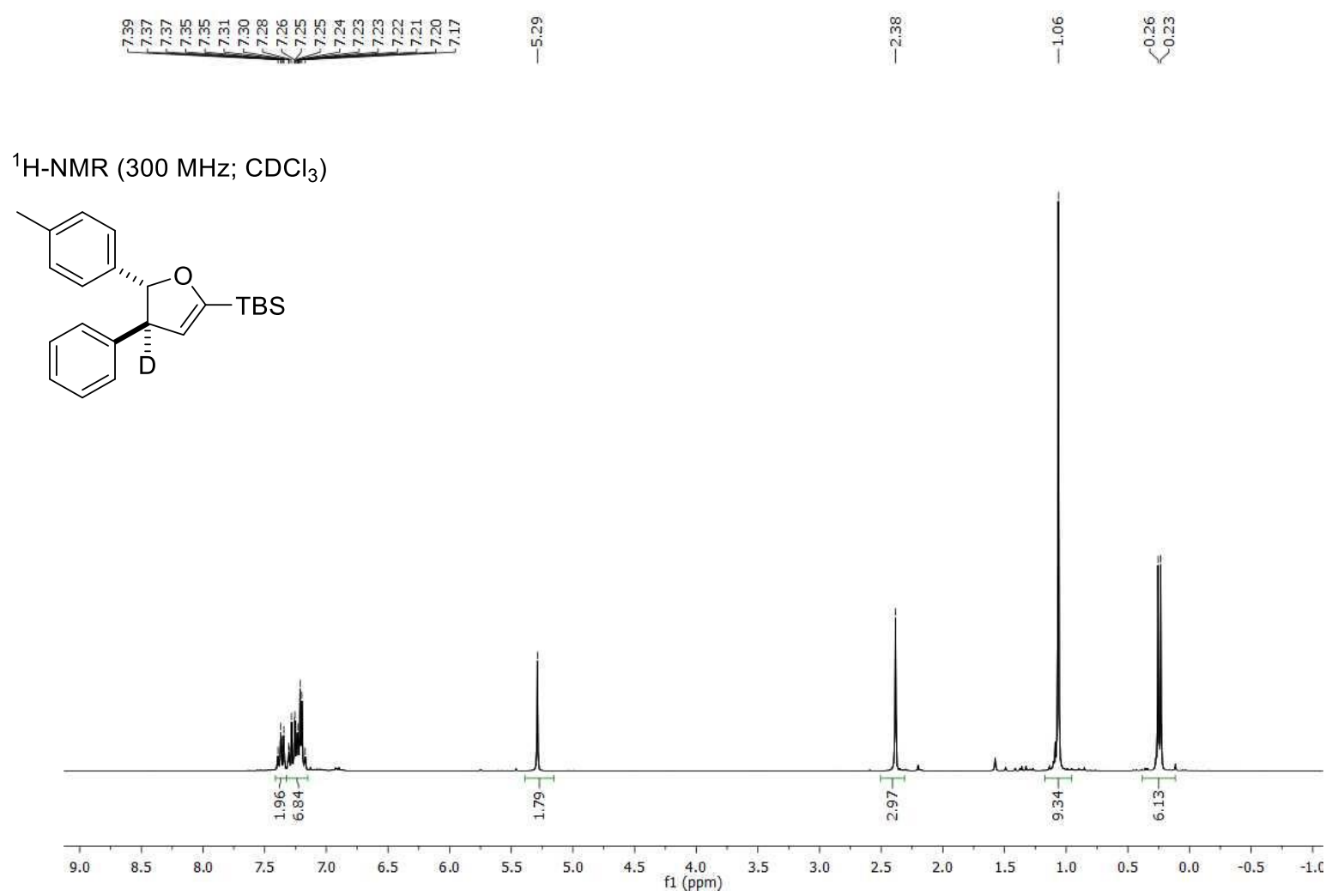

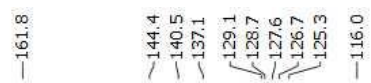
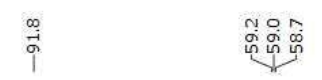

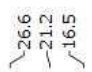

${ }^{13} \mathrm{C}-\mathrm{NMR}\left(75 \mathrm{MHz} ; \mathrm{CDCl}_{3}\right)$

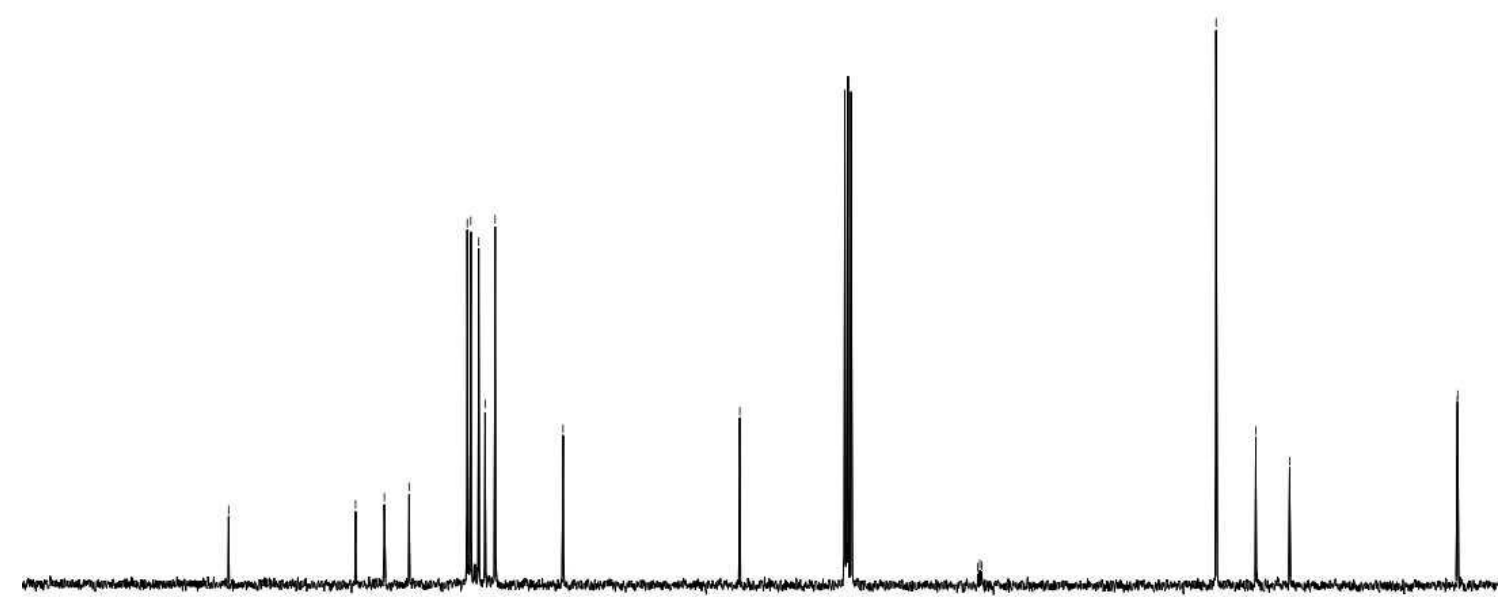

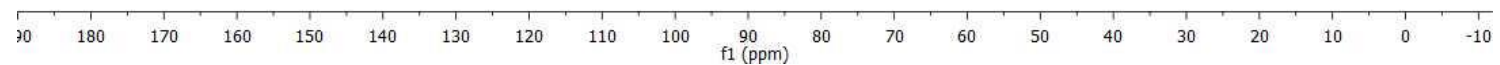


(E)-tert-butyldimethyl(5-deutero-4,5-diphenyl-4,5-dihydrofuran-2-yl)silane (5D-4a)
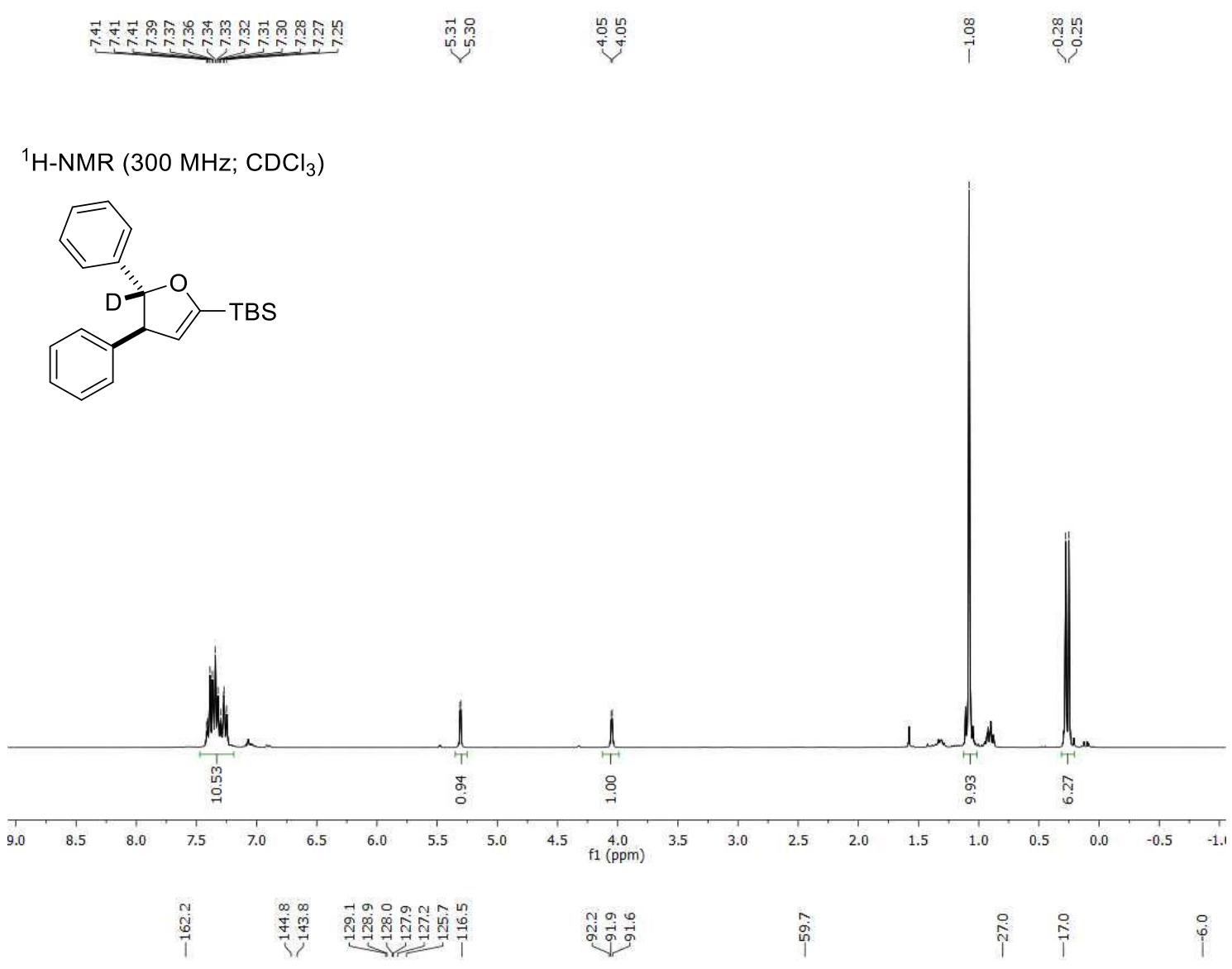

${ }^{13} \mathrm{C}-\mathrm{NMR}\left(75 \mathrm{MHz} ; \mathrm{CDCl}_{3}\right)$

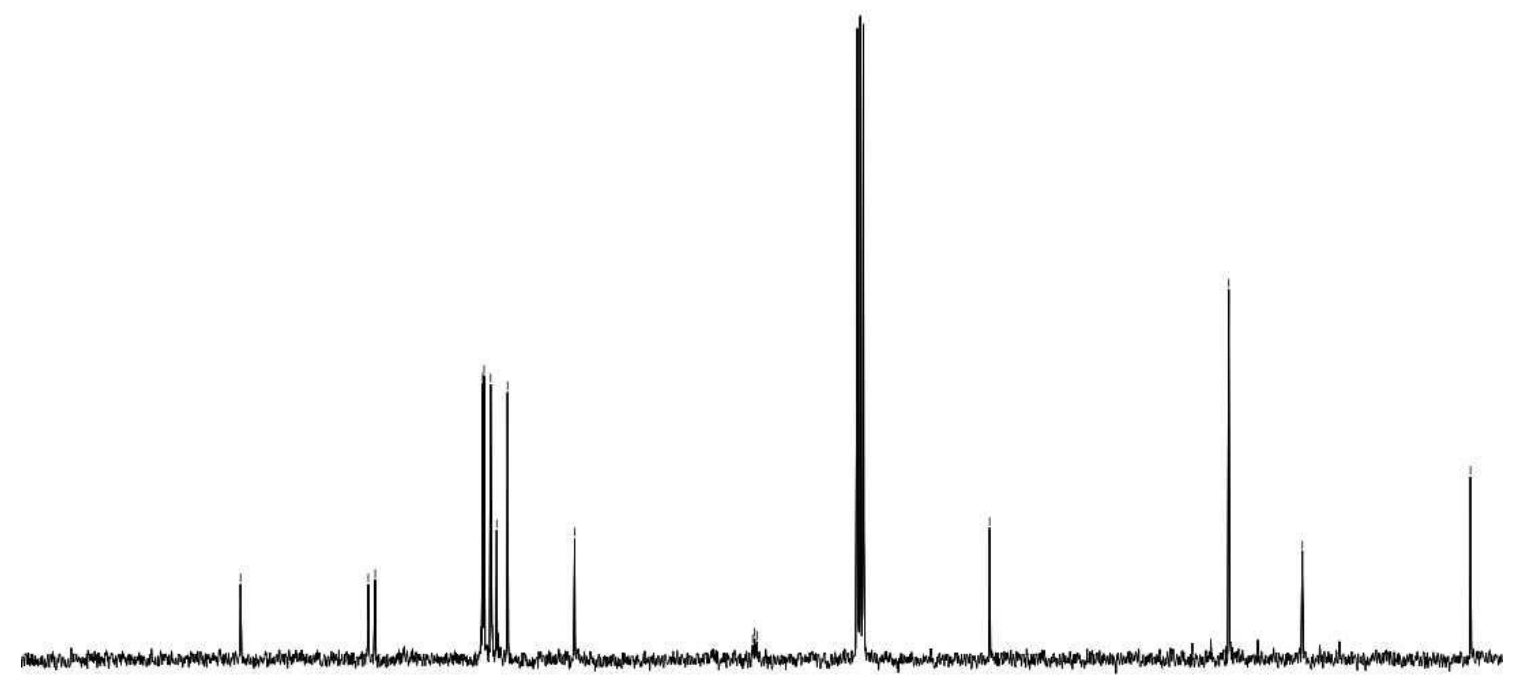

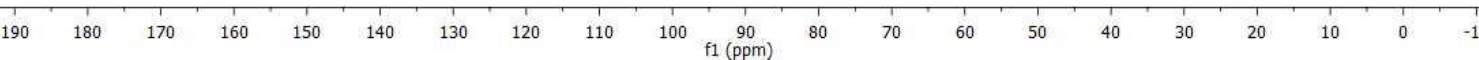




\section{1,3-dimethoxy-6-phenylnaphthalene (6)}

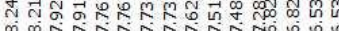

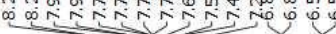

${ }^{1} \mathrm{H}-\mathrm{NMR}\left(300 \mathrm{MHz} ; \mathrm{CDCl}_{3}\right)$<smiles>COc1cc(OC)c2ccc(-c3ccccc3)cc2c1</smiles>

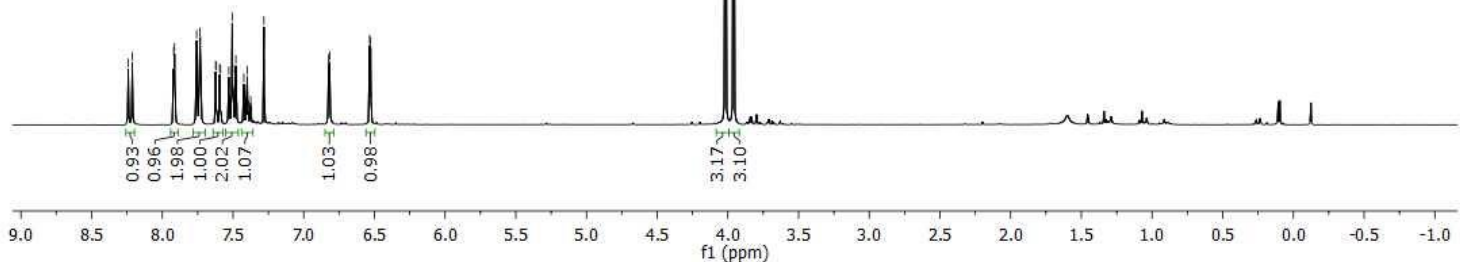

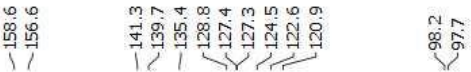

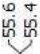

${ }^{13} \mathrm{C}-\mathrm{NMR}\left(75 \mathrm{MHz} ; \mathrm{CDCl}_{3}\right.$ )

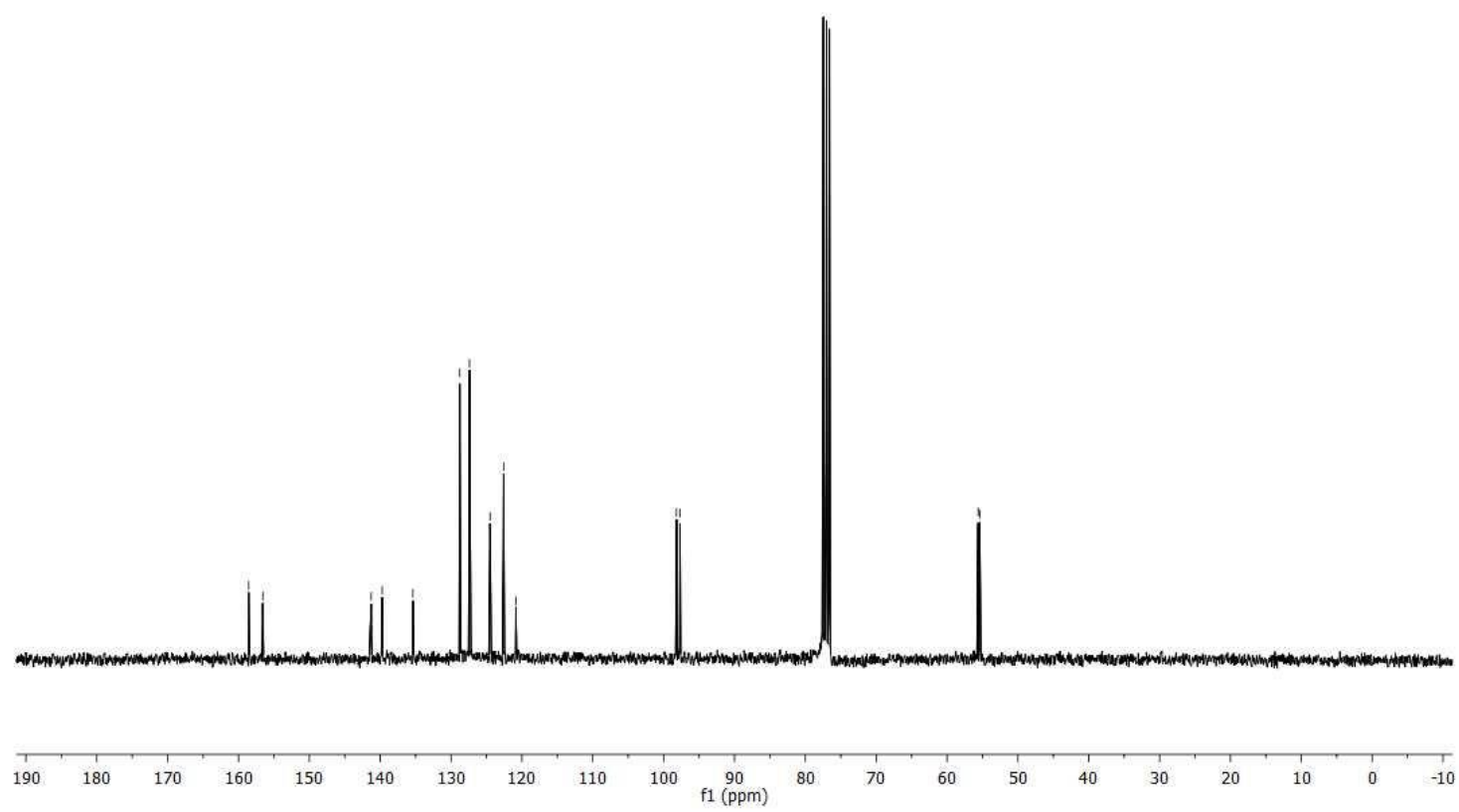


Syn-4-(tert-butyldimethylsilyl)-1-(4-trifluoromethylphenyl)-2-phenylbut-3-yn-1-ol (anti5j)

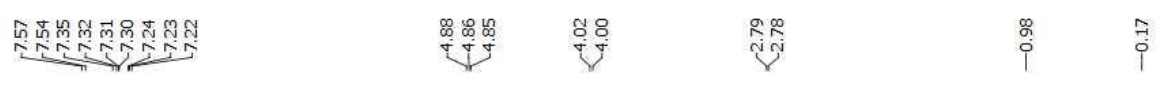
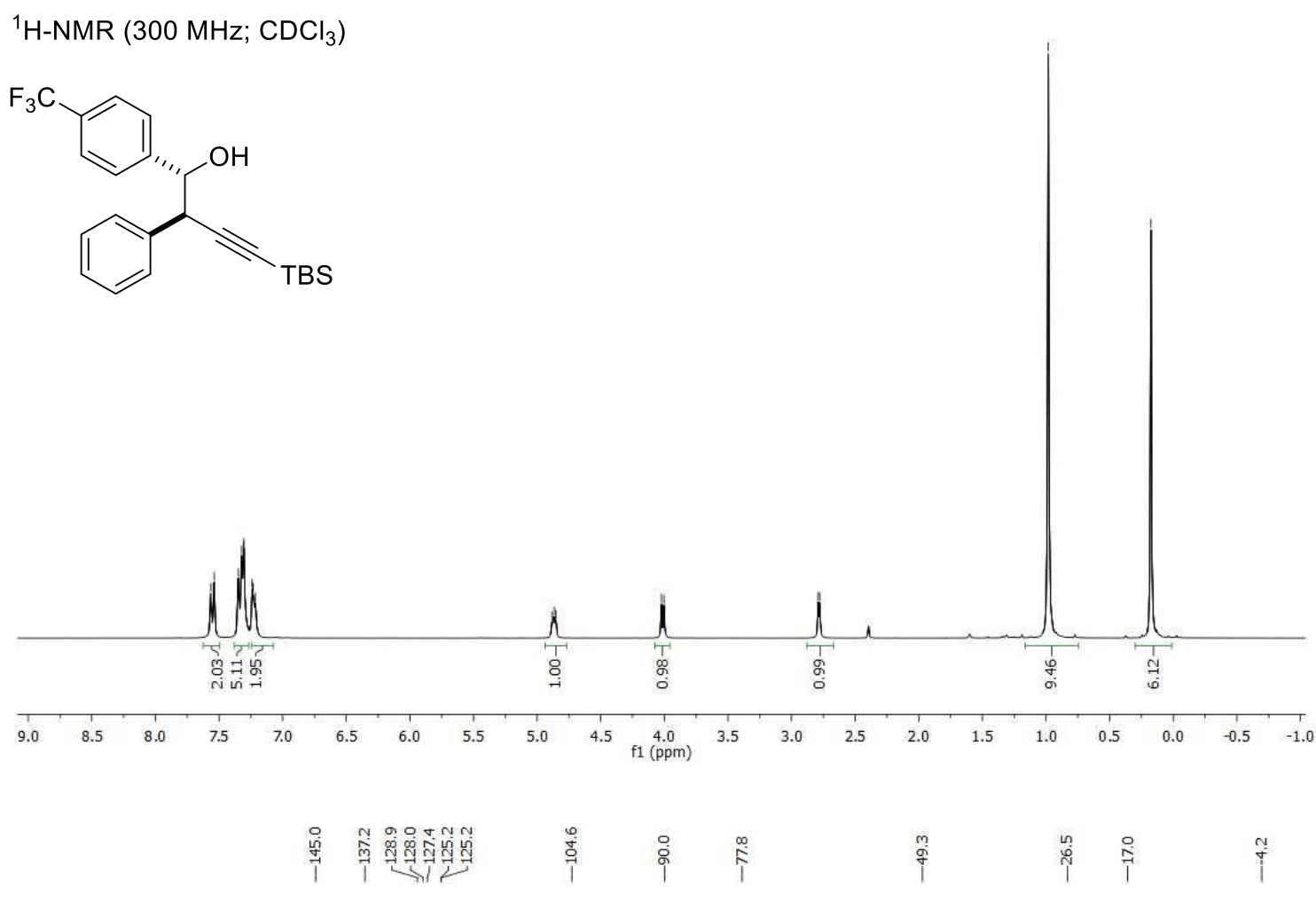

${ }^{13} \mathrm{C}-\mathrm{NMR}\left(75 \mathrm{MHz} ; \mathrm{CDCl}_{3}\right)$

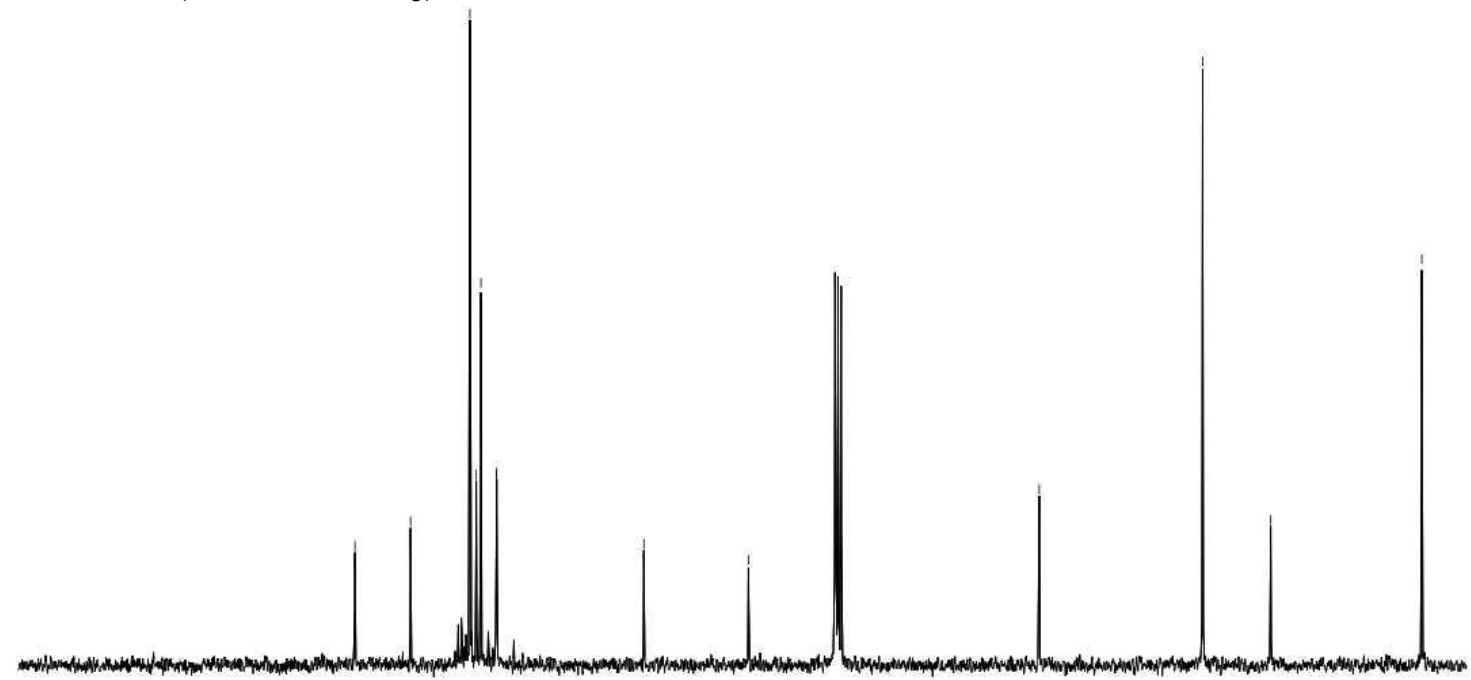

$\begin{array}{llllllllllllllllllllll}190 & 180 & 170 & 160 & 150 & 140 & 130 & 120 & 110 & 100 & \begin{array}{c}1 \\ \mathrm{fp} \\ (\mathrm{ppm})\end{array} & 80 & 70 & 60 & 50 & 40 & 30 & 20 & 10 & 0 & -10\end{array}$ 


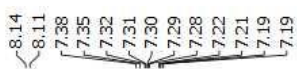

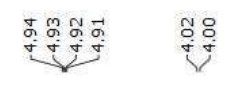

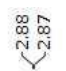

${ }^{1} \mathrm{H}-\mathrm{NMR}\left(300 \mathrm{MHz} ; \mathrm{CDCl}_{3}\right)$<smiles>C[Pb](C)(C)C#C[C@H](c1ccccc1)[C@H](O)c1ccc([N+](=O)[O-])cc1</smiles>

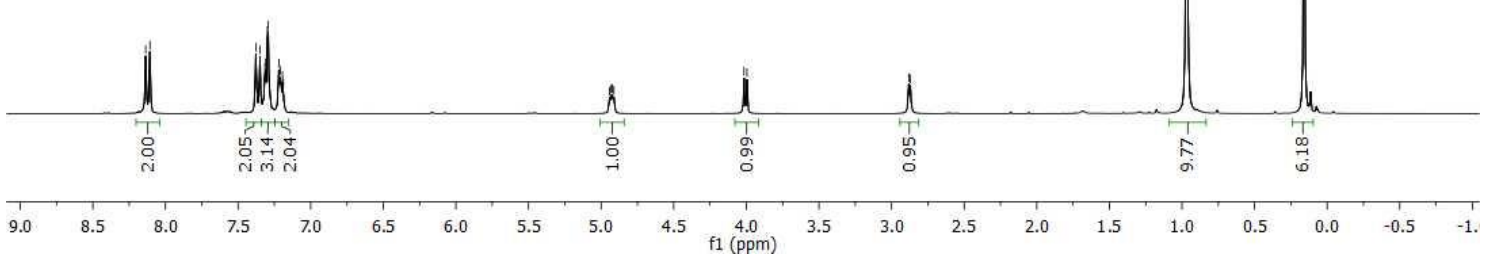

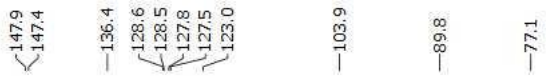

${ }^{13} \mathrm{C}-\mathrm{NMR}\left(75 \mathrm{MHz} ; \mathrm{CDCl}_{3}\right.$ )

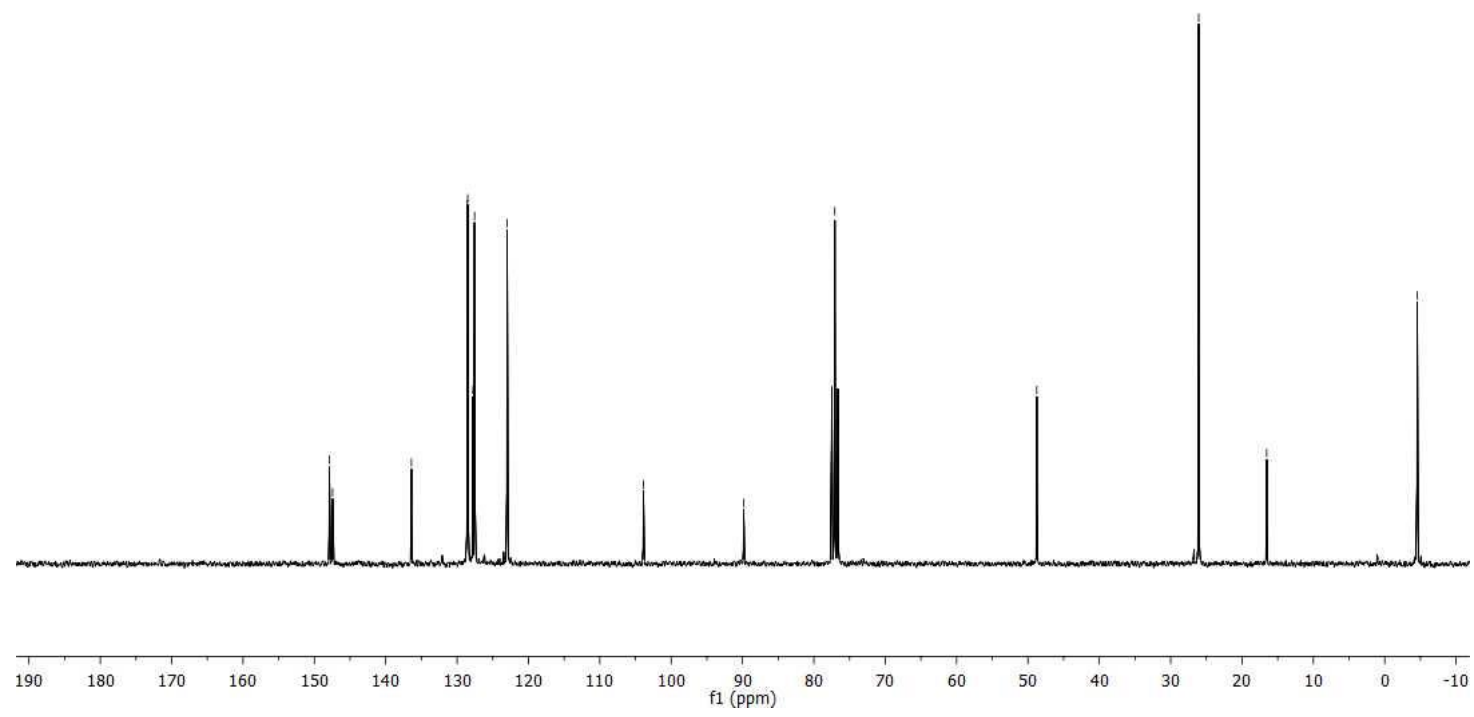




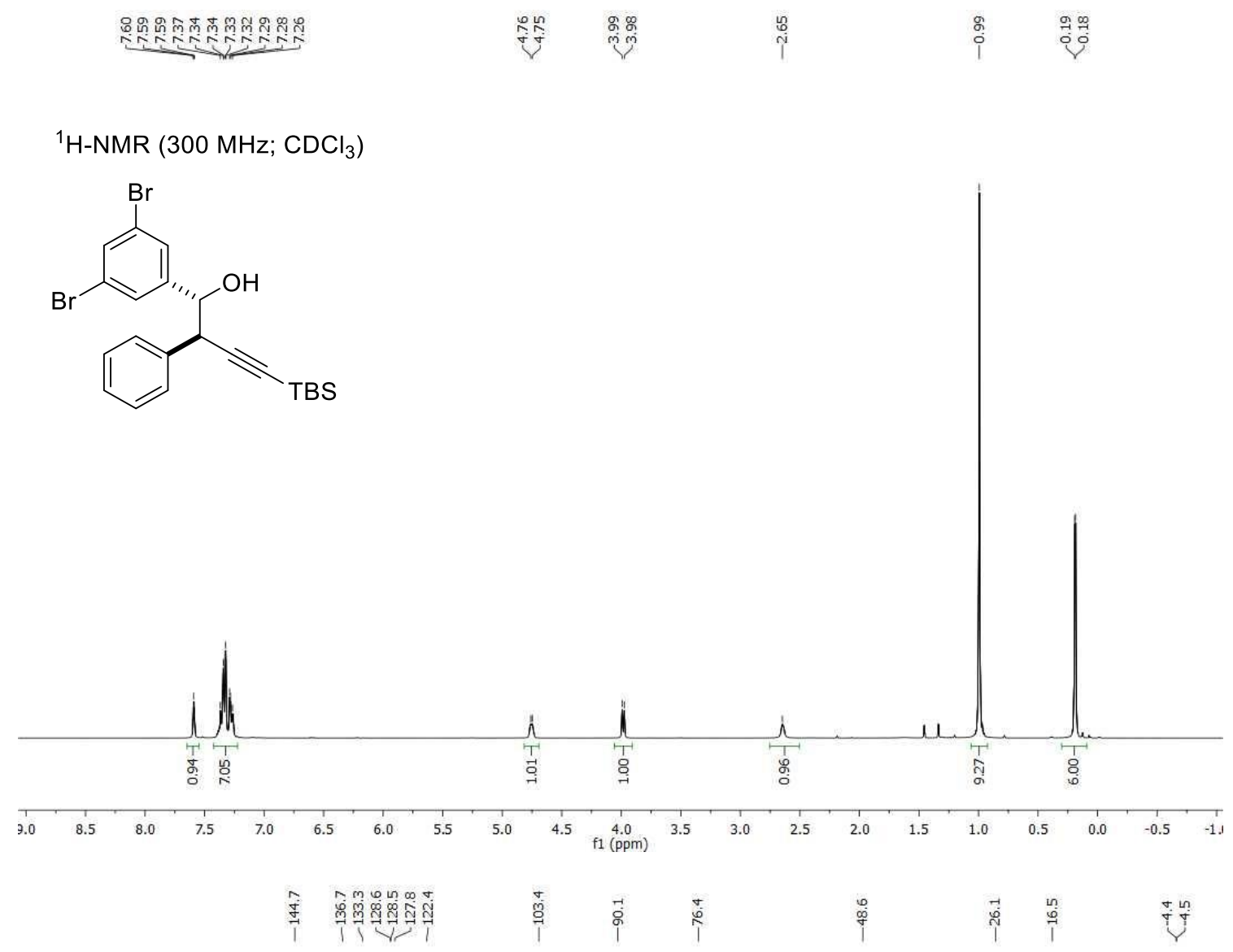

${ }^{13} \mathrm{C}-\mathrm{NMR}\left(75 \mathrm{MHz} ; \mathrm{CDCl}_{3}\right)$

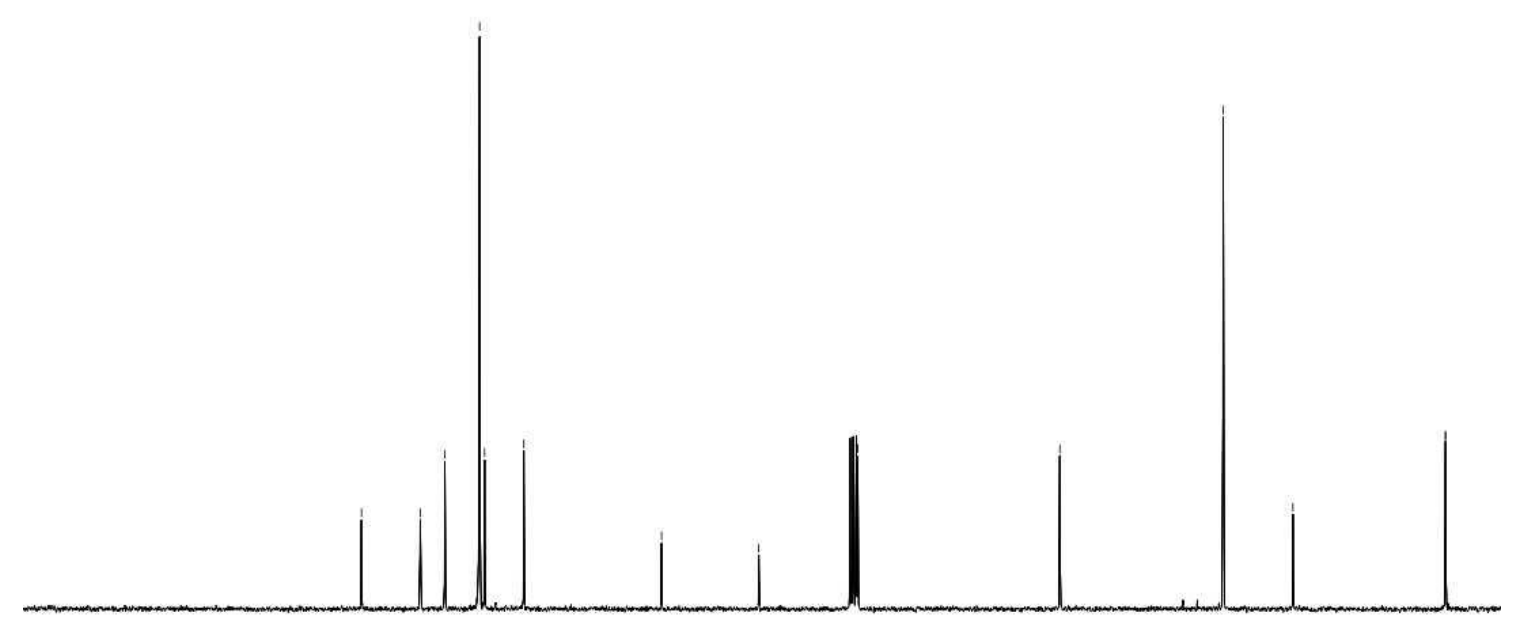

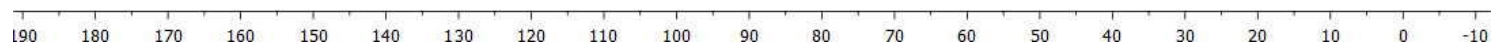




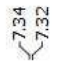

${ }^{1} \mathrm{H}-\mathrm{NMR}\left(300 \mathrm{MHz} ; \mathrm{CDCl}_{3}\right)$

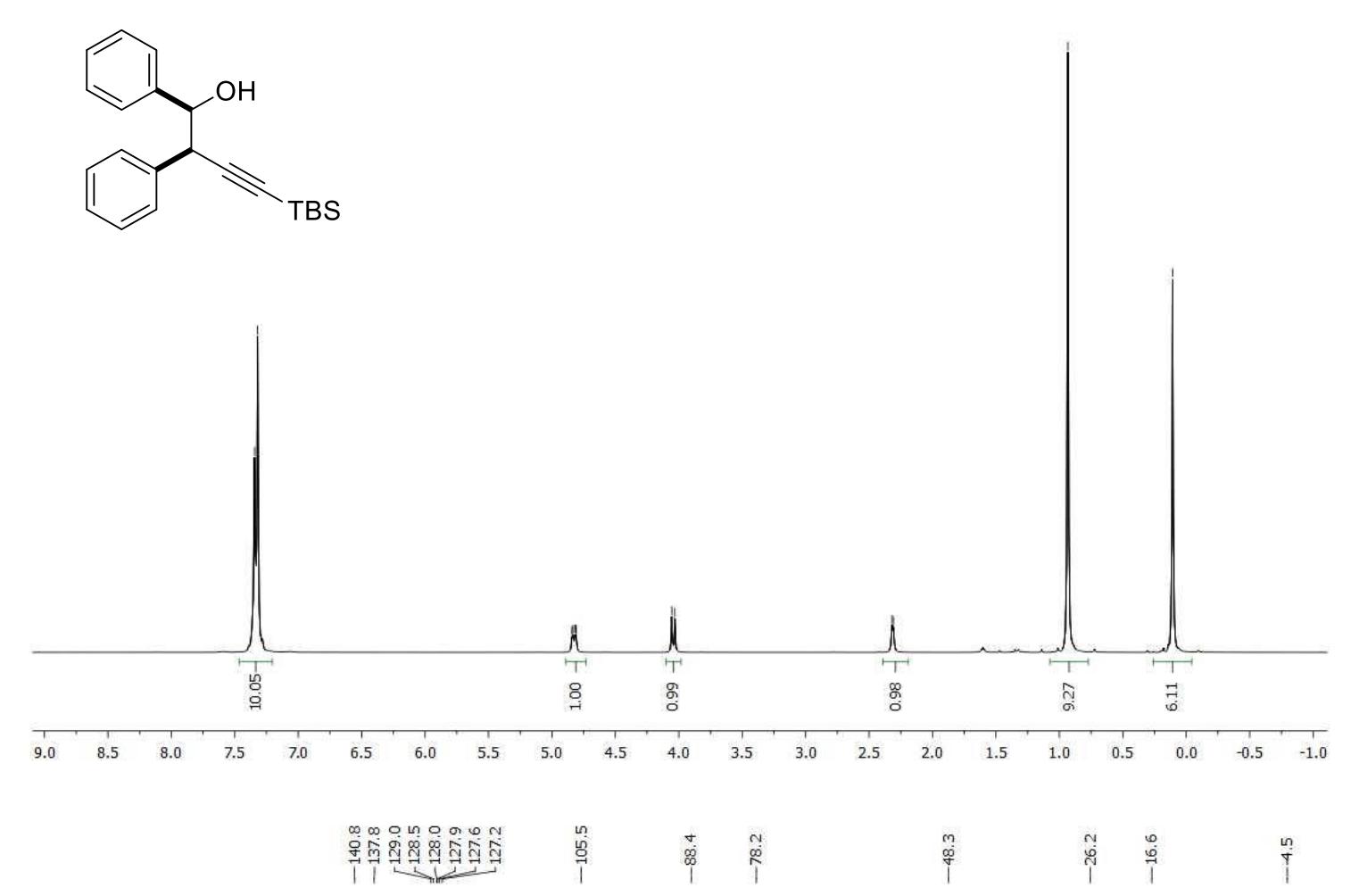

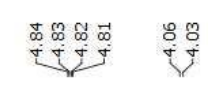

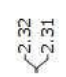

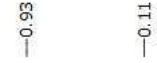

${ }^{13} \mathrm{C}-\mathrm{NMR}\left(75 \mathrm{MHz} ; \mathrm{CDCl}_{3}\right)$

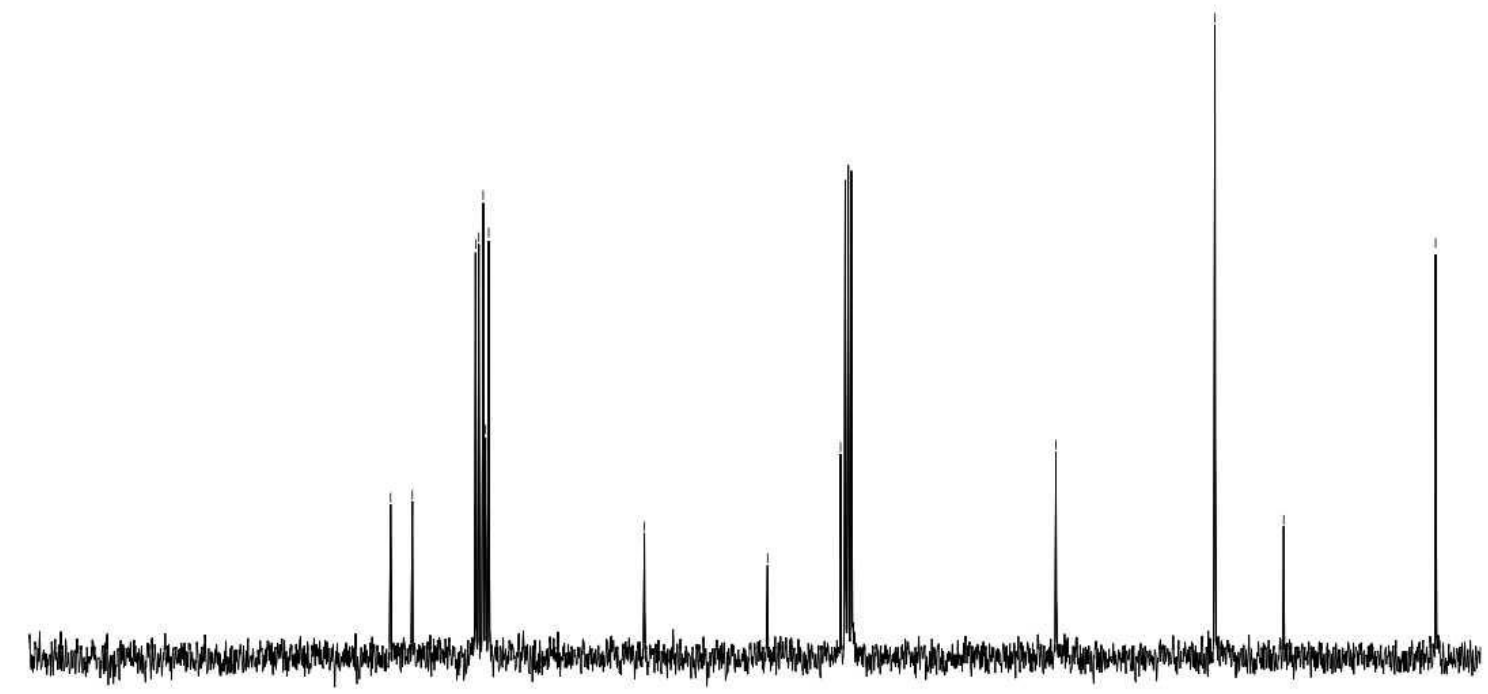

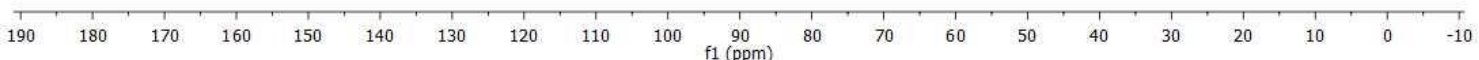


Syn-4-(tert-butyldimethylsilyl)-2-phenyl-1-(p-tolyl)but-3-yn-1-ol (syn-5b)

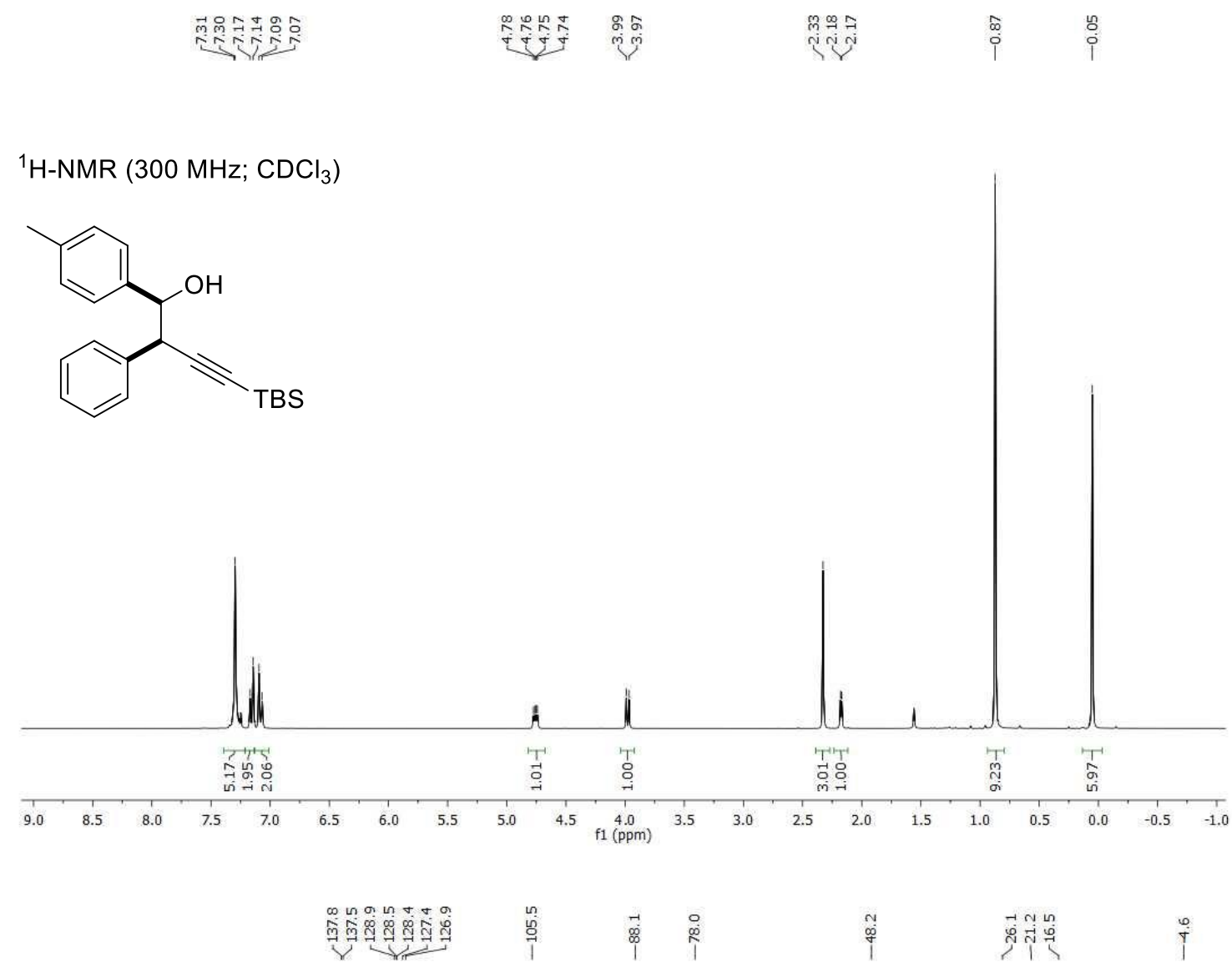

${ }^{13} \mathrm{C}-\mathrm{NMR}\left(75 \mathrm{MHz} ; \mathrm{CDCl}_{3}\right.$ )

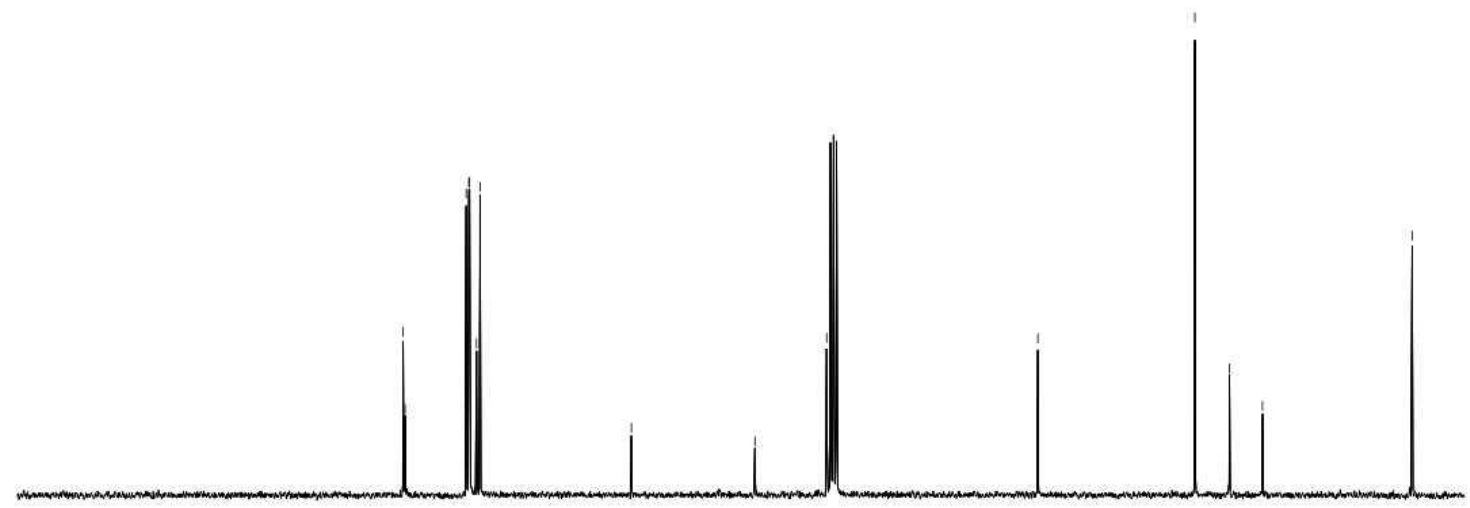




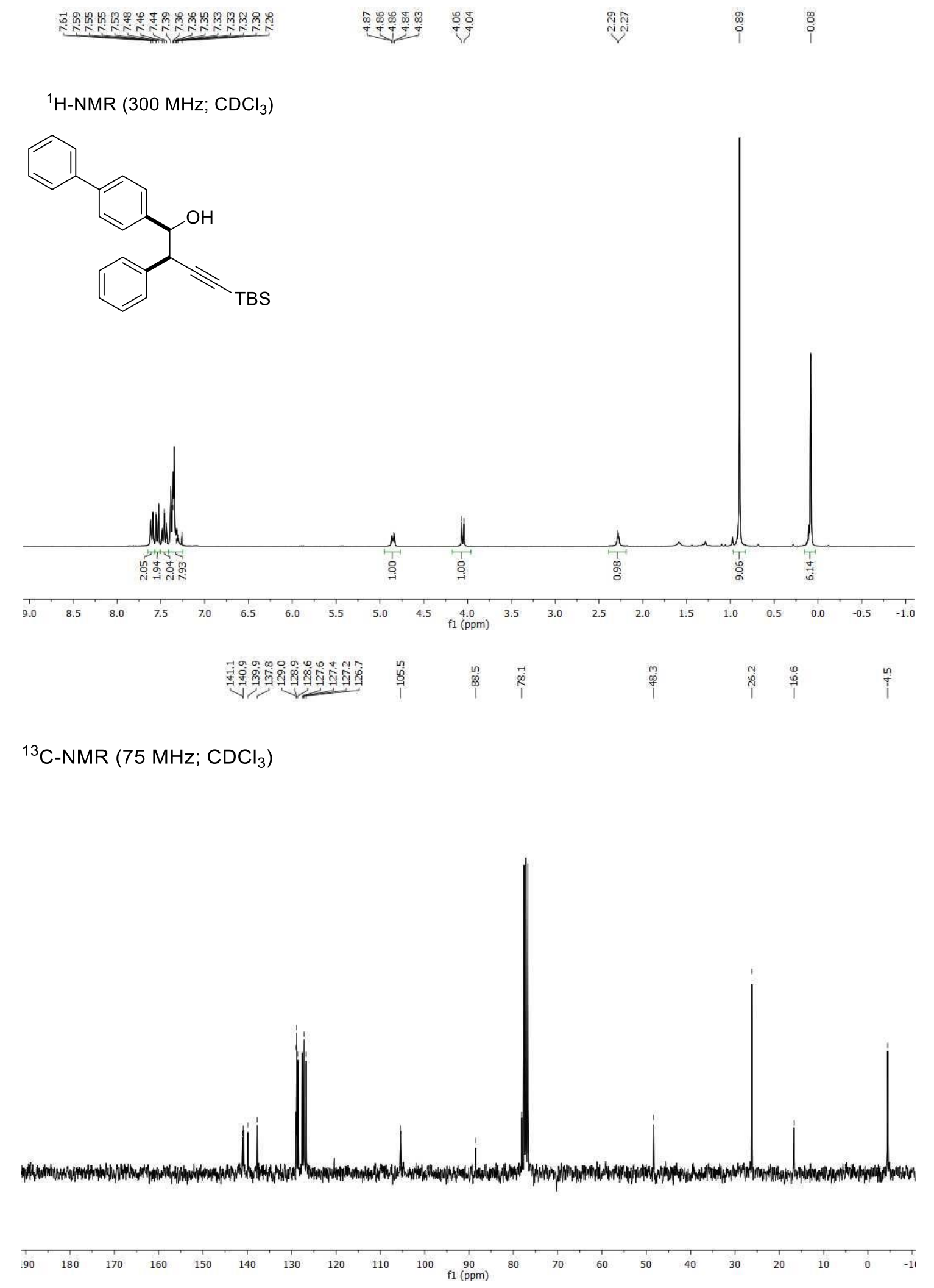




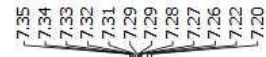
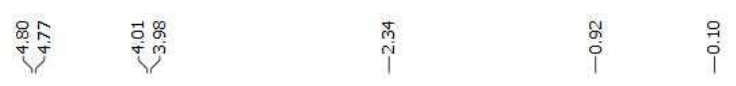

${ }^{1} \mathrm{H}-\mathrm{NMR}\left(300 \mathrm{MHz} ; \mathrm{CDCl}_{3}\right.$ )

$\mathrm{Cl}$<smiles>OC(C#C[181I])c1ccccc1</smiles>

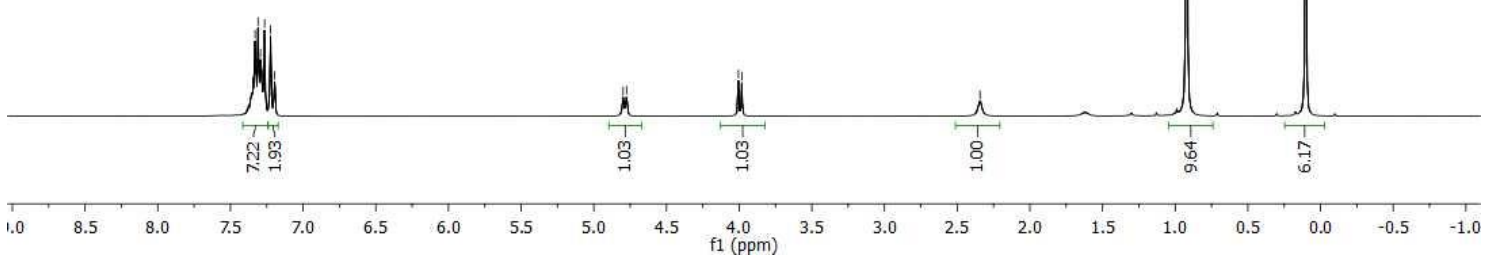

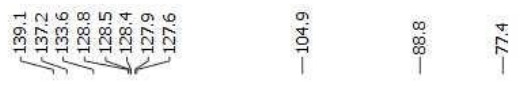

${ }^{13} \mathrm{C}-\mathrm{NMR}\left(75 \mathrm{MHz} ; \mathrm{CDCl}_{3}\right.$ )

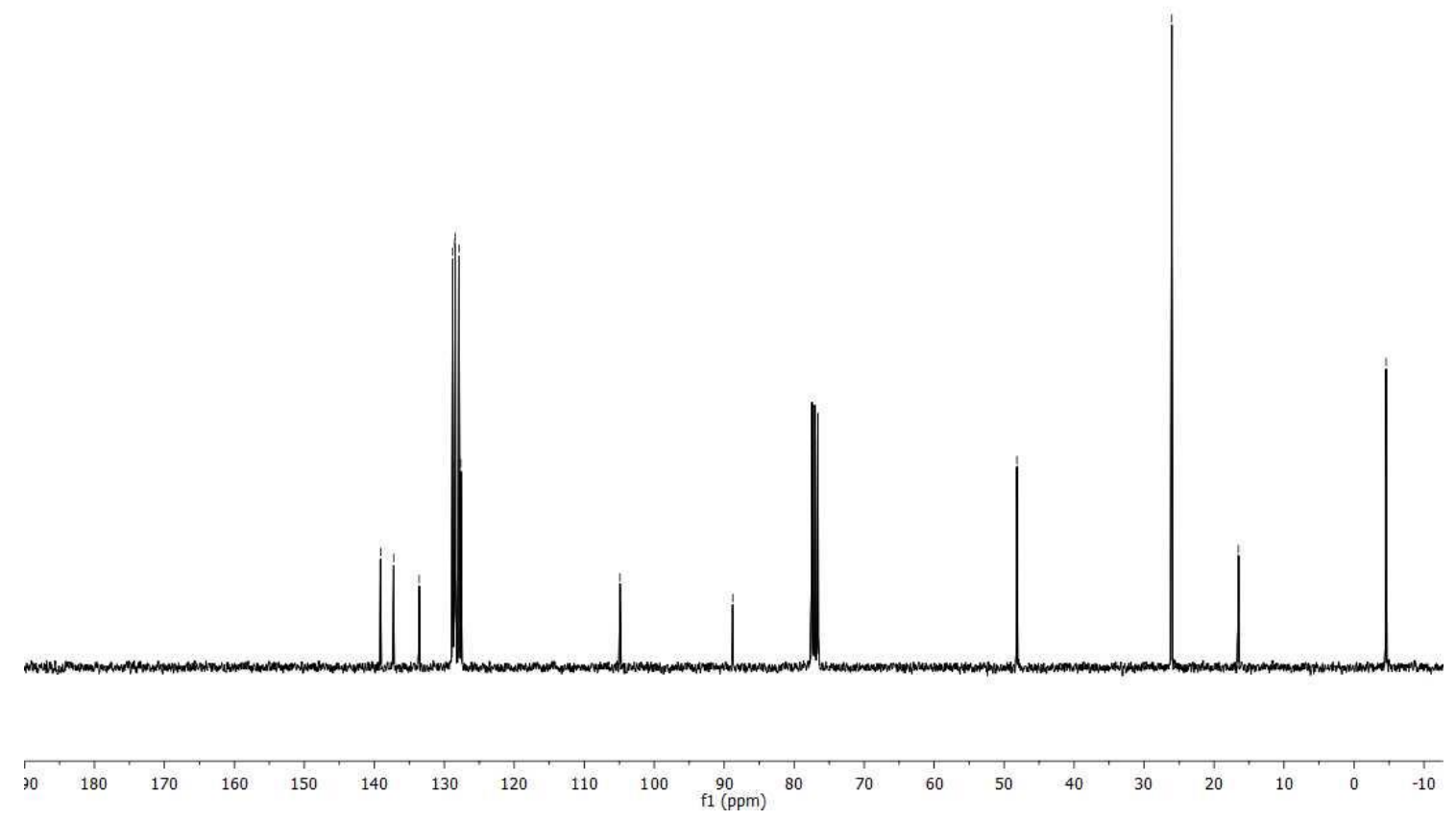


Syn-1-(4-Bromophenyl)-4-(tert-butyldimethylsilyl)-2-phenylbut-3-yn-1-ol (syn-5g)

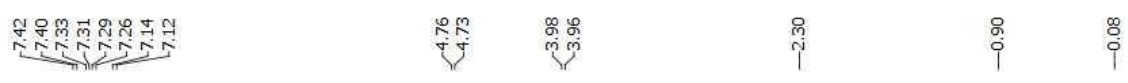

${ }^{1} \mathrm{H}-\mathrm{NMR}\left(300 \mathrm{MHz} ; \mathrm{CDCl}_{3}\right)$<smiles>CC(C)(C)C#CC(O)C(c1ccccc1)c1ccc(Br)cc1</smiles>
TBS
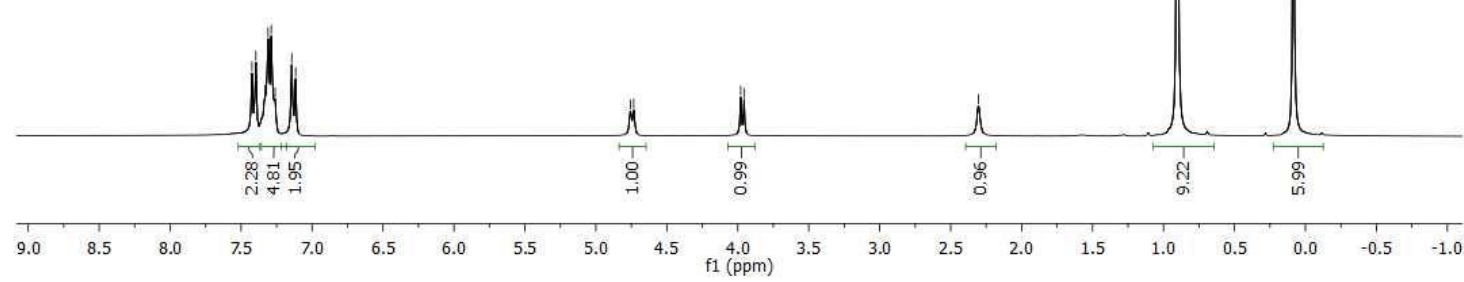

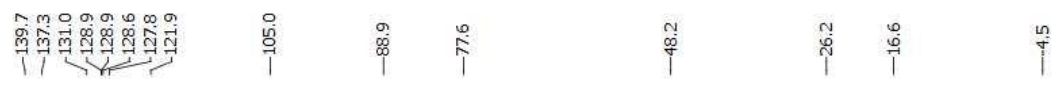

${ }^{13} \mathrm{C}-\mathrm{NMR}\left(75 \mathrm{MHz} ; \mathrm{CDCl}_{3}\right)$

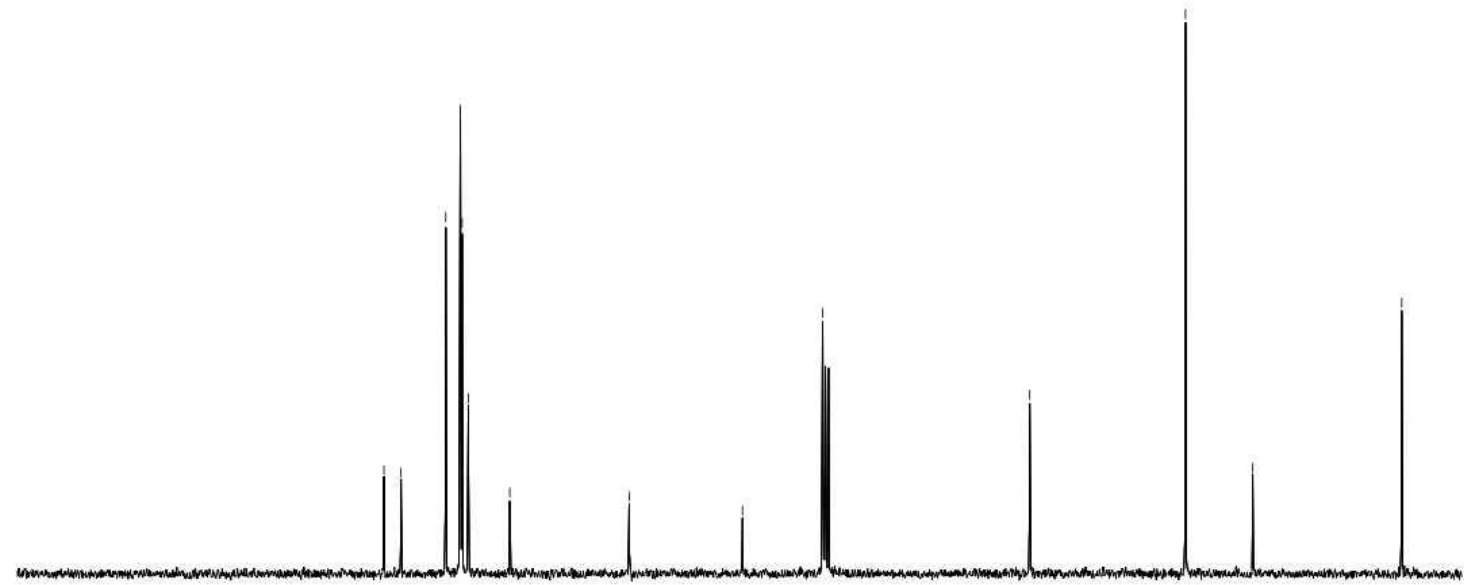

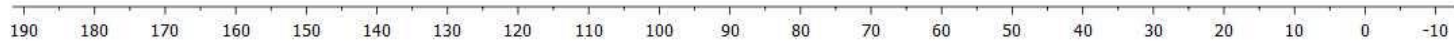




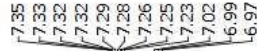

${ }^{1} \mathrm{H}-\mathrm{NMR}\left(300 \mathrm{MHz} ; \mathrm{CDCl}_{3}\right.$ )

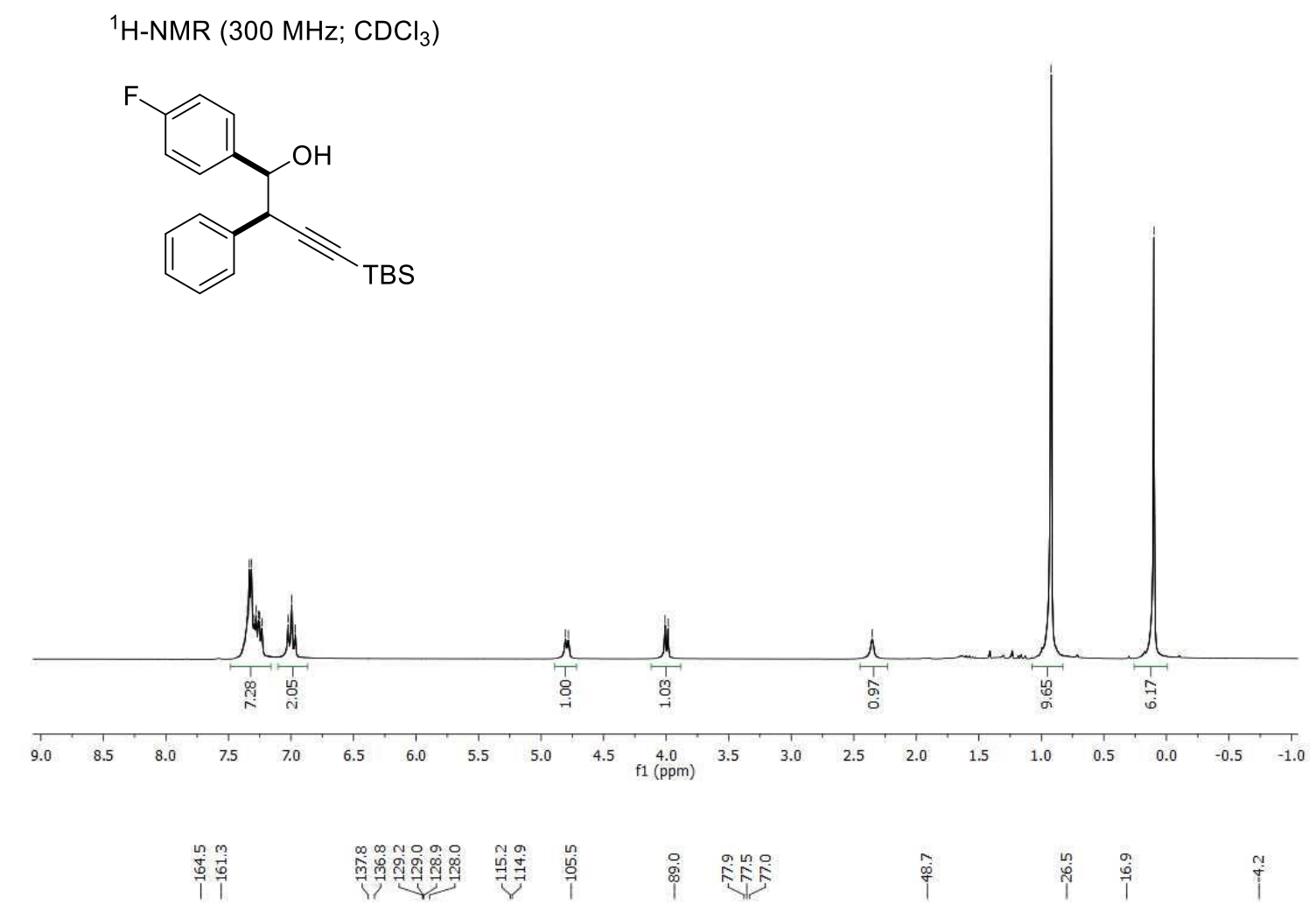

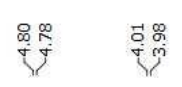

i̊n.

${ }^{13} \mathrm{C}-\mathrm{NMR}\left(75 \mathrm{MHz} ; \mathrm{CDCl}_{3}\right.$ )

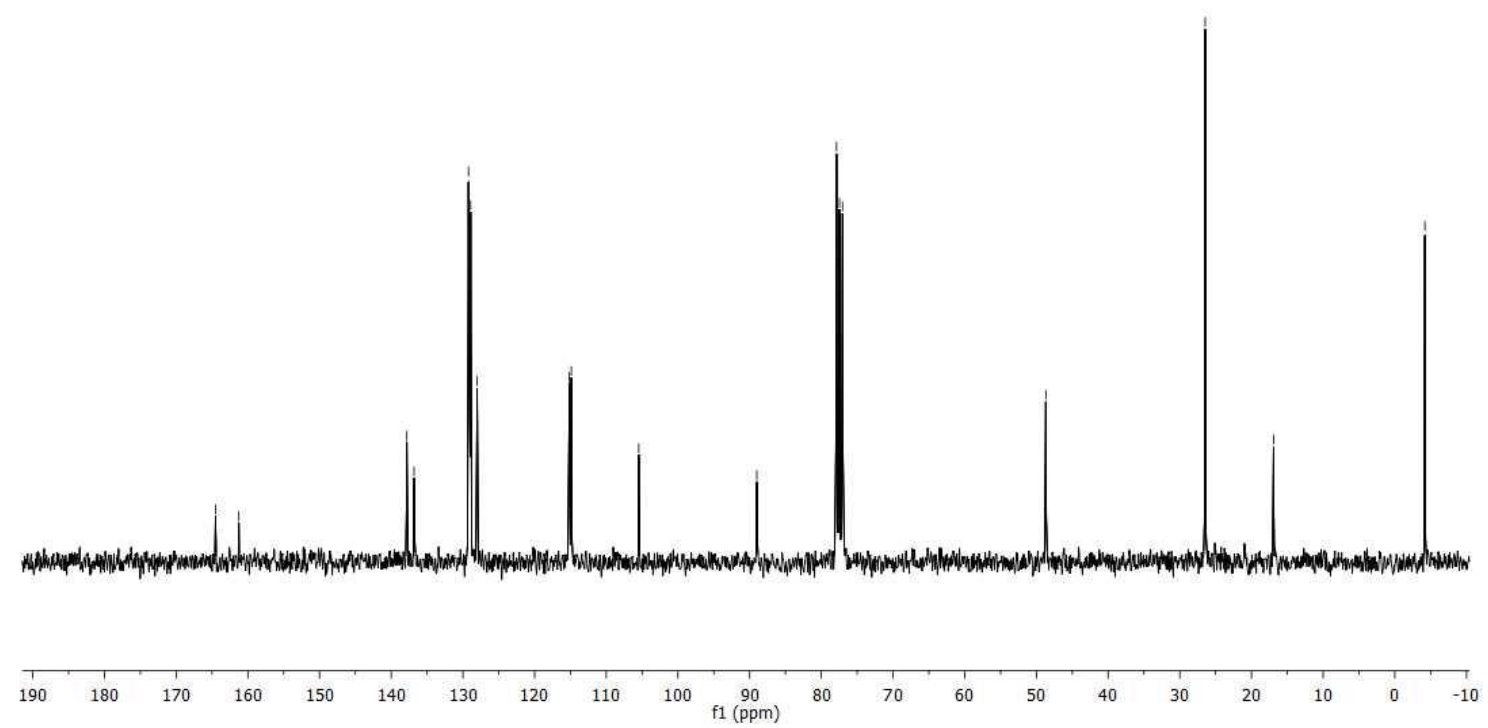


${ }^{1} \mathrm{H}-\mathrm{NMR}\left(300 \mathrm{MHz} ; \mathrm{CDCl}_{3}\right)$<smiles>CC(C)(C)C1=CC(c2ccccc2)C(c2ccccc2)O1</smiles>

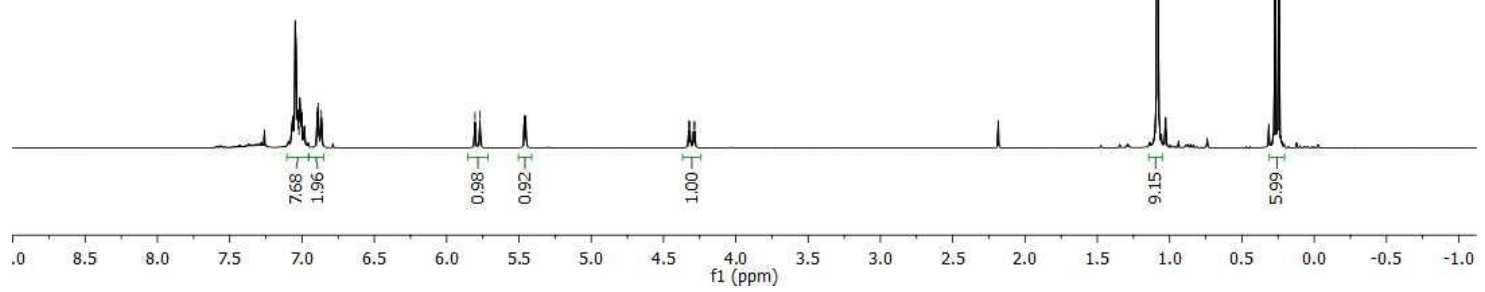

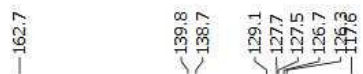

$\vec{\Phi}$

$\underset{\substack{1 \\ \uparrow}}{\substack{1 \\ 1}}$

${ }^{13} \mathrm{C}-\mathrm{NMR}\left(75 \mathrm{MHz} ; \mathrm{CDCl}_{3}\right)$

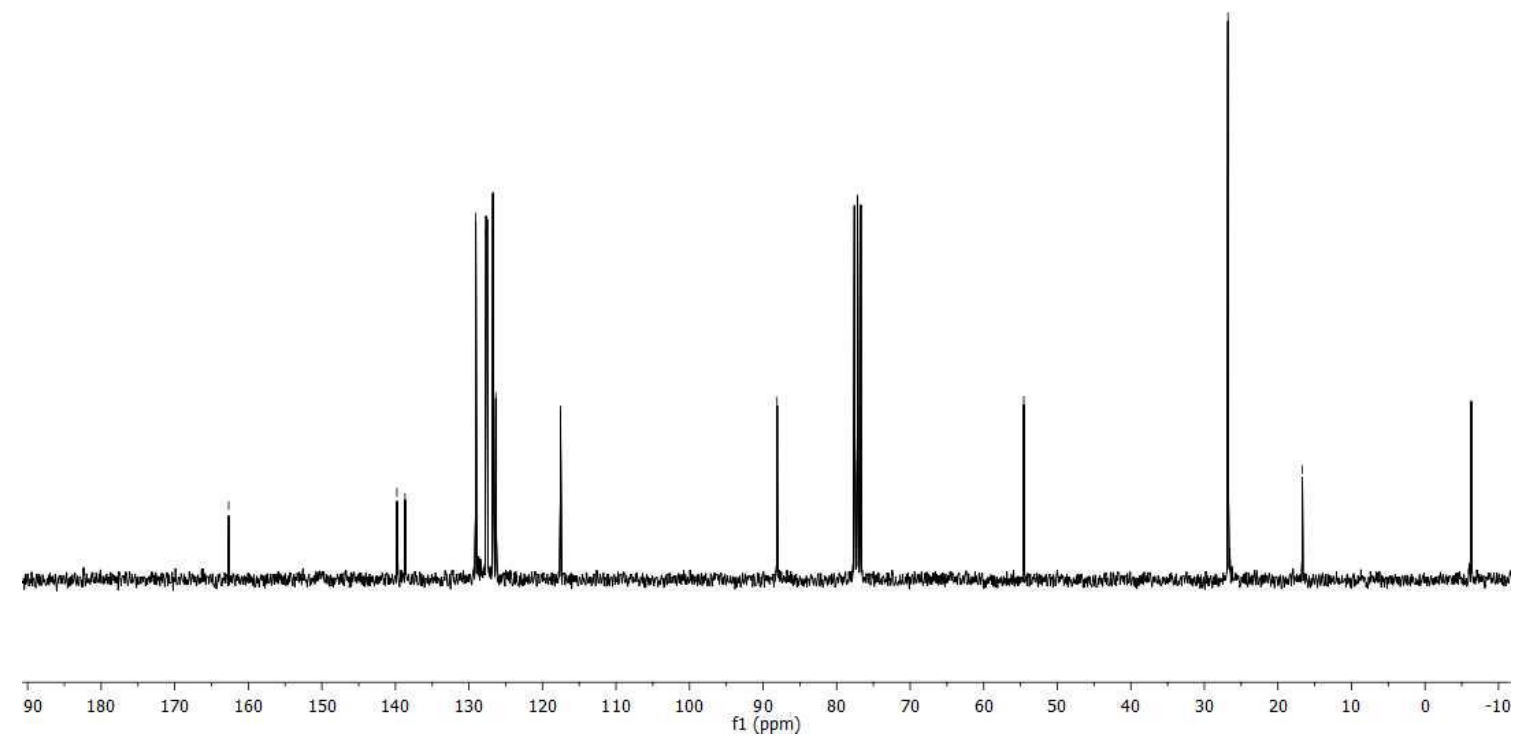


Cis-tert-butyldimethyl(4-phenyl-5-(p-tolyl)-4,5-dihydrofuran-2-yl)silane (3b)
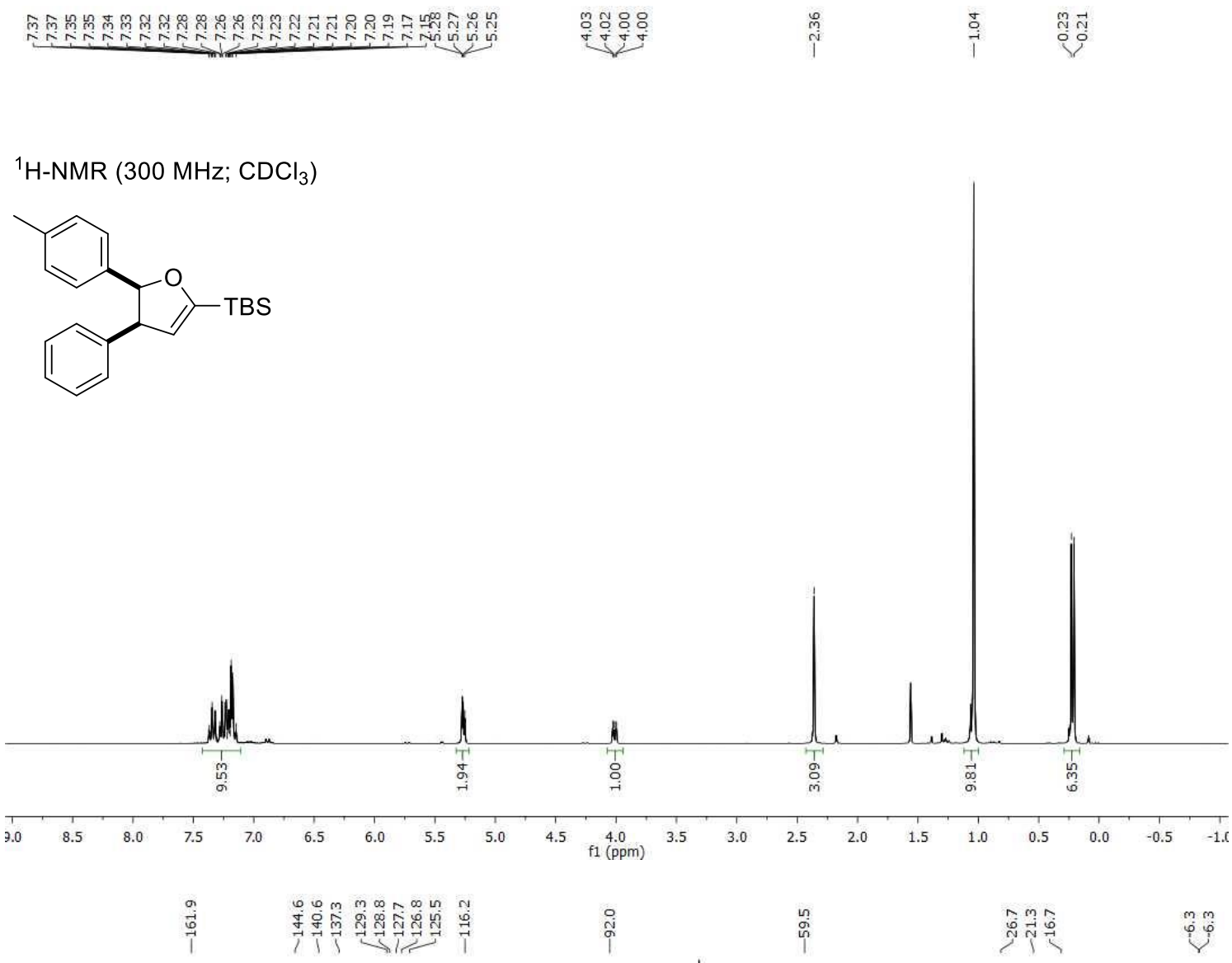

${ }^{13} \mathrm{C}-\mathrm{NMR}\left(75 \mathrm{MHz} ; \mathrm{CDCl}_{3}\right.$ )
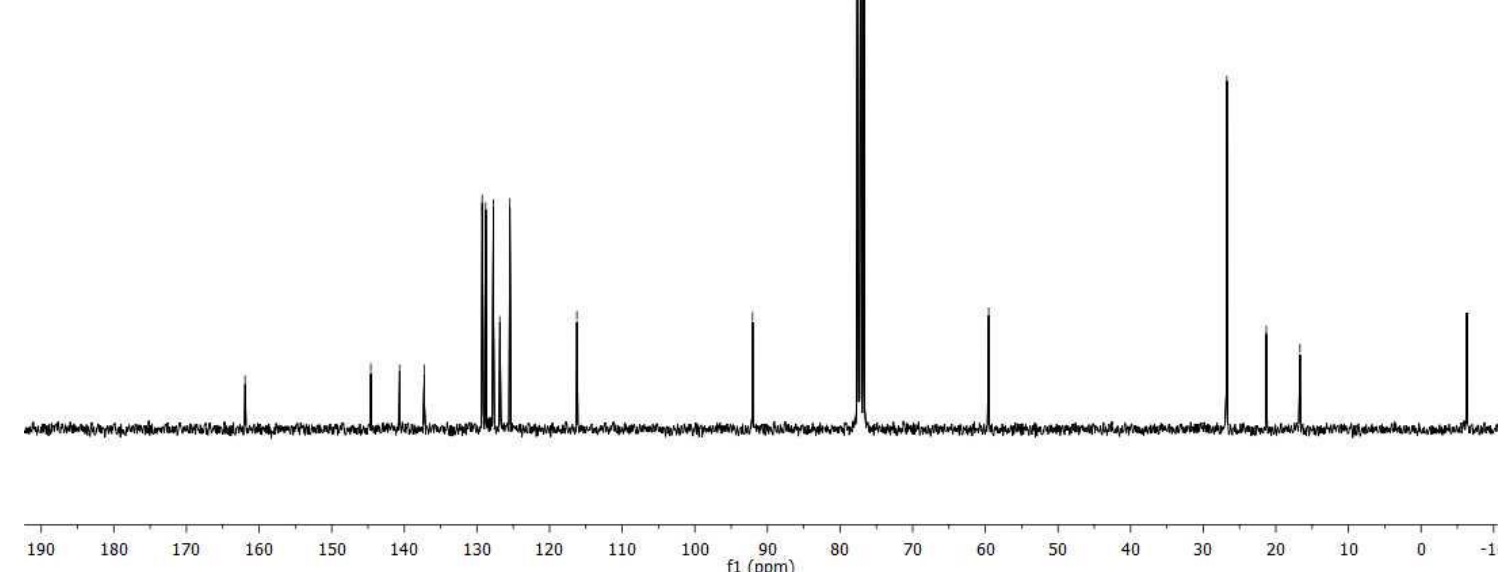
Cis-(5-([1,1'-biphenyl]-4-yl)-4-phenyl-4,5-dihydrofuran-2-yl)(tert-butyl)dimethylsilane (3d)

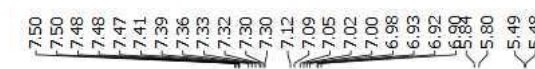

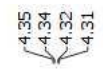

${ }^{1} \mathrm{H}-\mathrm{NMR}\left(300 \mathrm{MHz} ; \mathrm{CDCl}_{3}\right)$<smiles>CC(C)(C)C1=C[C@H](c2ccccc2)[C@H](c2ccc(-c3ccccc3)cc2)O1</smiles>

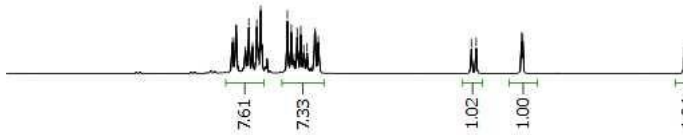

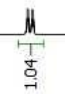

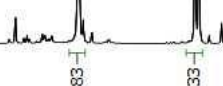

$\stackrel{T}{9.0}$

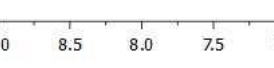

तิ

${ }^{13} \mathrm{C}-\mathrm{NMR}\left(75 \mathrm{MHz} ; \mathrm{CDCl}_{3}\right.$ )
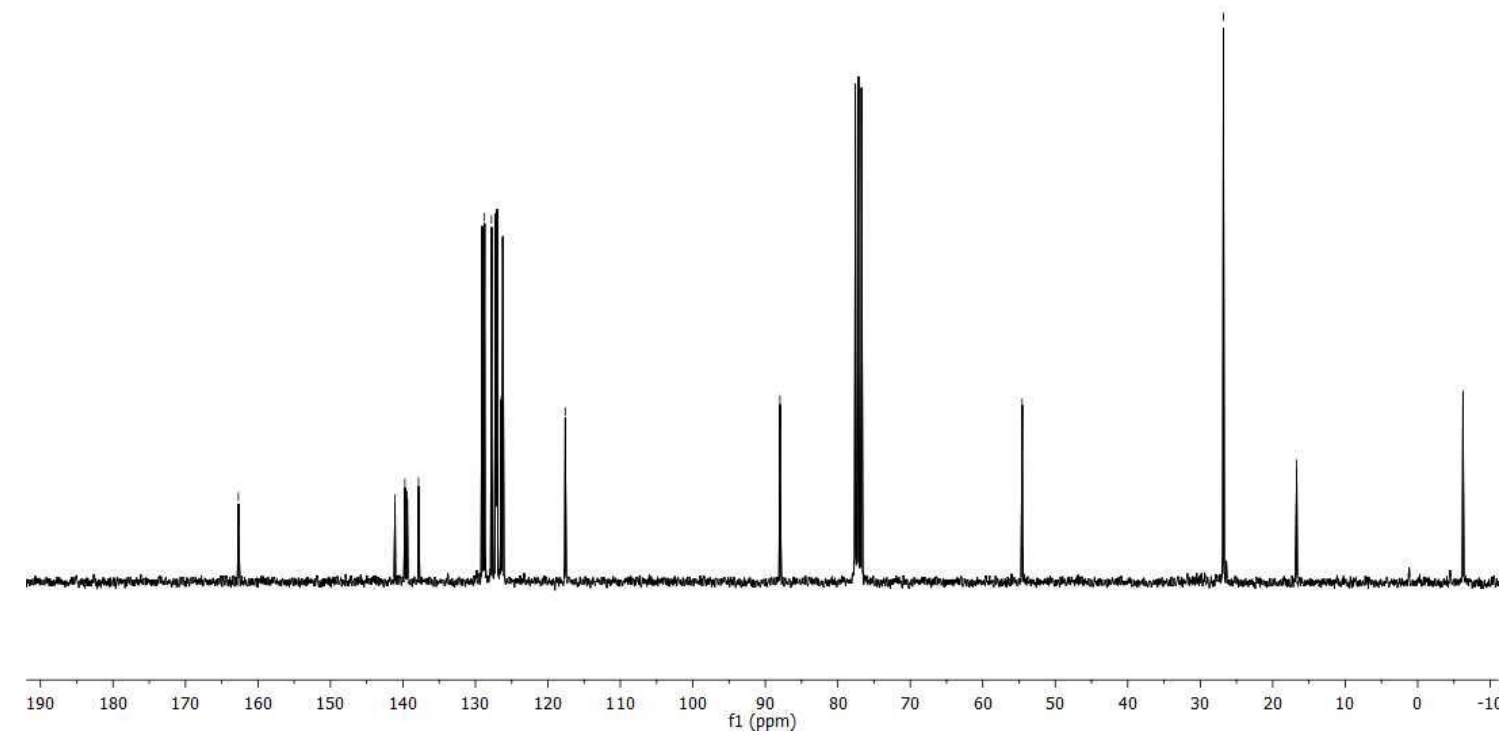


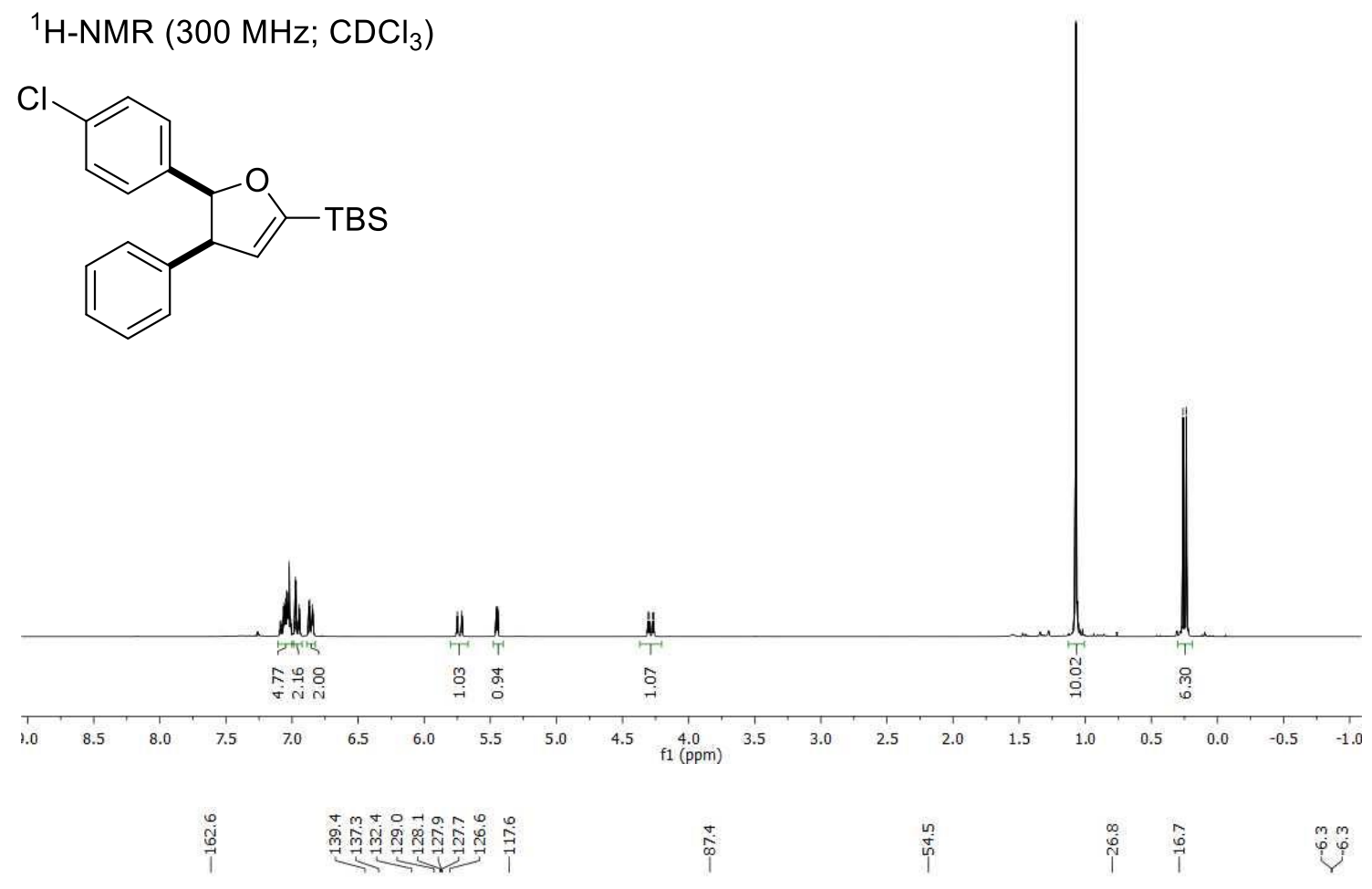

${ }^{13} \mathrm{C}-\mathrm{NMR}\left(75 \mathrm{MHz} ; \mathrm{CDCl}_{3}\right)$

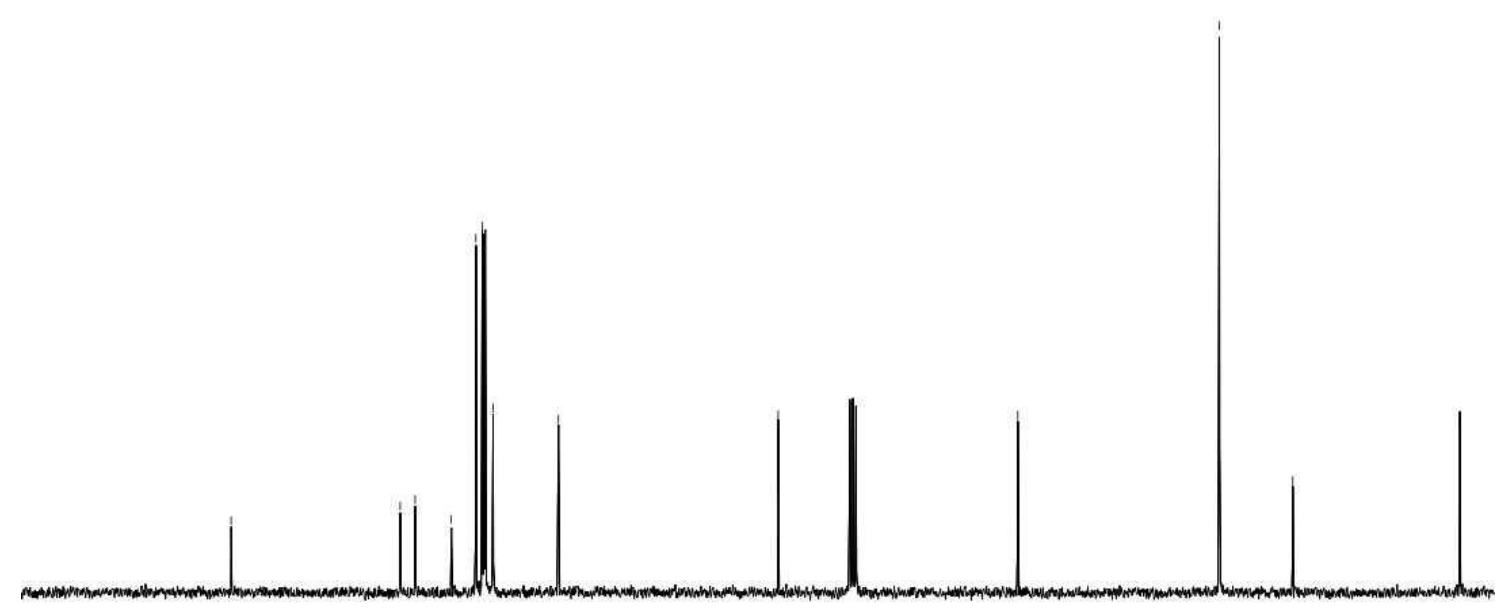

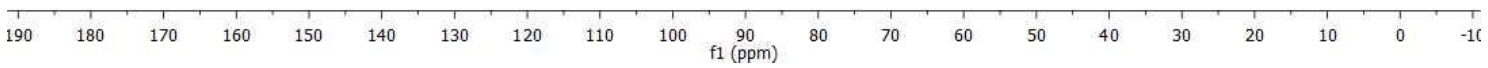


Cis-tert-butyldimethyl(5-(p-bromophenyl)-4-phenyl-4,5-dihydrofuran-2-yl)silane (3g)

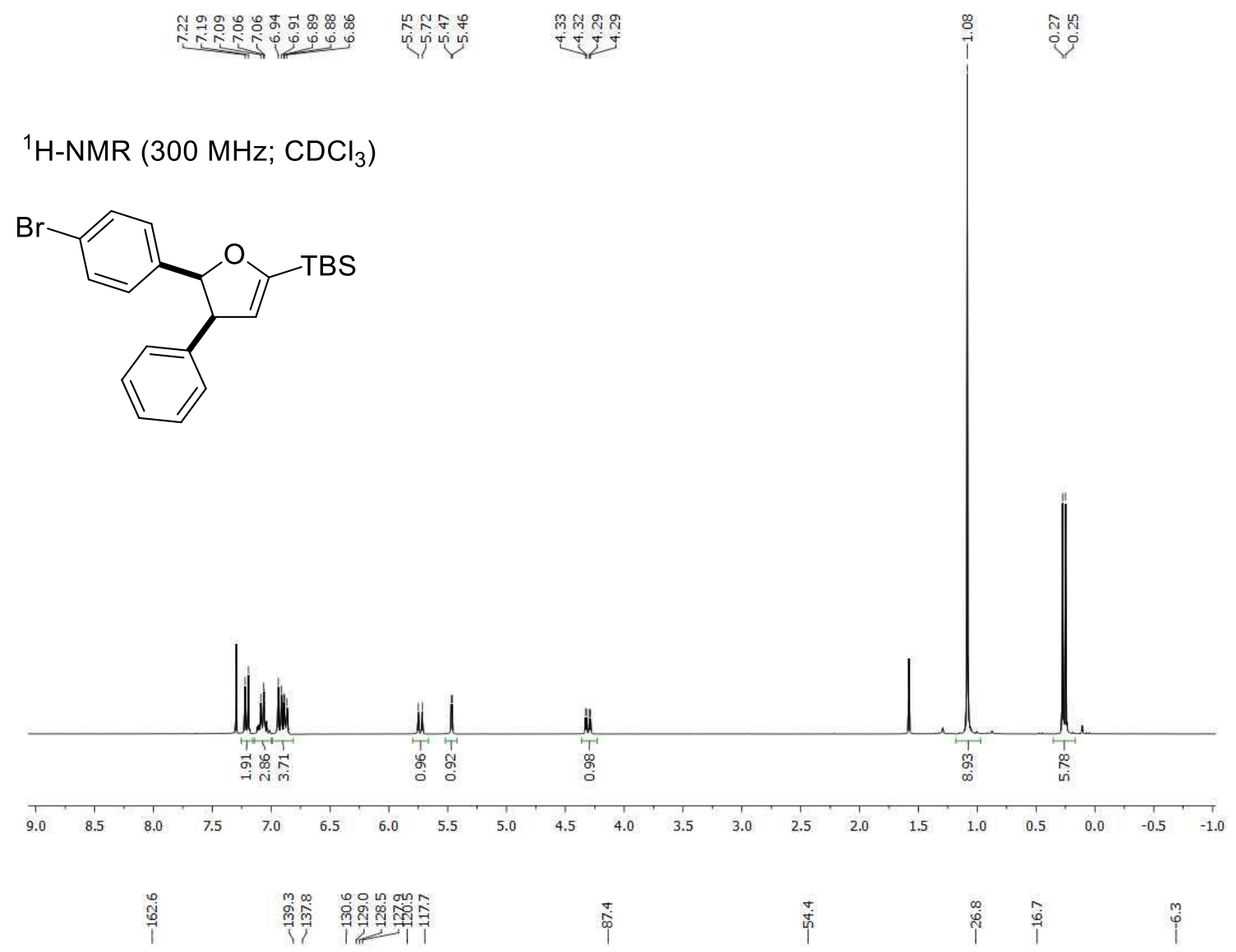

${ }^{13} \mathrm{C}-\mathrm{NMR}\left(75 \mathrm{MHz} ; \mathrm{CDCl}_{3}\right)$

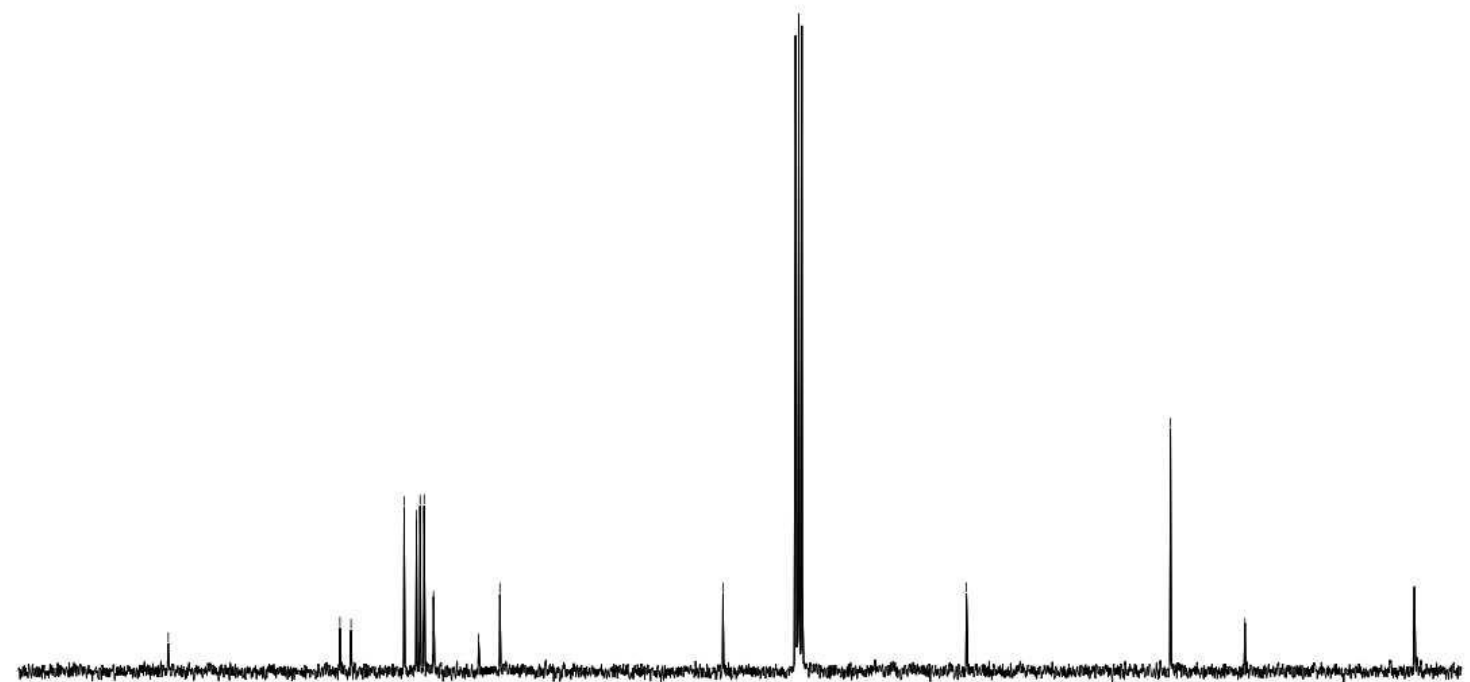

$\begin{array}{rllllllllllllllllllll}180 & 170 & 160 & 150 & 140 & 130 & 120 & 110 & 100 & 90 & 80 & 70 & 60 & 50 & 40 & 30 & 20 & 10 & 0 & -10\end{array}$ 


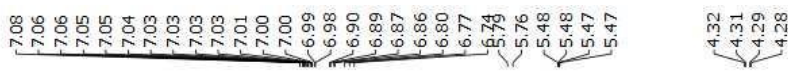

${ }^{1} \mathrm{H}-\mathrm{NMR}\left(300 \mathrm{MHz} ; \mathrm{CDCl}_{3}\right)$<smiles>CC(C)(C)C1=CC(c2ccccc2)C(c2ccc(F)cc2)O1</smiles>

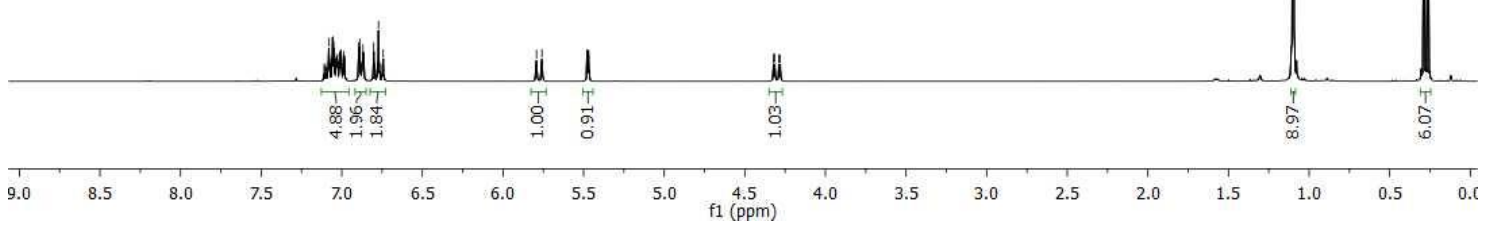

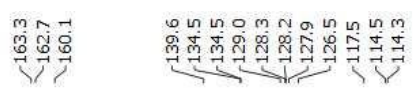

$\stackrel{\substack{0 \\ \infty}}{1}$

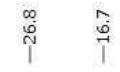

${ }^{13} \mathrm{C}-\mathrm{NMR}\left(75 \mathrm{MHz} ; \mathrm{CDCl}_{3}\right)$

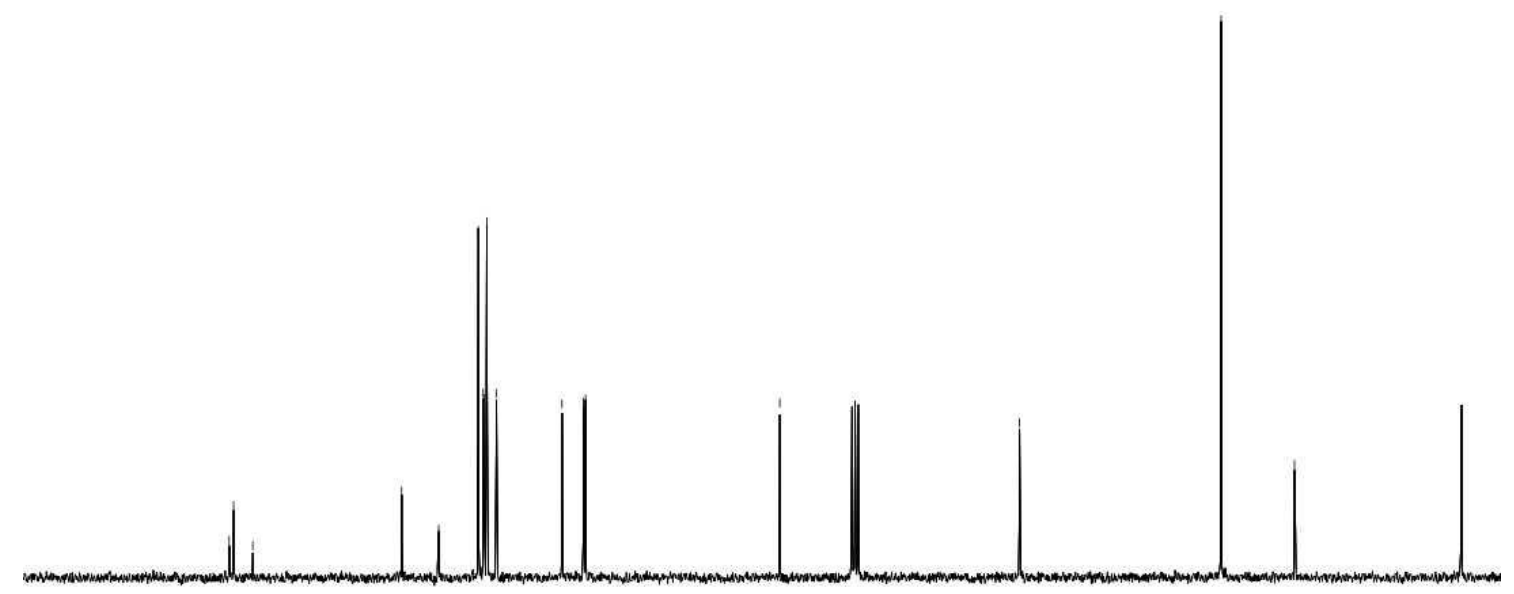

$\begin{array}{lllllllllllllllllll}1 & 1 & 1 & 1 & 1 & 1 & 1 & 1 & 1 & 1 & 1 & 1 & 1 & 1 & 1 & 1 & 1 & 1 & 1 \\ 1\end{array}$ 